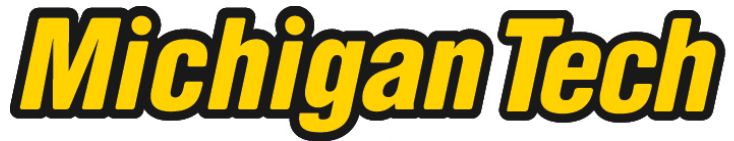 \\ Michigan Technological University Create the Future Digital Commons @ Michigan Tech
}

\section{Algorithms for spacecraft formation flying navigation based on wireless positioning system measurements}

Shu Ting Goh

Michigan Technological University

Follow this and additional works at: https://digitalcommons.mtu.edu/etds

Part of the Mechanical Engineering Commons

Copyright 2012 Shu Ting Goh

\section{Recommended Citation}

Goh, Shu Ting, "Algorithms for spacecraft formation flying navigation based on wireless positioning system measurements", Dissertation, Michigan Technological University, 2012.

https://doi.org/10.37099/mtu.dc.etds/365

Follow this and additional works at: https://digitalcommons.mtu.edu/etds

Part of the Mechanical Engineering Commons 


\title{
ALGORITHMS FOR SPACECRAFT FORMATION FLYING NAVIGATION BASED ON WIRELESS POSITIONING SYSTEM MEASUREMENTS
}

$$
\text { By: }
$$

Shu Ting Goh

\author{
A DISSERTATION \\ Submitted in partial fullfilment of the requirements for the degree of \\ DOCTOR OF PHILOSOPHY \\ (Mechanical Engineering-Engineering Mechanics)
}

MICHIGAN TECHNOLOGICAL UNIVERSITY

2012

2012 Copyright @Shu Ting Goh 

This dissertation, "Algorithms For Spacecraft Formation Flying Navigation Based on Wireless Positioning System Measurements," is hereby approved in partial fulfillment of the requirements for the degree of DOCTOR OF PHILOSOPHY in the field of Mechanical Engineering - Engineering of Mechanics.

Department of Mechanical Engineering - Engineering of Mechanics

Signatures:

Dissertation Co-Advisor

Ossama Abdelkhalik

Dissertation Co-Advisor

Seyed A. (Reza) Zekavat

Committee member

Chris E. Passerello

Committee member

Bo Chen

Department Chair

William W. Predebon

Date 



\section{Contents}

List of Figures . . . . . . . . . . . . . . . . xiv

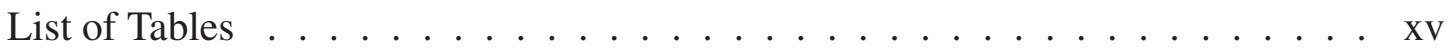

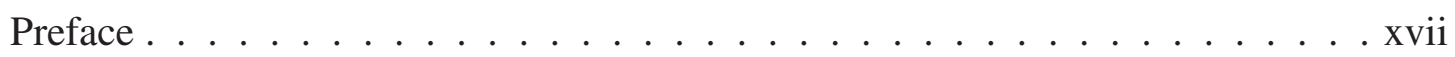

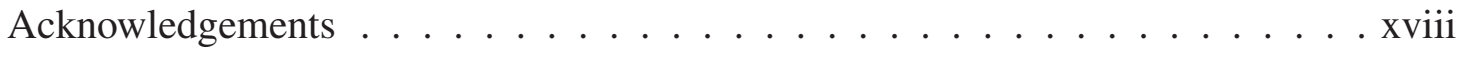

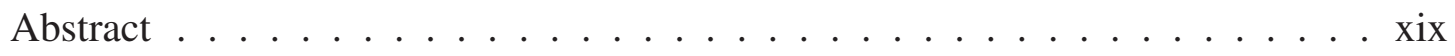

1 Introduction $\ldots \ldots \ldots \ldots \ldots \ldots \ldots \ldots \ldots \ldots \ldots \ldots$

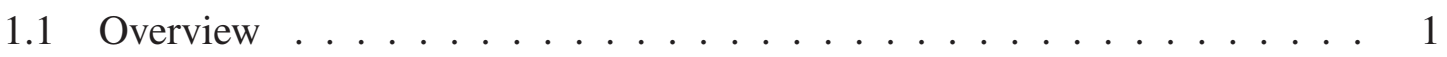

1.2 Optimal State Estimation and Navigation . . . . . . . . . . 3

1.3 Navigation/Localization Sensors . . . . . . . . . . . . 5

1.4 Objectives of this Dissertation $\ldots \ldots \ldots \ldots$

1.5 Organization of this Dissertation . . . . . . . . . . 7

2 Spacecraft Formation Orbit Estimation using WLPS-based Localization . . 9

2.1 Introduction . . . . . . . . . . . . . . 10

2.2 Wireless Local Positioning System (WLPS) . . . . . . . . . . . . 13

2.3 System Model . . . . . . . . . . . . . . . . . . . 14

2.3.1 State Model . . . . . . . . . . . . . . . . 14

2.3.2 Measurements Model . . . . . . . . . . . . . 15 
2.3.3 Time Delay Modeling . . . . . . . . . . . . . . . . 17

2.3.3.1 Sensitivity matrix for TOA with Time Delay Model _. . 21

2.3.3.2 Sensitivity matrix for DOA with Time Delay Model . . . 22

2.3.4 GPS Measurements Model . . . . . . . . . . . . . . . . . 23

2.4 Extended Kalman Filter Implementation . . . . . . . . . . . . . 24

2.5 Simulation Results and Discussions $\ldots \ldots \ldots \ldots$

2.5.1 One DOA measurement . . . . . . . . . . . . . . . . . 29

2.5.2 GPS and WLPS versus GPS standalone system . . . . . . . 30

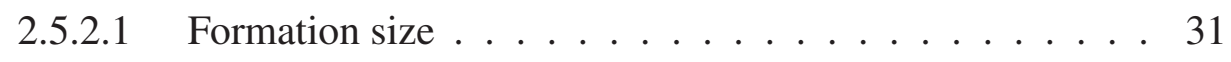

2.5.2.2 Measurement noise level . . . . . . . . . . . . 32

2.5.2.3 Number of spacecraft in formation $\ldots \ldots . . . . .33$

\section{Implementation of a Differential Geometric Filter For Spacecraft Formation}

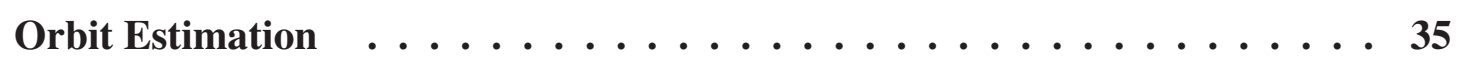

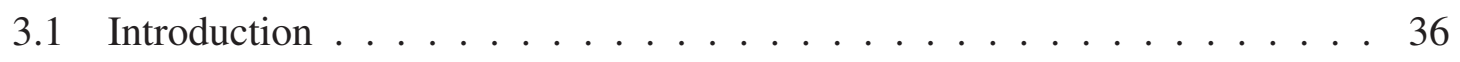

3.2 Measurement Model . . . . . . . . . . . . . . . . 38

3.2.1 WLPS Measurements Model . . . . . . . . . . . . . . 39

3.2.2 Radar System Model . . . . . . . . . . . . . . . . . . . . . 39

3.2.3 Measurement Models for Simulation . . . . . . . . . . . . 40

3.3 Differential Geometric Filter . . . . . . . . . . . . . 41

3.3.1 Linear Filtering Gain $\ldots \ldots \ldots$. . . . . . . . . . 42

3.3.2 Pole Placement Method . . . . . . . . . . . . . . . . . 44

3.3.3 Scenario One - Relative Position Estimation with WLPS-only measurement. . . . . . . . . . . . . . . 45

3.3.4 Scenario Two - Relative Position Estimation with WLPS and Radar measurement. . . . . . . . . . . . . . 48 
3.4 Implementation of Extended Kalman Filter . . . . . . . . . . . . . . . . 49

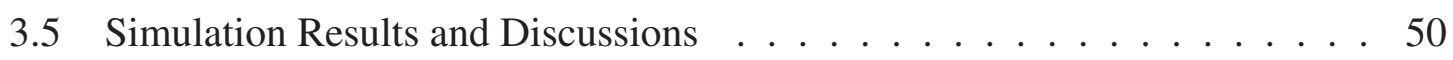

3.5.1 Observability analysis ................... 52

3.5 .2 Scenario One ....................... 52

3.5.3 Discussion - Scenario One . . . . . . . . . . . . . . 54

3.5.4 Scenario Two . . . . . . . . . . . . . . 55

3.5.4.1 Linear Filtering Gain . . . . . . . . . . . 56

3.5.4.2 Pole Placement Method . . . . . . . . . . . . 57

3.5.5 Complexity Analysis . . . . . . . . . . . . . 58

4 Constraint Estimation of Spacecraft Positions _............. 61

4.1 Introduction . . . . . . . . . . . . . . . . 62

4.2 Constrained Kalman Filter . . . . . . . . . . . . . . . . 65

4.3 Spacecraft Position and Velocity at Perigee and Apogee . . . . . . . . . . 69

4.3 .1 Circular Orbit . . . . . . . . . . . . . 70

4.3.2 Elliptic orbit with well-known $\Delta t_{p}$ and $\Delta t_{a} \ldots \ldots \ldots$

4.3.3 Elliptic orbit with unknown $\Delta t_{p}$ and $\Delta t_{a} \ldots \ldots \ldots 71$

4.4 Simulation and Discussion . . . . . . . . . . . . . 72

4.4.1 Absolute Position Estimation with Radar Tracking . . . . . . . . . 74

4.4.2 Scenario two - formation flying . . . . . . . . . 76

4.4.2.1 Formation Flying in a circular orbit . . . . . . . . . 76

4.4.2.2 SFF in eccentric orbit - known perigee/apogee times . . . 77

4.4.2.3 FF in eccentric orbit - unknown perigee/apogee time . . . 78

5 Spacecraft Formation Relative Attitude Determination . . . . . . . 81 


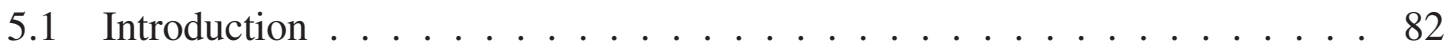

5.2 Relative Attitude Determination . . . . . . . . . . . . . . . . 84

5.2 .1 Covariance Analysis . . . . . . . . . . . . . . . . 87

5.2.2 Measurement Covariance - Parallel Case . . . . . . . . . . . 91

5.3 Relative Attitude Determination - Non-Parallel Case . . . . . . . . . . 93

5.3.1 Covariance Analysis - Non-Parallel Case . . . . . . . . . . . 96

5.3.2 Measurement covariance - Non-Parallel Case . . . . . . . . . . 97

5.4 Simulation . . . . . . . . . . . . . . . . . . . . 97

$5.4 .1 \quad$ Parallel Case . . . . . . . . . . . . . . . . . . 98

5.4 .2 Non-Parallel Case . . . . . . . . . . . . . . . . . . 102

6 A Weighted Measurement Fusion Kalman Filter Implementation for UAV Navigation ............................... 105

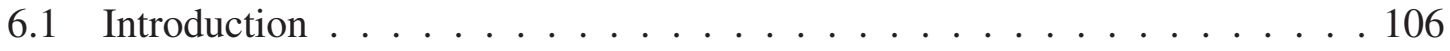

6.2 Problem Formulation . . . . . . . . . . . . . . . . . 108

6.3 Weighted Measurement Fusion . . . . . . . . . . . . . . . 111

6.4 Ellipsoid Error Probable . . . . . . . . . . . . . . . . . . . 114

6.5 Simulation . . . . . . . . . . . . . . . . . . . . 114

6.5.1 Scenario One: Accuracy Performance without GPS . . . . . . . 117

6.5.2 Scenario Two: Accuracy Performance with GPS available $\ldots . .118$

6.5.3 Ellipsoid Error Probable . . . . . . . . . . . . . . . . . 119

6.5 .4 Comparisons . . . . . . . . . . . . . . . . 120

6.5.5 Computational Loads . . . . . . . . . . . . . . . . . . . 120

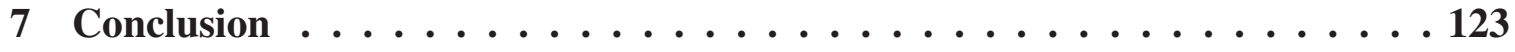




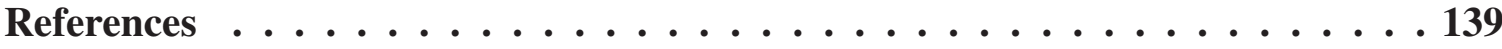

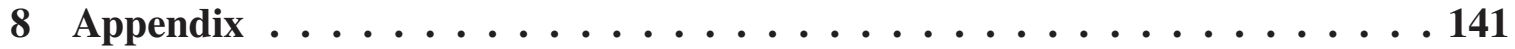

8.1 Linearization and covariance analysis in polar and cartesian coordinate . . . 141

8.1.1 Time derivative of state vectors in polar and cartersian coordinate . 141

8.1.2 Conversion of measurement noise covariance from polar to cartesian coordinate . . . . . . . . . . . . . . . . 142

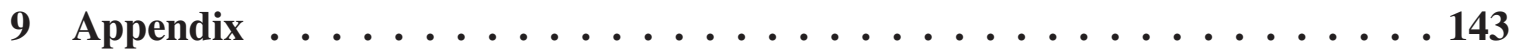

9.1 Linearization of orbital elements, and $f$ and $g$ function . . . . . . . . . 143

9.1.1 Partial Derivatives for semimajor axis, eccentricity, and eccentric anomaly . . . . . . . . . . . . . . . . . 143

9.1.2 Linearization of $\Delta t_{p}$ and $\Delta t_{a} \ldots \ldots \ldots \ldots \ldots$

9.1.3 Linearization of $f$ and $g$ functions. . . . . . . . . . . . 145 


\section{List of Figures}

2.1 (Left)Signal transmission between DBS and TRX. (Right) TRX's signal arrives at antenna

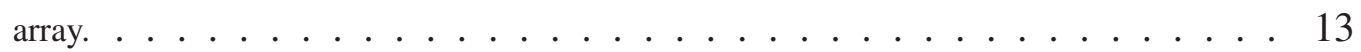

2.2 DBS ID signal and TRX response signal in an IRT period. . . . . . . . . . . . . . . 14

2.3 Relative position vector between two spacecraft. . . . . . . . . . . . 15

2.4 Illustration of signal transmission time delay . . . . . . . . . . . . . . 18

2.5 WLPS only - RMSE Performance. . . . . . . . . . . . . . . . 28

2.6 RMSE comparison of Azimuth only (1 DOA) versus Azimuth and Elevation $(2 \mathrm{DOA})$ measurements. . . . . . . . . . . . . . . . 30

(a) Low TOA-DOA measurement noise. . . . . . . . . . . . 30

(b) High TOA-DOA measurement noise. . . . . . . . . . . 30

2.7 Position RMSE comparison of GPS-only and GPS/WLPS, and three sigma boundaries plot. . . . . . . . . . . . . . . 33

(a) RMSE comparison with respect to number of spacecraft. . . . . . . 33

(b) GPS/WLPS position error with three sigma boundaries. . . . . . . 33

3.1 DG Est. Noise to Signal Ratio. . . . . . . . . . . . . . . . . . . . . 54

3.2 Measurement error wrt. distance. . . . . . . . . . . . . . . . . . 54

3.3 DG Est. RMSE. . . . . . . . . . . . . . . . . . 56

3.4 EKFRMSE . . . . . . . . . . . . . . . . . . . . . . . . 56 
3.5 Minimum and Maximum Mean RMSE Comparison between two filters with respect to measurement noise increment. . . . . . . . . . . . . 57

3.6 RMSE Comparison (Stable EKF). . . . . . . . . . . . . . . . . . . . 57

3.7 RMSE Comparison (Unstable EKf). . . . . . . . . . . . . . . . . . . 57

4.1 Estimation error and three sigma boundary plots for EKF and CKF . . . . . 66

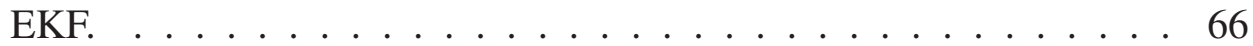

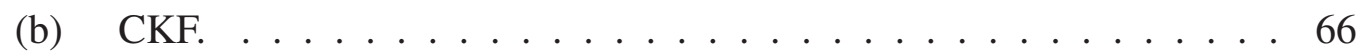

4.2 Change of covariance boundary in Kalman Filter process. . . . . . . . . . . . . 67

4.3 Applying perigee/apogee constraints after implementing EKF for high number of observations. . . . . . . . . . . . . . . . . . 6 67

4.4 CKF and EKF estimation performance comparison. . . . . . . . . . 75

(a) EKF's absolute position RMSE . . . . . . . . . . . . 75

(b) 3D plot for case C (unknown $\Delta t_{p}$ and $\left.\Delta t_{a}\right) \ldots \ldots \ldots$

(c) Performance ratio comparison at $\beta=0.4 \ldots 75$

(d) Performance ratio comparison at $\alpha=0.4 \ldots \ldots 75$

4.5 Estimation accuracy of CKF relative to that of EKF, for circular orbits . . . 77

(a) $\quad a=7000 \mathrm{~km} \ldots \ldots \ldots 77$

(b) $\quad a=11000 \mathrm{~km} \ldots \ldots \ldots \ldots \ldots$

4.6 Estimation accuracy of CKF relative to that of EKF, for eccentric orbits with known perigee/apogee times . . . . . . . . . . . . . 78

(a) $\quad a=7000 \mathrm{~km} \ldots \ldots \ldots \ldots \ldots$

(b) $\quad a=11000 \mathrm{~km} \ldots \ldots \ldots \ldots \ldots$

4.7 Estimation accuracy of CKF relative to that of EKF, for eccentric orbits with unknown perigee/apogee times . . . . . . . . . . . . 79

(a) $\quad a=7000 \mathrm{~km} \ldots \ldots \ldots \ldots \ldots$ 
4.8 Estimation accuracy of CKF relative to that of EKF, for eccentric orbits with unknown perigee/apogee times and initial guess errors, for $a=11000 \mathrm{~km} .79$

5.1 TRIAD method requires two pairs of LOS measurements. . . . . . . . . . . . 83

5.2 Measurements in three spacecraft formation. . . . . . . . . . . . . . . . 85

5.3 The line-of-sight measurement in three-spacecraft formation for non-parallel case. . . . . . . . . . . . . . . . . . . 94

5.4 Three Spacecraft Formation Configuration. . . . . . . . . . . . . . . . . . . . . . 98

5.5 Errors in the relative attitude $A_{d_{1}}^{d_{2}} \ldots \ldots \ldots \ldots$. . . . . . . . . . . 99

5.6 Errors in the relative attitude $A_{d_{3}}^{d_{1}} \ldots \ldots \ldots \ldots \ldots$

5.7 Errors in the relative attitude $A_{d_{3}}^{d_{2}} \ldots \ldots \ldots \ldots \ldots$

5.8 Th RMSE of $A_{d_{c}}^{d_{2}}$ with respect to change of $\theta_{1} \ldots \ldots \ldots \ldots$

5.9 Comparison between Analytical and Simulation Error with respect to measurement noise standard deviation. . . . . . . . . . . . . . . . . . . . . 101

5.10 Relative attitude error of $A_{1}^{2}$ in three sigma boundary for two Psi solution. . 103

(a) First Psi solution. . . . . . . . . . . . . . . . . 103

(b) Second Psi solution. . . . . . . . . . . . . . . . . . 103

6.1 Illustration of transmission between DBS and TRX. . . . . . . . . . . . . 109

6.2 WMFKF fuses the measurements and updates the states only at times $\left(t_{N}\right)_{i} \ldots \ldots \ldots$

6.3 Block diagram of the WMFKF algorithm. . . . . . . . . . . . . 111

6.4 Estimated position error (bold solid line) with three sigma boundary (dashdotted line) plot for $10 \mathrm{~km}$ range limits. . . . . . . . . . . . . . . 117

(a) Fusion Kalman Filter. . . . . . . . . . . . . . . . . 117

(b) Linear Kalman Filter. . . . . . . . . . . . . . . . . . 117 
6.5 Estimated position error (bold solid line) with three sigma boundary (dashdotted line) plot for $30 \mathrm{~km}$ range limits. . . . . . . . . . . . . 118

(a) Fusion Kalman Filter. . . . . . . . . . . . . . . . 118

(b) Linear Kalman Filter. . . . . . . . . . . . . . . . . . . . 118

6.6 Estimated position error (bold solid line) and three sigma boundary (dashdotted line) plot for 30km range limits with GPS available. . . . . . . . . 119

(a) Fusion Kalman Filter. . . . . . . . . . . . . . . . . . . . . 119

(b) Linear Kalman Filter. . . . . . . . . . . . . . . . . . . . . . 119

6.7 Error Probability Percentage for bot the MFKF and the WMFKF. . . . . 120 


\section{List of Tables}

2.1 Third and fourth spacecrafts' orbital elements. . . . . . . . . . . . 28

2.2 Four Spacecraft Configuration for different formation size. . . . . . . . . . 31

2.3 Performance comparison between different formation size. . . . . . . . . . 31

2.4 Performance comparison between high GPS noise and high WLPS noise. . 32

3.1 Spacecraft formation's configuration for short, medium and long range formation. ......................... 53

3.2 Comparing Mean RMSE in Absolute Position: DG Est. vs EKF. . . . . . . 53

3.3 Measurement noise's set configuration. . . . . . . . . . . . . 55

4.1 Spacecraft configuration. . . . . . . . . . . . 73

4.2 Asbsolute Position Estimation's Average RMSE of EKF. . . . . . . . . . . 76

6.1 Position of TRXs. . . . . . . . . . . . . . . 115

6.2 Comparison of number of multiplication required. . . . . . . . . . . . . 121 


\section{Preface}

The author has prepared the analytical and numerical solution, and simulation for the research work in each chapter. Also, the author has written four journal papers for publication and presented four conference for the results obtained in each chapter. Chapters two to five in this dissertation address the problem of spacecraft formation navigation. It is assumed that the relative positions are measured via a wireless local positioning system. Four different approaches were developed to estimate the spacecraft absolute and relative positions. These approaches are the extended Kalman filter, the constrained Kalman filter, the differential geometric filter, and the weighted measurement fusion Kalman filter. Chapters two to five where published in four different journals. The analytical work presented in chapter six presents an attitude estimation method for spacecraft formation. This work was published in the IEEE Aerospace conference. 


\section{Acknowledgements}

I would like to thank both my advisors, Dr. Ossama Abdelkhalik and Dr. Seyed A. (Reza)

Zekavat for their support. My research work towards my $\mathrm{PhD}$ degree would not have been successful without their invaluable experience and knowledge which they made available to me. Under their guidance, I have become more confident to advance my career in the future.

I would like to thank my committee, Dr. Chris E. Passerello who has assisted me in my research work, and also to Dr. Bo Chen for her support.

I would like also to thank all of my friends who have been my company during my stay in Houghton. My life would not be easy and joyful without their hospitality. It is a pleasure to have them around me. I am very pleased to have met with the Table Tennis members in St. Albert church who made my life more colorful every day.

Finally, I would like to express my greatest appreciation to my parents and family, who have supported my life and my decisions. Without their understanding and support, my career would not become successful. 


\section{Abstract}

Spacecraft formation flying navigation continues to receive a great deal of interest. The research presented in this dissertation focuses on developing methods for estimating spacecraft absolute and relative positions, assuming measurements of only relative positions using wireless sensors. The implementation of the extended Kalman filter to the spacecraft formation navigation problem results in high estimation errors and instabilities in state estimation at times. This is due tp the high nonlinearities in the system dynamic model. Several approaches are attempted in this dissertation aiming at increasing the estimation stability and improving the estimation accuracy.

A differential geometric filter is implemented for spacecraft positions estimation. The differential geometric filter avoids the linearization step (which is always carried out in the extended Kalman filter) through a mathematical transformation that converts the nonlinear system into a linear system. A linear estimator is designed in the linear domain, and then transformed back to the physical domain. This approach demonstrated better estimation stability for spacecraft formation positions estimation, as detailed in this dissertation.

The constrained Kalman filter is also implemented for spacecraft formation flying absolute positions estimation. The orbital motion of a spacecraft is characterized by two range extrema (perigee and apogee). At the extremum, the rate of change of a spacecraft's range vanishes. This motion constraint can be used to improve the position estimation accuracy. The application of the constrained Kalman filter at only two points in the orbit causes filter instability. Two variables are introduced into the constrained Kalman filter to maintain the stability and improve the estimation accuracy. An extended Kalman filter is implemented as a benchmark for comparison with the constrained Kalman filter. Simulation results show that the constrained Kalman filter provides better estimation accuracy as compared with the extended Kalman filter.

A Weighted Measurement Fusion Kalman Filter (WMFKF) is proposed in this dissertation. In wireless localizing sensors, a measurement error is proportional to the distance of the signal travels and sensor noise. In this proposed Weighted Measurement Fusion Kalman Filter, the signal traveling time delay is not modeled; however, each measurement is weighted based on the measured signal travel distance. The obtained estimation performance is compared to the standard Kalman filter in two scenarios. The first scenario assumes using a wireless local positioning system in a GPS denied 
environment. The second scenario assumes the availability of both the wireless local positioning system and GPS measurements. The simulation results show that the WMFKF has similar accuracy performance as the standard Kalman Filter (KF) in the GPS denied environment. However, the WMFKF maintains the position estimation error within its expected error boundary when the WLPS detection range limit is above $30 \mathrm{~km}$. In addition, the WMFKF has a better accuracy and stability performance when GPS is available. Also, the computational cost analysis shows that the WMFKF has less computational cost than the standard KF, and the WMFKF has higher ellipsoid error probable percentage than the standard Measurement Fusion method.

A method to determine the relative attitudes between three spacecraft is developed. The method requires four direction measurements between the three spacecraft. The simulation results and covariance analysis show that the method's error falls within a three sigma boundary without exhibiting any singularity issues. A study of the accuracy of the proposed method with respect to the shape of the spacecraft formation is also presented. 


\section{Chapter 1}

\section{Introduction}

\subsection{Overview}

The relative and absolute positions estimation of spacecraft formations is a fundamental task in many space missions. Relative position estimation plays an important role in Spacecraft Formation Flying (SFF) missions, a subject that has received a great deal of attention by researchers in recent decades. Some SFF missions require that multiple spacecraft in different orbits communicate without interruption, e.g., the satellites utilize the Computerized Ionospheric Tomography Radio Instrument in Space (CITRIS) under the Constellation Observing System for Meteorology, Ionosphere \& Climate (COSMIC) program, which is also known as the CITRIS-COSMIC system are required to communicate with each other to monitor the ionospheric irregularities [1]. Relative positions between satellites, such as Cluster and Cluster-II satellites that are launched by the European Space Agency, are estimated and controlled to support many collaborative tasks where satellites are required to maintain a specific formation in a continuous manner within the mission period [2].

The relative position estimation in spacecraft formation has been extensively studied by researchers. Ref. [3] and [4] have presented a spacecraft formation localization method based on the ranging measurement between spacecraft in a three-spacecraft formation. This ranging system is also known as the Autonomous Formation Flyer (AFF). The fundamental localization process of this system is similar to the Global Positioning System (GPS) localization, and it is able to provide high precision relative position 
estimation. However, complex hardware is required on each spacecraft. This system does not stand alone and requires a GPS system.

Given measurements of relative positions between two spacecraft, Ref. [5] proves the feasibility of estimating the orbits of the two spacecraft. A detailed observability analysis as well as a detailed implementation of a batch estimator were developed. Ref. [5] shows that the orbital elements estimation accuracy depends on the relative distance between spacecraft. Ref. [6] shows the feasibility of orbit navigation of two spacecraft using line-of-sight (LOS) measurements, and suggests that LOS measurements can be used for spacecraft formation navigation. Both Ref. [5] and [6] show that the inclination of the spacecraft orbits impacts the estimation accuracy. The system becomes unobservable in a few cases, such as the case when the two spacecraft are in the same zero inclination orbit plane $[5,6]$.

Ref. [7] presents the relative position and attitude estimation in a two-spacecraft formation using a Vision-Based Navigation System (VISNAV). A sensor installed on one spacecraft measures the line-of-sight (LOS) measurements of multiple beacons installed on the other spacecraft. The covariance study shows that the performance could be affected by the relative distance between spacecraft. The accuracy factors of the estimation depend on the number of beacons installed on the spacecraft. Three or more beacons are suggested to ensure the observability of the system [7].

Besides that, relative attitude estimation receives a great deal of interest from researchers. Missions that require relative attitude estimation include the spacecraft docking missions, chaser and target space missions [8], and clusters that perform their tasks in specific formations, such as Proba-3 [9] and LISA Pathfinder's missions [10, 11]. In these missions, the spacecraft are often required to maintain a specific orientation with respect to each other. The relative attitude determination between spacecraft is a fundamental task in these types of missions. Ref. [7] has presented both relative position and relative attitude estimation method based on VISNAV.

In addition, Ref. [12] introduces a Relative Attitude Determination (RAD) method for a three vehicle formation, using all three pairs of measurements between the three spacecraft. The relative attitude between two spacecraft is determined using an angle and a vector as the constraints. Then, the TRIAD method is used to find the relative attitude between the other two spacecraft. Althought the previous work showed that the covariance singularity due to the co-planar may be encountered [13], Ref. [12] showed that the 
proposed method does not process any singularity issue.

\subsection{Optimal State Estimation and Navigation}

The Kalman Filter (KF) [14] is a sequential linear estimation algorithm capable of estimating both observable and unobservable variables in real time. It has been extensively studied by researchers for application in several areas, such as navigation, economic, and earth sciences [15]. The fundamental theory of KF consists of two processes: (1) Predicting, and (2) Updating. The KF predicts the possible observation data based on the estimated parameters. Then, the estimated parameters are updated by integrating the predicted observation with the measured observation. The derivation of KF has been extended to incorporate with different case studies, such as nonlinear system, bad initial condition estimation, non-Gaussian error, and large data estimation.

The Extended Kalman Filter (EKF), a sequential nonlinear estimation algorithm, has been widely implemented to estimate absolute and relative positions, and the relative attitude of spacecraft in formation flying $[7,16]$. However, the accuracy of the estimated initial condition affect the EKF stability. Furthermore, the linearization process on the nonlinear model results the EKF becoming unable to guarantee a stable estimation all the time.

The Unscented Kalman Filter (UKF) has been implemented for relative attitude and position estimation in SFF $[17,18]$. The UKF has better robustness to initial condition error compared to the EKF [19]. Besides that, it does not assume the measurement errors are Gaussian noise. However, its computational time is higher than a standard EKF [20]. If compared to the standard EKF, the UKF has a faster convergence rate, but the process time required for each update is longer than the EKF [21]. The Batch filter [22], an offline filtering method, has been implemented in Ref. [5] for absolute position estimation using the relative position measurement. Both studies in references [16] and [5] show that the configuration of SFF affects the stability and the accuracy of estimation.

Researchers are always interested in improving the performance of the Kalman Filter, especially the estimation accuracy. The Constrained Kalman Filter (CKF) was introduced for estimation when state variables are required to satisfy constraints [23, 24]. Both equality and inequality constraints can be handled by the CKF. Several CKF algorithms have been proposed in the literature, e.g., the perfect measurement approach $[25,26]$, and 
the projection approach [27]. Some of the CKF methods may require special derivations for different problems, such as the reduction method [28] and the Norm-Constrained method in Ref. [29].

The constraint is handled as a perfectly known measurement in the perfect measurement approach. The constraint is grouped with other sensor measurements to construct a modified measurement vector, which affects the calculation of the Kalman gain [23]. Adding a perfect measurement (constraint) results in a singular noise covariance matrix. This singularity in the noise covariance may lead to a divergence in the estimation error. This fact was pointed out in references [30] and [31], despite that, the original work of Kalman [14] presents an example in which a perfect measurement was used [23]. To overcome this singularity problem, Ref. [32] presents an extended maximum likelihood method for computing the constraint gain.

In the projection approach, the constraint estimation is implemented by projecting the unconstrained state estimate onto the constraint surface. In general, the constraint update is applied after the standard Kalman filter update [23]. Ref. [23] has presented three different constraint update methods that can be used for the projection approach. However, different approximated solutions may be obtained from each method because the constraint gain is not correlated with the sensor measurement [23]. The projection approach has also been extended for inequality constraint [33] and nonlinear constraint [27] problems. The Norm-Constrained method presented in Ref. [29] guarantees non-singular estimation and correlates both the measurement and the constraint. However, the derivation of the Kalman gain is specific to the attitude estimation problem, and becomes rather complex when applied for the problem presented in this chapter. The reduction method requires that one or more state(s) be expressed as a linear function(s) of the other states. However, this method may not be applicable for the cases where the constraint states are independent from each other. Ref. [34] presents a Smoothly CKF (SCKF) where the constraint update via carried out iteratively until an end criteria is met. Ref. [34] shows that the SCKF outperforms the Iterated EKF in terms of consistency. Besides that, researchers are interested in reducing the computational load for the estimation process in multisensor systems. One way to process measurements in a multisensor system is to combine all the measurements into a single observation vector in the KF. However, the computational load increases as the number of measurements increases [35]. Ref. [36] presents a State-Vector Fusion (SVF) method in which each 
measurement is processed by its own local filter simultaneously. Then, the updated estimated states and the predicted covariances are fused together [37]. The Measurement Fusion (MF) method, introduced in Ref. [38], fuses multiple measurements and then the $\mathrm{KF}$ is applied to the result. Ref. [39] has analytically shown that, under certain conditions, the MF method is similar to the standard KF. Both SVF and MF methods require less computational load compared to the standard KF [38]. However, the derivation of these fusion methods assumes uncorrelated measurement noise for multisensor systems $[37,40]$. In most of the multisensor systems, the sensors' noises are correlated due to the interference signal between sensors [40]. Ref. [41] has presented a weighted MF method in which the input and measurement noises could be correlated. In addition, the computational load of the weighted MF method is significantly lower than the KF [41].

The Differential Geometric (DG) Theory has been widely implemented in the control and guidance research area $[42,43]$. In calculus, the Differential Geometry represents the linear approximation of a smooth curve [44]. Ref. [45] and [46] introduced the Differential Geometric approach into the missile tracking applications. In Ref. [45], the system model is transformed from the arc length domain into the time domain; then the guidance law is applied. Both papers have shown the feasibility of implementing the DG for missile guidance. The Differential Geometric approach for nonlinear systems has been extended recently for the nonlinear estimation purpose [47]. The DG estimation avoids the linearization step in the EKF. In DG estimation, the nonlinear dynamics are mapped to a linear domain, where a linear estimator can be implemented. Then, the inverse transformation is applied to the estimator [47]. Ref. [47] details how the DG estimation can be implemented when the number of available measurements are too few to handle all the system nonlinearities, and shows that both the optimal control and the linear filtering approaches can be applied in the mapped linear domain.

\subsection{Navigation/Localization Sensors}

Radio interferometer has been used as a spacecraft position tracking system in many applications. Ref. [48] presents different angular measurement technique for spacecraft navigation based on the radio interferometer. Besides that, the Laser Interferometer, which provides a long range line-of-sight measurement has been under development as an instrument for spacecraft formation guidance, navigation, and control [11]. 
The AFF has been introduced as a relative position measurement instrument for spacecraft formation flying in recent decades $[3,4]$. The AFF positioning system is similar to the GPS system. However, it also requires additional instrumentation to provide the absolute positioning. Besides that, the Vision-Based Navigation System (VISNAV) has been developed by a group in Texas A\&M University [49]. This system is capable of providing the line-of-sight (LOS) measurement of the beacon attached on a target. The VISNAV consists of a Position Sensing Diode (PSD) sensor and an array of LED source beacons installed on a plate. The PSD detects the energy of the light source emitted by the beacons. Then the PSD generates a current flow to four terminals that are installed on the PSD plate. At that point, the centroid of the current flow is determined to measure the direction of the energy source. The application of this sensor has been studied in several areas, such as spacecraft docking, air refueling and spacecraft formation flying problems [7, 50, 51].

The one-way ranging system requires clock synchronization between the two nodes [52], e.g., the Global Positioning System (GPS) [53]. For example, Ultra WideBand (UWB) communication for spacecraft formation navigation presented by Ref. [52] requires clock synchronization between transmitter and receiver, to allow the receiver to compute the relative distance by receiving the pulse signal sent by the receiver.

On the other hand, two way transmission does not require clock synchronization. The Two-Way Satellite Time and Frequency Transfer (TWSTFT) method has been implemented for decades. The TWSTFT allows two ground stations to measure the round trip signals that are transmitted from the ground to the satellite, and then back to a ground station [54-56]. The TWSTFT method has shown that if both the transmitted and received signals are reciprocal, then the error due to the signal transmission time delay is canceled out. Despite that fact, there are few other non-reciprocal errors which cannot be avoided. However, in general, it has been shown that the ranging accuracy obtained from this method can be up to 1.5 nanoseconds [57].

In this dissertation, spacecraft formation navigation is studied based on the Wireless Local Positioning System (WLPS) [58]. The WLPS is a localization system capable of providing relative localization between two nodes [58]. The WLPS consists of two components: the dynamic base station (DBS) and transponder (TRX). The DBS installed on one spacecraft measures the relative position of the TRX installed on another spacecraft, which is located in its coverage area via Time-of-Arrival (TOA) and Direction-of-Arrival (DOA) estimation. The TOA is the time needed by the transmitted signal to travel from DBS to 
TRX and back from TRX to DBS. The signal arrives at the two-dimensional antenna array in DBS, enabling the system to compute DOA (azimuth and elevation angles).

\subsection{Objectives of this Dissertation}

The main research objective is to implement the Wireless Local Positioning System (WLPS) into the SFF and apply different estimation methods to enhance the estimation performance. The WLPS consists of two components: the dynamic base station (DBS) and transponder (TRX). The DBS installed on one spacecraft measures the relative position of the TRX installed on another spacecraft, which is located in its coverage area via Time-of-Arrival (TOA) and Direction-of-Arrival (DOA) estimation. The TOA is the time needed by the transmitted signal to travel from DBS to TRX and back from TRX to DBS. The signal arrives at the two-dimensional antenna array in DBS, enabling the system to compute DOA (azimuth and elevation angles).

The research objectives are shown as follows:

1. Implement the WLPS into SFF navigation to improve the estimation accuracy [59];

2. Implement the Differential Geometric Filter into the SFF navigation using the WLPS as measurement [60];

3. Apply the constraint at orbit's perigee and apogee point to improve the overall estimation accuracy [61];

4. Propose an alternate attitude determination method for a three-spacecraft formation flying [62]; and,

5. Implement a measurement fusion method into the Kalman Filter to improve the estimation performance [63].

\subsection{Organization of this Dissertation}

The dissertation is organized as follows: Chapter Two presents the Spacecraft Formation Orbit Estimation using WLPS-based localization. Chapter Three presents the 
Implementation of a Differential Geometric Filter. for Spacecraft Formation Orbit. Chapter Four presents the Constraint Estimation of Spacecraft Positions. Chapter Five presents the Spacecraft Formation Relative Attitude Determination. Chapter Six presents a Weighted Measurement Fusion Kalman Filter Implementation. Finally, Chapter Seven presents the conclusion of the dissertation objectives. 


\section{Chapter 2}

\section{Spacecraft Formation Orbit Estimation using WLPS-based Localization}




\subsection{Introduction}

The relative and absolute positions estimation of spacecraft formations is a fundamental task in many space missions. ${ }^{*}$ Relative position estimation plays an important role in Spacecraft Formation Flying (SFF) missions, a subject that has received a great deal of attention by researchers in recent decades. Some SFF missions require that multiple spacecraft in different orbits communicate without interruption, e.g., satellites in the CITRIS-COSMIC system are required to communicate with each other to monitor the ionospheric irregularities [1]. Relative positions between satellites, such as Cluster and Cluster-II satellites launched by the European Space Agency, are estimated and controlled to support many collaborative tasks where satellites are required to maintain a specific formation in a continuous manner within the mission period [2].

Several relative positions estimation systems have been developed. The Autonomous Formation Flyer (AFF) technology was developed for SFF [3, 4], where each spacecraft is equipped with a communication system to localize other spacecraft in the formation. This system provides high precision estimates for relative positions. A relative position and attitude estimation through a Vision Based Navigation system (VISNAV) has been studied extensively in the literature [7, 49-51]. The VISNAV enables one spacecraft to measure the line-of-sight (LOS) measurements of the other spacecraft. Estimation performance of this technique is a function of the relative distance of spacecraft and the number of beacons installed on the spacecraft [7].

Given measurements of relative positions between two spacecraft, Ref. [5] proves the feasibility of estimating the orbits of the two spacecraft. A detailed observability analysis as well as a detailed implementation of a batch estimator were developed. Ref. [5] shows that the orbital elements estimation accuracy depends on the relative distance between spacecraft. Ref. [6] shows the feasibility of orbit navigation of two spacecraft using line-of-sight (LOS) measurements, and suggests that LOS measurements can be used for spacecraft formation navigation. Both Ref. [5] and [6] show that the inclination of the spacecraft orbits impacts the estimation accuracy. The system becomes unobservable in a few cases, such as the case when the two spacecraft are in the same zero inclination orbit plane $[5,6]$.

*The material contained in this chapter was previously published in the International Journal of Navigation and Observation. 
Ref. [52] presents a study on the implementation of Ultra WideBand (UWB) communication for spacecraft formation navigation. A transmitter, at one spacecraft, sends a pulse to a receiver installed on another spacecraft. Next, the receiver computes the relative distance between the two spacecraft through measuring the difference between the signal transmission time and arrival time. In this technique, a synchronization between the transmitter and receiver clocks is required to allow precise range measurement.

The one-way ranging system requires clock synchronization between the two nodes [52], e.g. the Global Positioning System (GPS) [53]. Sources of measurement error include the signal transmission time delay which reduces the accuracy performance of the range measurement. The Two-Way Satellite Time and Frequency Transfer (TWSTFT) method has been implemented for decades. The TWSTFT allows two ground stations to measure the round trip signals that are transmitted from the ground to the satellite, and then back to a ground station [54-56]. The TWSTFT method has shown that if both the transmitted and received signals are reciprocal, then the error due to the signal transmission time delay is canceled out. Despite that fact, there are few other non-reciprocal errors which cannot be avoided. However, in general, it has been shown that the ranging accuracy obtained from this method can be up to 1.5 nanoseconds [57].

When an observing spacecraft transmits a signal to a target spacecraft, the signal transmission time delay causes errors in the measured relative position [64, 65]. Ref. [66] shows that the signal transmission time delay can be computed if either the observer or the target is stationary. Also, the TWSTFT has shown that the signal transmission time delay between ground stations and spacecraft can be omitted [54-56]. However, when both observer and target spacecraft are moving, the complexity of estimating the signal transmission time delay increases. Different modifications of filtering methods have been proposed in the literature to compensate the measurement errors due to the time delay $[67,68]$. In Ref. [69], a closed form time delay approximation has been proposed using a Taylor series expansion. Ref. [69] shows that, in some cases, the state estimate error does not converge within the covariance boundary if the time delay is not modeled.

In this chapter, the orbits of two or more spacecraft in a formation are estimated through the implementation of a Wireless Local Positioning System (WLPS) that enables relative localization [58]. The WLPS consists of two components: the dynamic base station (DBS) and transponder (TRX). The DBS installed on one spacecraft measures the relative position of the TRX installed on another spacecraft, which is located in its coverage area 
via Time-of-Arrival (TOA) and Direction-of-Arrival (DOA) estimation. The TOA is the time needed by the transmitted signal to travel from DBS to TRX and back from TRX to DBS. The signal arrives at the two-dimensional antenna array in DBS, enabling the system to compute DOA (azimuth and elevation angles).

This chapter investigates:

(1) The feasibility of absolute position estimation of spacecraft formation flying using only one-dimensional DOA (1-DOA) in the WLPS. The 1-DOA WLPS uses one dimension antenna arrays to measure the range and only the azimuth angle. The two-dimensional DOA (2-DOA) WLPS uses two dimension antenna arrays to measure range and both azimuth and elevation angles. It is technologically more complicated compared to the 1-DOA WLPS;

(2) The absolute position estimation of spacecraft in formation using 2D WLPS, taking into consideration the signal time delay. One implementation issue with the WLPS is the time delay that is due to the processing time in the WLPS and due to the signal transmission between different spacecraft nodes. Even though the time delay due to signal transmission is very small, the high velocity of spacecraft makes it non-negligible in some formation scenarios; and

(3) The estimation performance of a GPS standalone system is compared to a combined GPS and WLPS.

Simulations are conducted to investigate how the position estimation performance is affected by the number of spacecraft in the formation, the size of the formation, the WLPS and GPS measurement noise variance, and the altitudes of spacecraft. The accuracy and the speed of convergence of the estimator is numerically studied. The Extended Kalman Filter (EKF) is implemented in all studies presented in this chapter.

The proposed study is critical for the implementation of localization sensors for many applications, including for space-based solar power transfer applications [70, 71]. In the futuristic space-based solar power harvesting techniques proposed in Ref. [70] and [71], the position of multiple satellites in charge of collection of solar power, should be properly estimated to maintain synchronized solar power transfer to an energy collecting unit on the earth. This study also has applications for deep space multi-spacecraft missions when GPS is not available.

This chapter is organized as follows: Section 2.2 presents an overview on WLPS. Section 2.3 presents the dynamics, the time delay modeling, and GPS mathematical model. 
Section 2.2 presents the EKF implementation. Section 2.5 discusses the simulation results.

\subsection{Wireless Local Positioning System (WLPS)}

The WLPS consists of two basic components [58]: A dynamic base station (DBS) and a transponder (TRX). Each DBS is capable of localizing TRXs that are located in its coverage area via TOA and DOA measurements, as shown in Figure 2.1. The DBS periodically broadcasts an identification (IDR) signal once every ID Request Repetition Time (IRT) as shown in Figure 2.2. A TRX that falls within the DBS coverage area receives the IDR signal and transmits a response signal that includes its own ID back to the DBS within the IRT period. The ID of each TRX allows the DBS to distinguish one TRX from another. It also allows the DBS to easily track multiple TRXs located in its coverage area.

As shown in Figure 2.2, the range of TRX is measured by comparing the TOA of the signal from the TRX at the DBS receiver and the time of transmission of the signal from the DBS transmitter. The processing time estimate can be included in the signal packet transmitted from TRX to DBS in order to allow DBS to correctly measure the range. The DBS, equipped with antenna arrays, allows DOA estimation and beamforming. In addition, beamforming enhances the performance of the DBS by reducing the interference effects [72]. The DOA is measured by each spacecraft relative to its body fixed coordinate system. In this chapter, we assume that the attitudes of all spacecraft are known. Hence, the DOA measurements can be computed relative to a fixed reference frame.
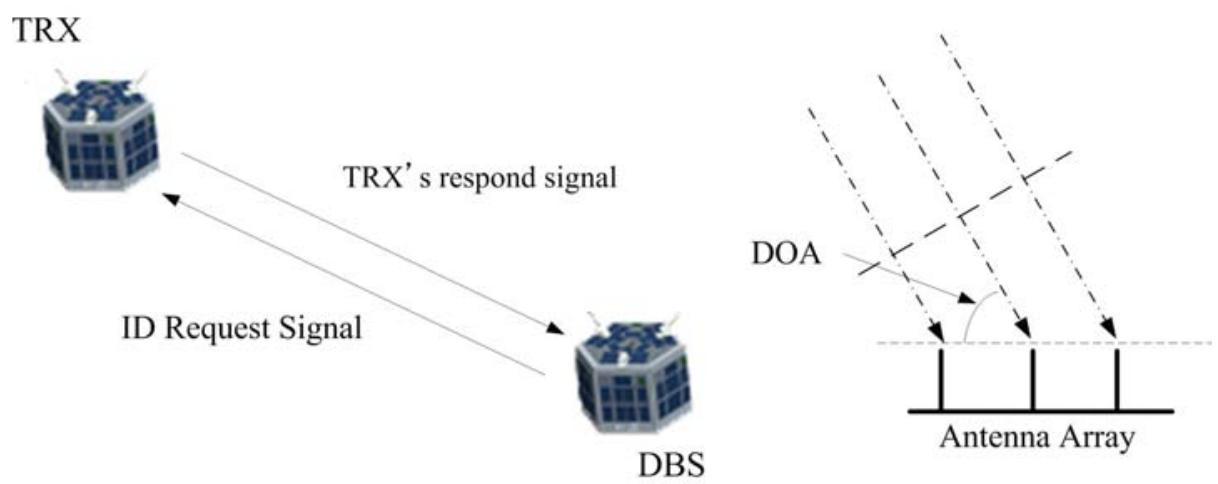

Figure 2.1: (Left)Signal transmission between DBS and TRX. (Right) TRX's signal arrives at antenna array. 


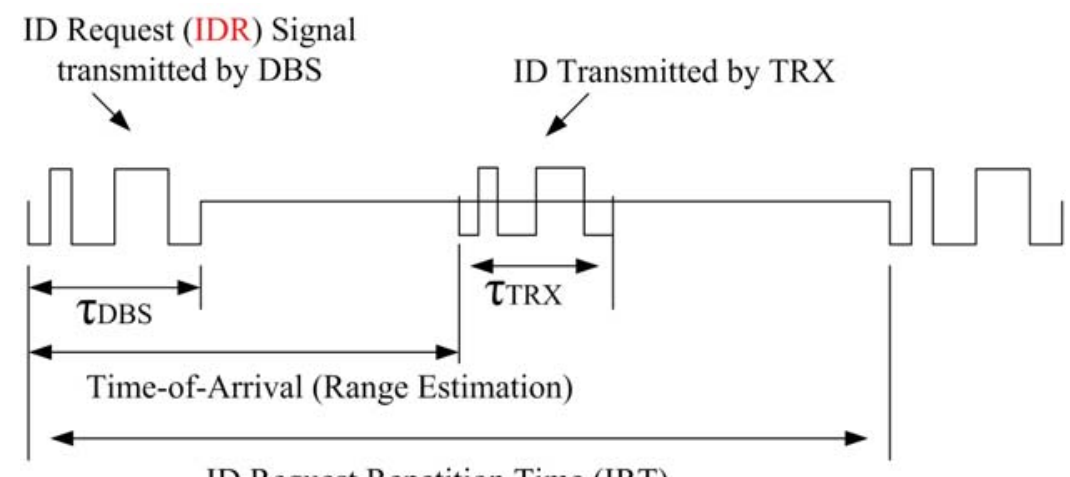

ID Request Repetition Time (IRT)

Figure 2.2: DBS ID signal and TRX response signal in an IRT period.

Thus, a WLPS allows single node positioning. In other words, each node equipped with a DBS can independently localize the TRXs located in its coverage area and its field-of-view (FOV). Now, if all spacecraft are equipped with both DBS and TRX, each spacecraft can find the position of other spacecraft located in its FOV and coverage area. The position information across multiple spacecraft can be fused to improve the localization performance [73].

\subsection{System Model}

In this section, the state and measurement models are derived. The estimated states are the spacecrafts' absolute positions and their velocity vectors. In this chapter, we assume that the spacecrafts' orientations are known, and hence the WLPS measurements can be expressed in the inertial reference frame. The spacecraft orientation is represented by the Direction Cosine Matrix [74].

\subsubsection{State Model}

The estimated state vector, $\hat{\mathbf{x}}$, and its time derivative vector, $\dot{\hat{\mathbf{x}}}$, for a formation of $n$ spacecraft, are defined as:

$$
\begin{aligned}
\hat{\mathbf{x}} & =\left[\begin{array}{llllllll}
\mathbf{r}_{1}^{T} & \mathbf{r}_{2}^{T} & \ldots & \mathbf{r}_{n}^{T} & \dot{\mathbf{r}}_{1}^{T} & \dot{\mathbf{r}}_{2}^{T} & \ldots & \dot{\mathbf{r}}_{n}^{T}
\end{array}\right]^{T}, \\
\dot{\hat{\mathbf{x}}} & =\left[\begin{array}{llllllll}
\dot{\mathbf{r}}_{1}^{T} & \dot{\mathbf{r}}_{2}^{T} & \ldots & \dot{\mathbf{r}}_{n}^{T} & \dot{\mathbf{r}}_{1}^{T} & \dot{\mathbf{r}}_{2}^{T} & \ldots & \dot{\mathbf{r}}_{n}^{T}
\end{array}\right]^{T},
\end{aligned}
$$




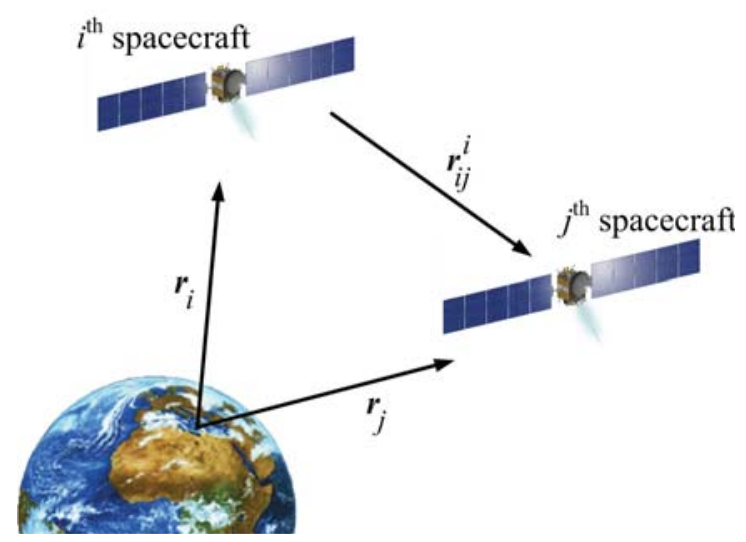

Figure 2.3: Relative position vector between two spacecraft.

The $i^{\text {th }}$ spacecraft's absolute position is $\mathbf{r}_{i}=\left[\begin{array}{lll}r_{i, x} & r_{i, y} & r_{i, z}\end{array}\right]^{T}$, the velocity vector is $\dot{\mathbf{r}}_{i}=\left[\begin{array}{lll}\dot{r}_{i, x} & \dot{r}_{i, y} & \dot{r}_{i, z}\end{array}\right]^{T}$, and the acceleration vector is $\ddot{\mathbf{r}}_{i}=\left[\begin{array}{lll}\ddot{r}_{i, x} & \ddot{r}_{i, y} & \ddot{r}_{i, z}\end{array}\right]^{T}$ (all in the Earth Centered Inertial (ECI) frame). The sign $\hat{.}$ refers to the estimated values.

The spacecraft's motion with respect to the earth's center is represented by the two-body model $[75,76]$ :

$$
\ddot{\mathbf{r}}=\frac{-\mu}{r^{3}} \mathbf{r}
$$

where $\mu$ is the Earth gravitational constant, the vectors, $\mathbf{r}$ and $\ddot{\mathbf{r}}$ are the absolute position and acceleration vectors of the spacecraft, respectively, and $r$ denotes the magnitude of the vector $\mathbf{r}$.

\subsubsection{Measurements Model}

In this study, we assume each spacecraft is equipped with both DBS and TRX. Thus, it can localize other spacecraft and can be localized by other spacecraft.

In Figure 2.3, $\mathbf{r}_{i}$ and $\mathbf{r}_{j}$ represent the $i^{t h}$ and the $j^{\text {th }}$ spacecraft absolute position vectors expressed in the ECI frame. The relative position vector of the $j^{t h}$ spacecraft observed by 
the $i^{\text {th }}$ spacecraft that is equipped with DBS is:

$$
\begin{gathered}
\mathbf{r}_{i j}=\mathbf{r}_{j}-\mathbf{r}_{i} \\
\mathbf{r}_{i}=\left[\begin{array}{lll}
r_{i, x} & r_{i, y} & r_{i, z}
\end{array}\right]^{T} \\
\mathbf{r}_{j}=\left[\begin{array}{lll}
r_{j, x} & r_{j, y} & r_{j, z}
\end{array}\right]^{T}
\end{gathered}
$$

If the orientation of the spacecraft is known, the relative position vector, $\mathbf{R}_{i j}$, expressed in the $i^{\text {th }}$ spacecraft reference frame would be:

$$
\mathbf{R}_{i j}=C_{i} \mathbf{r}_{i j}
$$

where $C_{i}$ is the Direction Cosine Matrix (also known as Attitude Matrix [22]) of the $i^{t h}$ spacecraft relative to the ECI frame. Let $h_{i j}^{T O A}$ be the time of the ID signal transmission, as the ID signal travels from the DBS node $i$, received by the TRX node $j$, transmitted again from the TRX node $j$, until received back at the DBS node $i$. Assume, for now, that the both DBS $i$ and TRX $j$ are stationary, then we can write:

$$
\begin{aligned}
h_{i j}^{T O A} & =2 \times\left\|\mathbf{r}_{i j}\right\| / c \\
& =2 \times\left\|\mathbf{r}_{j}-\mathbf{r}_{i}\right\| / c
\end{aligned}
$$

where $\mathbf{r}_{j}$ is the position of node $j$ at the time it receives the ID signal, $c$ is the speed of light and $\|$.$\| refers to the magnitude of vector. In the above equation, we assumed zero$ processing time at the TRX. Let $\mathbf{R}_{i j}=\left[\begin{array}{lll}R_{i j, x} & R_{i j, y} & R_{i j, z}\end{array}\right]^{T}$, then the DOA between the two nodes $i$ and $j$ is given by the two measurements:

$$
\mathbf{h}_{i j}^{D O A}=\left[\begin{array}{c}
\theta_{i j} \\
\phi_{i j}
\end{array}\right]=\left[\begin{array}{c}
\tan ^{-1}\left(\frac{R_{i j, y}}{R_{i j, x}}\right) \\
\tan ^{-1}\left(\frac{R_{i j, z}}{\sqrt{R_{i j, x}^{2}+R_{i j, y}^{2}}}\right)
\end{array}\right]
$$

Here, $\theta_{i j}$ is the relative azimuth angle and $\phi_{i j}$ is the relative elevation angle between the two nodes $i$ and $j$. Using Eqs. (2.8) and (2.9), we can express the WLPS measurement 
between the $i^{\text {th }}$ and the $j^{\text {th }}$ nodes as:

$$
\tilde{\mathbf{y}}_{i j}=\left[\begin{array}{l}
h_{i j}^{T O A} \\
\mathbf{h}_{i j}^{D O A}
\end{array}\right]+v_{W L P S, i j}
$$

where $v_{W L P S, i j}$ denotes WLPS measurement noise, which is assumed to be zero-mean Gaussian with the covariance matrix $E\left[v_{W L P S, i j} v_{W L P S, i j}^{T}\right]=\Re_{W L P S, i j}$.

The observation matrix of the nonlinear measurement model in Eq. (2.10), is linearized using the first order Taylor series expansion, which corresponds to [14, 22]:

$$
\mathbf{h}(\mathbf{x}) \simeq \mathbf{h}(\hat{\mathbf{x}})+\left.\frac{\partial \mathbf{h}}{\partial \mathbf{x}}\right|_{\mathbf{x}=\hat{\mathbf{x}}}(\mathbf{x}-\hat{\mathbf{x}})
$$

where $\mathbf{h}(\mathbf{x})$ and $\mathbf{h}(\hat{\mathbf{x}})$ are the nonlinear measurement models and they are expressed in terms of the true state vector, $\mathbf{x}$, and the estimated state vector, $\hat{\mathbf{x}}$ respectively. In addition, $\frac{\partial \mathbf{h}}{\partial \mathbf{x}} \equiv H$, is the jacobian matrix of the nonlinear measurement model, (Eq. (2.10)), which is also known as the sensitivity matrix. Here, the sensitivity matrix for Eq. (2.10), $H_{W L P S}$ is given as:

$$
H_{W L P S}=\left[\begin{array}{lll}
-\frac{\partial \mathbf{h}}{\partial \mathbf{r}} & \frac{\partial \mathbf{h}}{\partial \mathbf{r}} & \mathbf{0}_{3 \times 6}
\end{array}\right]
$$

where,

$$
\frac{\partial \mathbf{h}}{\partial \mathbf{r}} \equiv\left[\begin{array}{ccc}
r_{i j, x} / r_{i j} & r_{i j, y} / r_{i j} & r_{i j, z} / r_{i j} \\
\frac{-\sin (\theta)}{r_{i j} \cos (\phi)} & \frac{-\cos (\theta)}{r_{i j} \cos (\phi)} & 0 \\
\frac{-\cos (\theta) \sin (\phi)}{r_{i j}} & \frac{-\sin (\theta) \sin (\phi)}{r_{i j}} & \frac{-\cos (\phi)}{r_{i j}}
\end{array}\right] C_{i}
$$

Here, $H_{W L P S}$ is derived assuming that we have only two spacecraft in the formation. However, the procedure can be extended to any number of spacecraft.

\subsubsection{Time Delay Modeling}

Time delay estimation has been extensively studied in the literature. The wide separation between spacecraft and the high velocity of spacecraft may result in a significant error in position measurements due to the signal transmission time delay. One approach to address time delay error is to consider the time delay as a Gaussian random variable, and its effect can be removed through Kalman Filter implementation. However, Ref. [77] shows that the error distribution due to the transmission time delay is not always a normal distribution. 


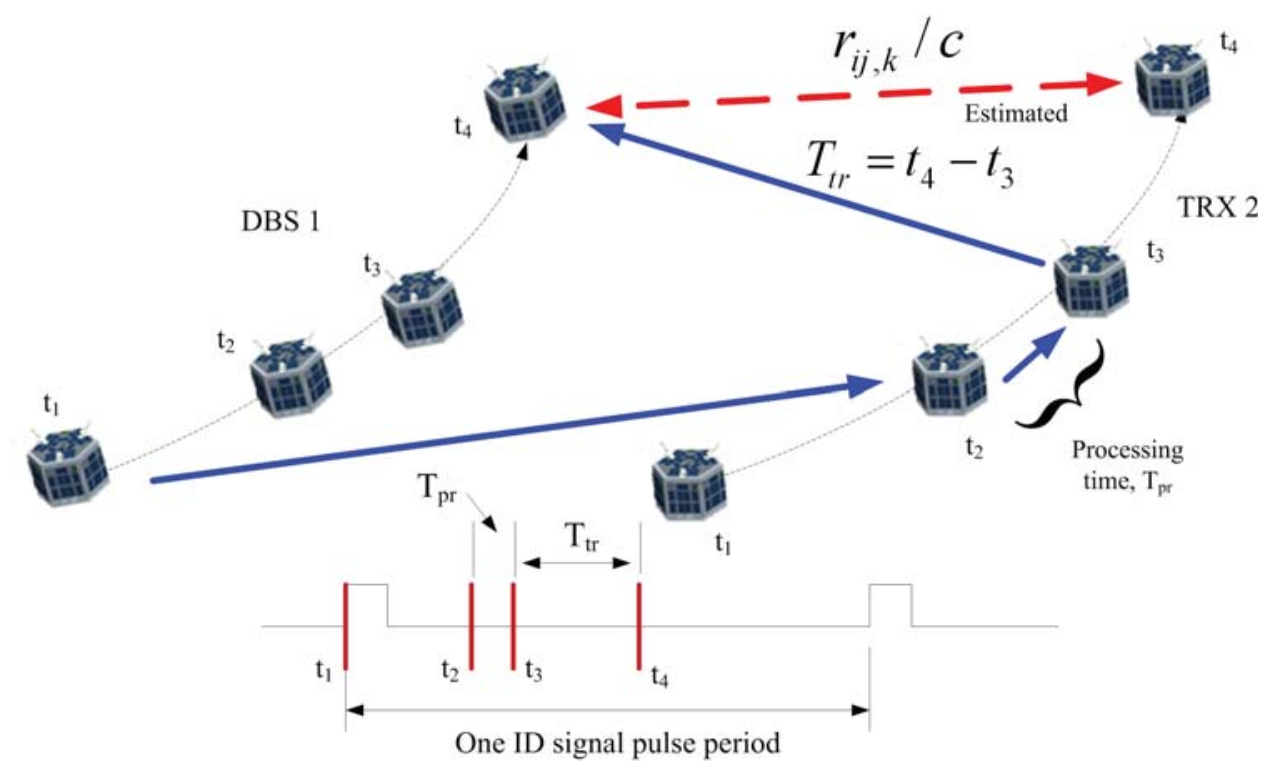

Figure 2.4: Illustration of signal transmission time delay

Ref. [69] presents an approximation method to model the signal transmission time delay between two spacecraft and its effect on elative position estimation, for one-way signal trips. States of past time are expanded as functions of the states at current time using the Taylor series expansion [69]. The WLPS measures the TOA of the round trip signal between the DBS and the TRX. Figure 2.4 illustrates the time delay due to the WLPS processing time and due to signal transmission. The true signal transmission path is described in the solid line. The dashed line is the signal path if the time delay is neglected (both DBS and TRX are stationary, Eq. (2.8), or moving at with low velocities).

In Figure 2.4, the DBS transmits its ID signal to TRX at time $t_{1}$. The TRX receives the signal at time $t_{2}$ and it requires $T_{p r}$ time to process the signal. Then, the TRX transmits the signal back to the DBS at time $t_{3}$ and the DBS recieves the signal at time $t_{4}$. When the DBS receives the ID signal at time $t_{4}$, it measures the Time-of-Arrival (TOA) of the signal $\left(t_{4}-t_{1}\right)$. The TOA is measured at the DBS receiver, thus, the clock synchronization between DBS and TRX is not required. It is to be noted that $T_{p r}$ can be determined, offline or online, by computing the clock pulse needed to process each DBS signal. In this chapter, we assume $T_{p r}$ is a known constant.

However, it is important to note that both spacecraft have travelled from their original position at time $t_{1}$ to a new position at time $t_{2}$ when the signal is transmitted from DBS to TRX. This is also applied for the signal transmission between time $t_{3}$ and $t_{4}$. Let the $i^{t h}$ 
spacecraft equipped with DBS and $j^{t h}$ spacecraft equiped with TRX; the round trip TOA measurement shown in Figure 2.4 can be expressed as:

$$
\tilde{\mathbf{y}}_{\text {delay }, T O A}=\left\|\mathbf{r}_{i, t_{4}}-\mathbf{r}_{j, t_{3}}\right\| / c+\left\|\mathbf{r}_{j, t_{2}}-\mathbf{r}_{i, t_{1}}\right\| / c+T_{p r}+v_{d, T O A}
$$

where $v_{d, T O A}$ is the measurement noise.

All positions of both spacecraft at $t_{1}, t_{2}$, and $t_{3}$ need to be expressed in terms of the positions at time $t_{4}$. By adapting the strategy developed in Ref. [69], the estimated round trip TOA based on Eq. (2.14) is given as:

$$
\hat{\mathbf{y}}_{\text {delay }, T O A}=\left\|\mathbf{r}_{i, k}-\mathbf{r}_{j, k-1}\right\| / c+\left\|\mathbf{r}_{j, k-2}-\mathbf{r}_{i, k-3}\right\| / c+T_{p r}
$$

where $t_{3} \equiv k-1, t_{2} \equiv k-2, t_{1} \equiv k-3$.

In Figure 2.4, the Time-of-Flight (TOF) of signal transmission between $i^{t h}$ and $j^{t h}$ spacecraft defined in Ref. [69], is presented as $T_{t r}$. Let $r_{i j, k}$ be the distance between DBS and TRX at time $t_{4}$, and $T_{t r}=r_{i j, k} / c+\tau$. Then, we can write:

$$
\begin{aligned}
r_{i j, k}+\tau c & =T_{t r} \times c \\
& =\sqrt{\left(\mathbf{r}_{j, k-1}-\mathbf{r}_{i, k}\right)^{T}\left(\mathbf{r}_{j, k-1}-\mathbf{r}_{i, k}\right)}
\end{aligned}
$$

It is noted that the true value of $T_{t r}$ is unknown, and we can only compute an estimate for the transmission time. The position of $j^{t h}$ spacecraft at time $t_{3}$ can be estimated via the first order Taylor series expansion, which is given as:

$$
\mathbf{r}_{j, k-1} \simeq \mathbf{r}_{j, k}-T_{t r} \mathbf{v}_{j, k}
$$

where $\mathbf{v}_{j, k}$ is the velocity of $j^{t h}$ spacecraft at time $k$.

By substituting Eq. (2.17) into Eq. (2.16), a closed form for $\tau$ can be obtained [69]:

$$
\tau=\frac{-B \pm \sqrt{B^{2}-4 A C}}{2 A}
$$


where,

$$
\begin{gathered}
A=\left(c^{2}-v_{j, k}^{2}\right) \\
B=2\left(\mathbf{r}_{i j, k}^{T} \mathbf{v}_{j, k}-r_{i j, k} v_{j, k}^{2} / c+c r_{i j, k}\right) \\
C=2 \mathbf{r}_{i j, k}^{T} \mathbf{v}_{j, k} r_{i j, k} / c-v_{j, k}^{2}\left(r_{i j, k} / c\right)^{2}
\end{gathered}
$$

where $v_{j, k}=\sqrt{\mathbf{v}_{j, k}^{T} \mathbf{v}_{j, k}}$, which is the magnitude of the $j^{\text {th }}$ spacecraft's velocity vector. Then, $T_{t r}$ can be computed using Eqs. (2.16) and (2.18). We assume that $t_{4}-t_{1}$ is small. Because $t_{1}$ is known, the position of $i^{t h}$ spacecraft, $\mathbf{r}_{i, k-3}$, at $t_{1}$ can be approximated as:

$$
\mathbf{r}_{i, k-3} \simeq \mathbf{r}_{i, k}-T_{41} \mathbf{v}_{i, k}
$$

where $T_{41}=t_{4}-t_{1}$.

The position of $j^{t h}$ spacecraft at time $t_{2}, \mathbf{r}_{j, k-2}$, can be approximated in a similar way:

$$
\mathbf{r}_{j, k-2} \simeq \mathbf{r}_{j, k}-\left(T_{t r}+T_{p r}\right) \times \mathbf{v}_{j, k}
$$

By substituting Eqs. (2.17), (2.22), and (2.23) into (2.15), the estimated TOA, $\hat{\mathbf{y}}_{\text {delay,TOA }}$ can be expressed in terms of the spacecraft position and velocity vectors at time $k$.

Similarly, in Figure 2.4, the time delay in signal transmission also impacts the DOA measurement [69]. However, the DOA measurement is defined as the angle of arrival of the signal transmitted by TRX to DBS. Therefore, the signal transmission and processing delay between $t_{1}$ and $t_{3}$ has no impact on DOA measurement. Here, a vector $\mathbf{L}$ and its components $X, Y$, and $Z$ are defined as:

$$
\begin{aligned}
\mathbf{L}=\left[\begin{array}{lll}
X & Y & Z
\end{array}\right]^{T} & =\mathbf{r}_{j, k-1}-\mathbf{r}_{i, k} \\
& =\mathbf{r}_{j, k}-T_{t r} \mathbf{v}_{j, k}-\mathbf{r}_{i, k}
\end{aligned}
$$

The measured DOA between the $i^{t h}$ and the $j^{t h}$ spacecraft for both relative azimuth and elevation angles $\bar{\theta}_{i j}$ and $\bar{\phi}_{i j}$ are:

$$
\tilde{\mathbf{y}}_{\text {delay }, D O A}=\left[\begin{array}{c}
\bar{\theta}_{i j} \\
\bar{\phi}_{i j}
\end{array}\right]+v_{\text {delay }, D O A}
$$


where,

$$
\begin{gathered}
\bar{\theta}_{i j}=\tan ^{-1} \frac{Y}{X} \\
\bar{\phi}_{i j}=\tan ^{-1} \frac{Z}{\sqrt{X^{2}+Y^{2}}}
\end{gathered}
$$

As in the case of the WLPS measurement model without the time delay effect (see Eqs. (2.12) and (2.13)), Eqs. (2.15) and (2.25) are nonlinear. Thus, the sensitivity matrix should be calculated to facilitate the process of linearization in the Extended Kalman Filter. The sensitivity matrix is derived below.

\subsubsection{Sensitivity matrix for TOA with Time Delay Model}

From equation (2.18), $\tau$ is a function of $\mathbf{r}_{i, k}, \mathbf{r}_{j, k}$ and $\mathbf{v}_{j, k}$. In this section, for simplicity, let $\mathbf{r}_{i}=\mathbf{r}_{i, k}, \mathbf{r}_{j}=\mathbf{r}_{j, k}, \mathbf{r}_{i j}=\mathbf{r}_{i j, k}$, and so on. Let:

$$
S_{r}=\left\|\mathbf{r}_{j, k-2}-\mathbf{r}_{i, k-3}\right\|
$$

The sensitivity matrix, $H_{\text {delay,TOA }}$, for equation (2.15) corresponds to:

$$
H_{\text {delay }, T O A}=\left[\begin{array}{llll}
\frac{\partial S_{r}}{\partial \mathbf{r}_{i}}+\frac{\partial r_{i j}}{\partial \mathbf{r}_{i}}+\frac{\partial \tau}{\partial \mathbf{r}_{i}} & \frac{\partial S_{r}}{\partial \mathbf{r}_{j}}+\frac{\partial r_{i j}}{\partial \mathbf{r}_{j}}+\frac{\partial \tau}{\partial \mathbf{r}_{j}} & \frac{\partial S_{r}}{\partial \mathbf{v}_{i}} & \frac{\partial S_{r}}{\partial \mathbf{v}_{j}}+\frac{\partial \tau}{\partial \mathbf{v}_{j}}
\end{array}\right]
$$

where,

$$
\begin{gathered}
\frac{\partial \tau}{\partial \mathbf{r}_{i}}=\frac{1}{A}\left[\frac{v_{j}^{2}}{c} \frac{\partial r_{i j}}{\partial \mathbf{r}_{i}}+\mathbf{v}_{j}^{T}+\frac{1}{D}\left\{-\mathbf{r}_{i j}^{T} \mathbf{v}_{j} \mathbf{v}_{j}^{T}+c^{2} r_{i j} \frac{\partial r_{i j}}{\partial \mathbf{r}_{i}}-v_{j}^{2} r_{i j} \frac{\partial r_{i j}}{\partial \mathbf{r}_{i}}\right\}\right] \\
\frac{\partial \tau}{\partial \mathbf{r}_{j}}=\frac{1}{A}\left[\frac{v_{j}^{2}}{c} \frac{\partial r_{i j}}{\partial \mathbf{r}_{j}}-c \frac{\partial r_{i j}}{\partial \mathbf{r}_{j}}-\mathbf{v}_{j}^{T}+\frac{1}{D}\left\{\mathbf{r}_{i j}^{T} \mathbf{v}_{j} \mathbf{v}_{j}^{T}+c^{2} r_{i j} \frac{\partial r_{i j}}{\partial \mathbf{r}_{i}}-v_{j}^{2} r_{i j} \frac{\partial r_{i j}}{\partial \mathbf{r}_{i}}\right\}\right] \\
\frac{\partial \tau}{\partial \mathbf{v}_{j}}=2 \frac{\tau}{A} \mathbf{v}_{j}^{T}+\frac{1}{A}\left[2 \frac{r_{i j}}{c} v_{j}^{T}-\mathbf{r}_{i j}^{T}+\frac{1}{D}\left\{\mathbf{r}_{i j}^{T} \mathbf{v}_{j} \mathbf{r}_{i j}^{T}-r_{i j}^{2} \mathbf{v}_{j}^{T}\right\}\right]
\end{gathered}
$$

The parameter $D$ in equations (2.30) to (2.32) is given by:

$$
D=\sqrt{\left(\mathbf{r}_{i j}^{T} \mathbf{v}_{j}\right)^{2}+c^{2} r_{i j}^{2}-r_{i j}^{2} v_{j}^{2}}
$$


And both $\frac{\partial r_{i j}}{\partial \mathbf{r}_{i}}$ and $\frac{\partial r_{i j}}{\partial \mathbf{r}_{j}}$ are given as:

$$
\frac{\partial r_{i j}}{\partial \mathbf{r}_{j}}=-\frac{\partial r_{i j}}{\partial \mathbf{r}_{i}} \equiv \frac{\mathbf{r}_{i j}^{T}}{r_{i j}}
$$

Finally, the partial derivatives of $S_{r}$ in Eq. (2.28) with respect to the absolute position and velocity vectors are given as:

$$
\begin{gathered}
\frac{\partial S_{r}}{\partial \mathbf{r}_{i}}=-\frac{1}{S_{r}}\left\{\left(\mathbf{r}_{j, k-2}-\mathbf{r}_{i, k-3}\right)^{T}+\left(\mathbf{r}_{j, k-2}-\mathbf{r}_{i, k-3}\right)^{T} \mathbf{v}_{j}\left[\frac{1}{c} \frac{\partial r_{i j}}{\partial \mathbf{r}_{i}}+\frac{\partial \tau}{\partial \mathbf{r}_{i}}\right]\right\} \\
\frac{\partial S_{r}}{\partial \mathbf{r}_{j}}=\frac{1}{S_{r}}\left\{\left(\mathbf{r}_{j, k-2}-\mathbf{r}_{i, k-3}\right)^{T}-\left(\mathbf{r}_{j, k-2}-\mathbf{r}_{i, k-3}\right)^{T} \mathbf{v}_{j}\left[\frac{1}{c} \frac{\partial r_{i j}}{\partial \mathbf{r}_{j}}+\frac{\partial \tau}{\partial \mathbf{r}_{j}}\right]\right\} \\
\frac{\partial S_{r}}{\partial \mathbf{v}_{i}}=\frac{T_{41}}{S_{r}}\left(\mathbf{r}_{j, k-2}-\mathbf{r}_{i, k-3}\right)^{T} \\
\frac{\partial S_{r}}{\partial \mathbf{v}_{j}}=-\frac{1}{S_{r}}\left\{\left(T_{t r}+T_{p r}\right)\left(\mathbf{r}_{j, k-2}-\mathbf{r}_{i, k-3}\right)^{T}+\left(\mathbf{r}_{j, k-2}-\mathbf{r}_{i, k-3}\right)^{T} \mathbf{v}_{j} \frac{\partial \tau}{\partial \mathbf{v}_{j}}\right\}
\end{gathered}
$$

\subsubsection{Sensitivity matrix for DOA with Time Delay Model}

The sensitivity matrix for DOA measurements can be derived in a similar way. The sensitivity matrix for the azimuth angle, $H_{\bar{\theta}}$, and elevation angle, $H_{\bar{\phi}}$, are:

$$
\begin{gathered}
H_{\bar{\theta}}=S_{\bar{\theta}}\left[\frac{1}{X} \frac{\partial Y}{\partial \mathbf{x}}-\frac{Y}{X^{2}} \frac{\partial X}{\partial \mathbf{x}}\right] \\
H_{\bar{\phi}}=S_{\bar{\phi}}\left[\frac{1}{\sqrt{X^{2}+Y^{2}}} \frac{\partial Z}{\partial \mathbf{x}}-\frac{Z}{\left(X^{2}+Y^{2}\right)^{3 / 2}}\left(X \frac{\partial X}{\partial \mathbf{x}}+Y \frac{\partial Y}{\partial \mathbf{x}}\right)\right]
\end{gathered}
$$

where,

$$
\begin{gathered}
S_{\bar{\theta}}=\frac{1}{1+\left(\frac{Y}{X}\right)^{2}} \\
S_{\bar{\phi}}=\frac{X^{2}+Y^{2}}{X^{2}+Y^{2}+Z^{2}}
\end{gathered}
$$

and,

$$
\begin{aligned}
& \frac{\partial \mathbf{L}}{\partial \mathbf{x}}=\left[\begin{array}{llll}
-I_{3 \times 3} & I_{3 \times 3} & \mathbf{0}_{3 \times 3} & -T_{t r} \times I_{3 \times 3}
\end{array}\right]-\mathbf{v}_{j, k} \frac{\partial T_{t r}}{\partial \mathbf{x}} \\
& \frac{\partial T_{t r}}{\partial \mathbf{x}}=\frac{1}{c} \frac{\partial r_{i j}}{\partial \mathbf{x}}+\frac{\partial \tau}{\partial \mathbf{x}}
\end{aligned}
$$


where, $\frac{\partial \mathbf{L}}{\partial \mathbf{x}}=\left[\begin{array}{lll}\frac{\partial X}{\partial \mathbf{x}}^{T} & \frac{\partial Y}{\partial \mathbf{x}} & \frac{\partial Z}{\partial \mathbf{x}}^{T}\end{array}\right]^{T}$.

Therefore, the time delay model for the relative position measurement between the $i^{t h}$ spacecraft and the $j^{\text {th }}$ spacecraft is given as:

$$
\tilde{\mathbf{y}}_{i j}=\left[\begin{array}{ll}
\tilde{\mathbf{y}}_{\text {delay }, T O A}^{T} & \tilde{\mathbf{y}}_{\text {delay }, D O A}^{T}
\end{array}\right]^{T}
$$

and the sensitivity matrix is

$$
H_{i j}=\left[\begin{array}{lll}
H_{\text {delay }, T O A}^{T} & H_{\bar{\theta}}^{T} & H_{\bar{\phi}}^{T}
\end{array}\right]^{T}
$$

\subsubsection{GPS Measurements Model}

In this section, the model of the GPS measurement is presented. We assume that there is no multipath effect and no clock bias error in the GPS receiver. For any GPS satellite that is in the $i^{t h}$ spacecraft's LOS, the pseudorange measurement is:

$$
\tilde{\rho}_{i}=\sqrt{\left(\mathbf{r}_{i}-\mathbf{r}_{G P S}\right)^{T}\left(\mathbf{r}_{i}-\mathbf{r}_{G P S}\right)}+v_{G P S}
$$

where $\mathbf{r}_{G P S}$ represents the position of the GPS satellite, and $v_{G P S}$ represents the GPS measurement noise which is assumed as zero mean white noise, with the noise covariance as $R_{G P S}=E\left\{v_{G P S} v_{G P S}^{T}\right\}$.

In reality, there might be more than four GPS satellites in the FOV of each spacecraft in the formation. However, in this chapter, we assume that only four GPS signals are observed at all times. Hence, the GPS measurement vector is:

$$
\tilde{\mathbf{y}}_{i, G P S}=\left[\begin{array}{llll}
\tilde{\rho}_{i, 1} & \tilde{\rho}_{i, 2} & \tilde{\rho}_{i, 3} & \tilde{\rho}_{i, 4}
\end{array}\right]^{T}
$$

The pseudorange measurements, $\tilde{\rho}_{1}$ to $\tilde{\rho}_{4}$ represent any four GPS signals received by the given spacecraft ( $i^{t h}$ spacecraft in this case). The corresponding sensitivity matrix, $H_{G P S}$, 
is

$$
H_{i, G P S}=\left[\begin{array}{cc}
\frac{\mathbf{r}_{i}-\mathbf{r}_{G P S, 1}}{\rho_{1}} & \mathbf{0}_{1 \times 3} \\
\frac{\mathbf{r}_{i}-\mathbf{r}_{G P S, 2}}{\rho_{2}} & \mathbf{0}_{1 \times 3} \\
\frac{\mathbf{r}_{i}-\mathbf{r}_{G P S, 3}}{\rho_{3}} & \mathbf{0}_{1 \times 3} \\
\frac{\mathbf{r}_{i}-\mathbf{r}_{G P S, 4}}{\rho_{4}} & \mathbf{0}_{1 \times 3}
\end{array}\right]
$$

Equation (2.49) shows the sensitivity matrix for a single spacecraft. However, it can be easily applied to spacecraft formation flying. For a GPS only scenario with $n$-spacecraft formation, the measurement vector is given as:

$$
\tilde{\mathbf{y}}=\left[\begin{array}{lll}
\tilde{\mathbf{y}}_{1, G P S}^{T} & \cdots & \tilde{\mathbf{y}}_{n, G P S}^{T}
\end{array}\right]^{T}
$$

with the sensitivity matrix, $H$, is

$$
H=\left[\begin{array}{c}
H_{1, G P S} \\
\vdots \\
H_{n, G P S}
\end{array}\right]
$$

For WLPS and GPS scenario, the measurement vector is given as:

$$
\tilde{\mathbf{y}}=\left[\begin{array}{lllllll}
\tilde{\mathbf{y}}_{1, G P S}^{T} & \ldots & \tilde{\mathbf{y}}_{n, G P S}^{T} & \tilde{\mathbf{y}}_{12}^{T} & \tilde{\mathbf{y}}_{13}^{T} & \ldots & \tilde{\mathbf{y}}_{i j}^{T}
\end{array}\right]^{T}
$$

with the sensitivity matrix, $H$, is

$$
H=\left[\begin{array}{llllll}
H_{1, G P S}^{T} & \ldots & H_{n, G P S}^{T} & H_{12}^{T} & H_{13}^{T} & \ldots H_{i j}^{T}
\end{array}\right]^{T}
$$

where $\tilde{\mathbf{y}}_{i j}$ and $H_{i j}$ are defined in Eqs. (2.45) and (2.46) respectively, $i=1, \ldots, n$, $j=1, \ldots, n$ and $i \neq j$.

\subsection{Extended Kalman Filter Implementation}

The EKF process begins with an initial estimated states, $\hat{\mathbf{x}}\left(t_{0}\right)=\hat{\mathbf{x}}_{0}$, and states covariance, $P\left(t_{0}\right)=P_{0}$. At every time step, $k$, a measurement is received from the sensor onboard, a gain matrix, $K$, is then computed as follows:

$$
K=P_{k}^{-} H_{k}^{T}\left[H_{k} P_{k}^{-} H_{k}^{T}+\mathscr{R}_{k}\right]^{-1}
$$


where $\mathscr{R}_{k}$ is the measurement noise covariance at step $k$, and its matrix composition depends on the availability of measurements (e.g. WLPS or GPS) in the estimation processes. The matrix $H_{k}$ is the sensitivity matrix, which is defined as:

$$
H_{k}=\left.\frac{\partial \hat{\mathbf{h}}}{\partial \mathbf{x}}\right|_{\hat{\mathbf{x}}_{k}^{-}}
$$

Here, the superscript “-” denotes predicted (or pre-update) estimates.

Then, the pre-update estimated states, $\hat{\mathbf{x}}_{k}^{-}$, and states covariance, $P_{k}^{-}$are updated through the following equations:

$$
\begin{gathered}
\hat{\mathbf{x}}_{k}^{+}=\hat{\mathbf{x}}_{k}^{-}+K\left[\tilde{\mathbf{y}}_{k}-\hat{\mathbf{h}}_{k}\left(\hat{\mathbf{x}}_{k}^{-}\right)\right] \\
P_{k}^{+}=\left(I-K H_{k}\right) P_{k}^{-}
\end{gathered}
$$

where $I$ is the identity matrix, $\hat{\mathbf{h}}_{k}\left(\hat{\mathbf{x}}_{k}^{-}\right)$is the estimated measurement, $\hat{\mathbf{x}}_{k}^{+}$is post-update estimated states, $P_{k}^{+}$is post-update states covariance, $K$ is the gain matrix in Eq. (2.54) and $H_{k}$ is the sensitivity matrix.

Both post-update estimated states, $\hat{\mathbf{x}}_{k}^{+}$, and states covariance, $P_{k}^{+}$are propagated to the next time step.

$$
\begin{gathered}
\dot{\hat{x}}=\mathbf{f}\left(t, \hat{\mathbf{x}}_{k}^{+}\right)+\mathbf{w} \\
\dot{P}=F_{k} P_{k}^{+}+P_{k}^{+} F_{k}^{T}+G Q G^{T}
\end{gathered}
$$

where, $\mathbf{w}$ is the process noise vector which is a zero mean gaussian noise with $Q=E\left\{\mathbf{w} \mathbf{w}^{T}\right\}, \mathbf{f}\left(t, \hat{\mathbf{x}}_{k}^{+}\right)$is obtained from the equation of motion of the spacecraft (Eq. (2.3)). $F_{k}$ is defined as:

$$
F_{k}=\left.\frac{\partial \mathbf{f}}{\partial \hat{\mathbf{x}}}\right|_{\hat{\mathbf{x}}_{k}^{+}}
$$

The $F_{k}$ matrix is the linearized state model:

$$
\Delta \dot{\mathbf{x}}=F_{k} \Delta \mathbf{x}+G \mathbf{w}
$$

where $\Delta \mathbf{x} \equiv\left[\begin{array}{llllll}\Delta \mathbf{r}_{1}^{T} & \ldots & \Delta \mathbf{r}_{n}^{T} \Delta \mathbf{v}_{1}^{T} & \ldots & \Delta \mathbf{v}_{n}^{T}\end{array}\right]^{T}$, with $\Delta \mathbf{r}_{i}$ and $\Delta \mathbf{v}_{i}$ are small changes in the $i^{t h}$ spacecraft position and velocity vectors. For $n$-spacecraft formation, the $F_{k}$ matrix in 
Eq. (2.61) is given by:

$$
F_{k}=\left[\begin{array}{ll}
\mathbf{0}_{3 n \times 3 n} & I_{3 n \times 3 n} \\
\left.\frac{d F(\mathbf{x})}{d \mathbf{x}}\right|_{\mathbf{x}=\hat{\mathbf{x}}_{k}^{+}} & \mathbf{0}_{3 n \times 3 n}
\end{array}\right]
$$

where,

$$
\begin{gathered}
\left.\frac{d F(\mathbf{x})}{d \mathbf{x}}\right|_{\mathbf{x}=\hat{\mathbf{x}}_{k}^{+}}=\left[\begin{array}{cccc}
\frac{\partial \mathbf{f}_{1}}{\partial \mathbf{x}_{1}} & \mathbf{0}_{3 \times 3} & \cdots & \mathbf{0}_{3 \times 3} \\
\mathbf{0}_{3 \times 3} & \frac{\partial \mathbf{f}_{2}}{\partial \mathbf{x}_{2}} & \cdots & \mathbf{0}_{3 \times 3} \\
\vdots & \ddots & \ddots & \vdots \\
\mathbf{0}_{3 \times 3} & \cdots & \cdots & \frac{\partial \mathbf{f}_{n}}{\partial \mathbf{x}_{n}}
\end{array}\right] \\
\frac{\partial \mathbf{f}_{i}}{\partial \mathbf{x}_{i}}=\frac{\mu}{s^{5}}\left[\begin{array}{cccc}
2 r_{i x}^{2}-r_{i y}^{2}-r_{i z}^{2} & 3 r_{i x} r_{i y} & 3 r_{i x} r_{i z} \\
3 r_{i x} r_{i y} & 2 r_{i y}^{2}-r_{i x}^{2}-r_{i z}^{2} & 3 r_{i y} r_{i z} \\
3 r_{i x} r_{i z} & 3 r_{i y} r_{i z} & 2 r_{i z}^{2}-r_{i x}^{2}-r_{i y}^{2}
\end{array}\right]
\end{gathered}
$$

where $i=1,2, \ldots, n, s=\sqrt{r_{i x}^{2}+r_{i y}^{2}+r_{i z}^{2}}$, and $\mathbf{r}_{i}=\left[\begin{array}{lll}r_{i x} & r_{i y} & r_{i z}\end{array}\right]^{T}$ in Eq. (2.5). Here, $\mu$ is defined as in Eq. (2.3).

For a configuration of $n$-spacecraft, the $G$ matrix, in Eq. (2.59) and Eq. (2.61) is:

$$
G \equiv\left[\begin{array}{c}
\mathbf{0}_{3 n \times 3 n} \\
I_{3 n \times 3 n}
\end{array}\right]
$$

The Kalman Filter algorithm for all scenarios is processed as follows. The estimated states (including both absolute position and velocity vectors) and the estimated state covariance are initialized. Then, the estimated state and state covariance are updated using Eqs. (2.56) and (2.57). It is noted that the sensitivity matrix and measurement vectors vary from one scenario to another. Then, both estimated states and state covariance are propagated using Eqs. (2.58) and (2.59) to next time step for future update.

\subsection{Simulation Results and Discussions}

Simulations are conducted to study and compare the estimation performance of the spacecraft position with respect to the Earth center (or absolute position) using WLPS 
only, GPS only, and WLPS plus GPS. The performance is assessed through computing the average of the Root Mean Square Error (RMSE), $\bar{\eta}$, of the estimated absolute positions of all spacecraft in the formation. The Mean Square Error (MSE) is the average of the square of estimation errors along the $x, y$, and $z$ axes of the spacecraft position.

$$
\eta_{i} \equiv \frac{\left\|\hat{\mathbf{r}}_{i}-\mathbf{r}_{i}\right\|}{\sqrt{3}}
$$

where $\eta_{i}$ is estimated $i^{t h}$ spacecraft's absolute position's RMSE, $\hat{\mathbf{r}}_{i}$ is the estimated absolute position of the $i^{t h}$ spacecraft and $\mathbf{r}_{i}$ is the truth absolute position of the $i^{t h}$ spacecraft.

For $n$-spacecraft formation, the $\bar{\eta}$ is given as:

$$
\bar{\eta}=\frac{1}{n} \sum_{i=1}^{n} \eta_{i}
$$

The convergence time, $T_{\text {conv }}$, of the estimation is defined as the time needed by the estimator until the RMSE falls below a given steady state threshold, $e_{t h r e s}$. The threshold varies with the SFF configuration (e.g., formation size, number of spacecraft). There are different approaches to determine the steady state threshold. In one of the approaches, $e_{\text {thres }}$ is computed by taking the average RMSE of the estimator at the steady state RMSE curve. However, the $T_{\text {conv }}$ is undefined if the RMSE does not converge.

Two case studies are presented in this chapter: (1) one examining the implementation of 1D WLPS for spacecraft navigation; and (2) comparing the estimation performance between a GPS standalone system, and a WLPS along with a GPS system with time delay modeling. In the second case study, the estimation performance is examined with respect to the following SFF configuration parameters: Size of formation, Measurement noise level, Number of spacecraft, and Altitude of the formation (except GPS scenario).

The general simulation assumptions in this chapter are: (i) Process and measurement noises for all spacecraft are zero-mean Gaussian; (ii) The attitude of each spacecraft is well-known; (iii) The TOA and DOA measurement noise standard deviations are 1 meter and 0.001 degrees respectively, unless otherwise specified; (iv) The GPS pseudo measurement noise standard deviation is 10 meters, unless otherwise specified; (v) The variance of the process noise is $10^{-14} \mathrm{~km}^{2} \mathrm{~s}^{-4}$; (vi) The initial estimated states covariance 
Table 2.1

Third and fourth spacecrafts' orbital elements.

\begin{tabular}{|c|c|c|c|c|}
\hline & Inclination (deg) & Arg. perigee (deg) & RAAN (deg) & Initial Anomaly (deg) \\
\hline S/C 1 & 5 & 0 & 5 & 3 \\
\hline S/C 2 & -5 & 0 & 0 & 2 \\
\hline S/C 3 & -3 & 0 & 7 & -4 \\
\hline S/C 4 & 3 & 0 & -4 & 10 \\
\hline S/C 5 & -2.5 & 0 & -3 & 2 \\
\hline S/C 6 & -10 & 0 & 0 & .5 \\
\hline
\end{tabular}

is $1 \mathrm{~km}^{2}$ along each position axis, and $0.01 \mathrm{~km}^{2} / \mathrm{s}^{2}$ along each velocity axis; and (vii) the simulation is run at the interval time of $\Delta t=10$ seconds for all scenarios.

In the simulations conducted in this chapter, we assume that each relative position in the formation is measured only once. Thus, for a two spacecraft formation, there is one relative measurement. Similarly, for three, four, and six spacecraft formation, there are three, six, and fifteen relative position measurements, respectively.

Figure 2.5 shows the accuracy performance of the EKF estimation using only the WLPS measurement with respect to different numbers of spacecraft in the formation. All the spacecraft have the same true semimajor axis of $7000 \mathrm{~km}$ and eccentricity of 0.05 . The orbital elements of all spacecraft are shown in Table 2.1. Figure 2.5 shows that the performance improves as the number of spacecraft in formation increases. The convergence time of the EKF process is about 400 minutes for the two-spacecraft formation. It is 300 minutes in the three-spacecraft formation, and is about 70 minutes for

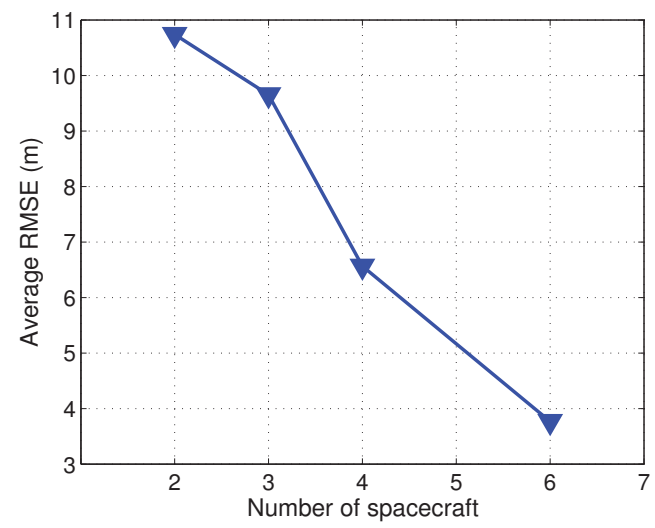

Figure 2.5: WLPS only - RMSE Performance. 
four spacecraft formations. The number of measurements in the three-spacecraft formation is three times higher than that of the two-spacecraft formation, and the number of measurements in the four-spacecraft formation is two times higher than that of the three-spacecraft formation. This leads to higher spacecraft orbit observability gain and results in significant convergence time improvement.

\subsubsection{One DOA measurement}

In this section, we compare the estimation performance between one DOA measurement and two DOA measurements. Both case studies include a TOA measurement. To study the feasibility of estimating the absolute positions from measurements of only the range and the azimuth angle, an observability analysis is needed. The EKF algorithm of 1-DOA is similar as the 2-DOA case. However, only the first two row of the sensitivity matrix in Eq. (2.12) is considered, because only one TOA and one DOA measurement available.

It is common that the observability analysis is conducted by linearizing the nonlinear problem. A numerical method for observability investigation is presented in detail in Ref. [5]. It can be shown that in this problem, the system is observable except in some special configurations of formations. One of those special cases is when two spacecraft are in the same circular orbit. In this section, we present simulation results for one observable case.

A two-spacecraft formation is considered. The spacecraft orbits have a semimajor axis of $7000 \mathrm{~km}$. The first spacecraft (observer) orbits in a circular orbit, with 0 degrees in Inclination, Argument of perigee, RAAN and initial true anomaly. The second spacecraft (target) orbits in an elliptic orbit with eccentricity of 0.05 degrees, inclination of 15 degrees, Argument of perigee of 0 degree, Right ascension of ascending node (RAAN) of 0 degree and initial true anomaly of 5 degrees.

We assume that only spacecraft 1 (S/C 1) is equipped with a DBS, while spacecraft 2 (S/C 2 ) is equipped with only a TRX. In this case study, the WLPS antenna array is rotated at 5 degrees about the body $\mathrm{x}$-axis, and both spacecraft experience no spinning motion. In this simulation, we assume the initial condition for both spacecraft is known and there is no signal transmission and signal processing time delay.

Figures 2.6(a) and 2.6(b) compare the RMSE performance between one DOA measurement and two DOA measurements, for different levels of measurements noises. 


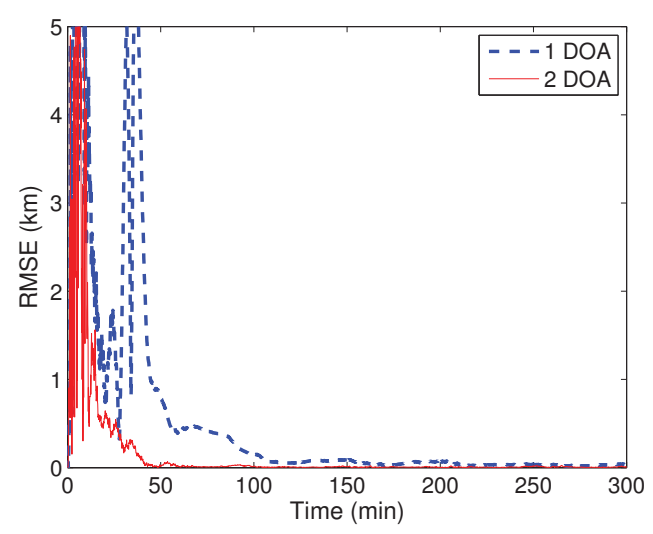

(a) Low TOA-DOA measurement noise.

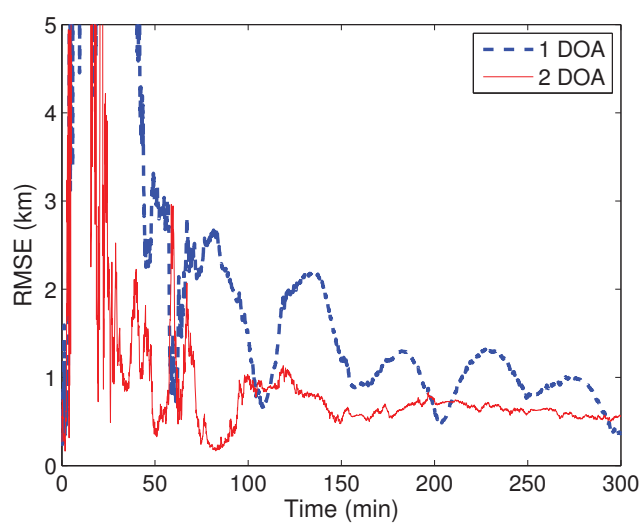

(b) High TOA-DOA measurement noise.

Figure 2.6: RMSE comparison of Azimuth only (1 DOA) versus Azimuth and Elevation (2 DOA) measurements.

The results indicate that it is possible to estimate the spacecraft absolute position with only one DOA measurement along with a TOA measurement, in the case presented.

Figure 2.6(b) compares the RMSE performance when both TOA and DOA noise levels are increased to 0.1 meter and 0.1 degrees respectively. The result shows that there is a significant difference between one DOA measurement performance and two DOA measurements performance. The two DOA measurements has a better overall accuracy compared to the one DOA measurement performance.

\subsubsection{GPS and WLPS versus GPS standalone system}

In this section, the performance of an orbit estimation algorithm using only GPS for position measurements is compared with the estimation algorithm that has an additional WLPS sensor (GPS/WLPS) installed on the spacecraft. We assume signal transmission time delay occurs between the DBS and TRX. Here, a four-spacecraft formation flying is considered. These comparisons will be carried out for several parameter ranges as detailed below. 
Table 2.2

Four Spacecraft Configuration for different formation size.

\begin{tabular}{|c|c|c|c|c|c|c|}
\hline Formation size & \multicolumn{2}{|c|}{$100 \mathrm{~km} / 200 \mathrm{~km}$} & \multicolumn{2}{|c|}{$700 \mathrm{~km} / 1400 \mathrm{~km}$} & \multicolumn{2}{|c|}{$1445 \mathrm{~km} / 2450 \mathrm{~km}$} \\
\hline & Min. & Max & Min. & Max & Min. & Max \\
\hline Inclination (deg) & -0.5 & 0.3 & -5 & 5 & -10 & 10 \\
\hline Arg. perigee (deg) & 0 & 0 & 0 & 0 & 0 & 0 \\
\hline RAAN (deg) & -0.5 & 0.2 & -4 & 7 & -3 & 3 \\
\hline True Anomaly (deg) & 0 & 0.5 & -4 & 10 & -2 & 5 \\
\hline
\end{tabular}

\subsubsection{Formation size}

First, the impact of formation size on the performance is studied. All spacecraft orbit at a semimajor axis of $7000 \mathrm{~km}$, with eccentricity of 0.05 . There are a total of four spacecraft in the formation. Here, ranges for the orbital elements of each spacecraft are listed in Table 2.2.

Table 2.3 compares the estimation performance with respect to different formation sizes. The results show that if only GPS measurement is available, the formation size does not impact the estimation performance in terms of accuracy. Because the GPS provides independent absolute position for each spacecraft in the formation, the relative spacing between spacecraft does not really affect the estimation accuracy, if GPS measurements only are used. In addition, the simulation results show that the convergence rate for all case studies is the same, which is 20 minutes. This is because the GPS measurement has more impact on the convergence rate of the estimation process than the WLPS measurement.

When the WLPS is implemented into the formation along with the GPS, the results show that the formation size impacts the estimation accuracy. Table 2.3 shows that as the

Table 2.3

Performance comparison between different formation size.

\begin{tabular}{|c|c|c|}
\hline Form. Size & Set up & Ave. RMSE $(\mathrm{m})$ \\
\hline \multirow{2}{*}{$100 \mathrm{~km} / 200 \mathrm{~km}$} & GPS/WLPS & 1.068 \\
& GPS & 2.114 \\
\hline \multirow{2}{*}{$700 \mathrm{~km} / 1400 \mathrm{~km}$} & GPS/WLPS. & 1.214 \\
& GPS & 2.087 \\
\hline \multirow{2}{*}{$1445 \mathrm{~km} / 2450 \mathrm{~km}$} & GPS/WLPS. & 1.384 \\
& GPS & 2.042 \\
\hline
\end{tabular}


Table 2.4

Performance comparison between high GPS noise and high WLPS noise.

\begin{tabular}{|c|c|c|c|}
\hline Noise level & Set up & Ave. RMSE (m) & Conv. time (min) \\
\hline High GPS & GPS/WLPS & 9.529 & 70 \\
Noise & GPS & 62.745 & 70 \\
\hline High WLPS & GPS/WLPS & 1.669 & 20 \\
Noise & GPS & 2.090 & 20 \\
\hline
\end{tabular}

formation size decreases, the estimation accuracy increases. Although the improvement may seem insignificant (only 30 decimeters) when the WLPS is implemented together with GPS, there is about $25 \%$ improvement when the formation size decreases from $1445 \mathrm{~km} / 2450 \mathrm{~km}$ to $100 \mathrm{~km} / 200 \mathrm{~km}$. For other cases where the RMSE is higher, the impact may become more significant.

\subsubsection{Measurement noise level}

Next, consider the $700 \mathrm{~km} / 1400 \mathrm{~km}$ formation size configuration shown in Table 2.2. The measurement noises of WLPS and GPS are varied in the following order: a. High GPS noise: Both TOA and DOA noises levels are $0.001 \mathrm{~km}$ and 0.001 degrees respectively, and GPS noise level is $1 \mathrm{~km}$; and, b. High WLPS noise: Both TOA and DOA noises levels are $0.01 \mathrm{~km}$ and 0.01 degrees respectively, and GPS noise level is $0.01 \mathrm{~km}$.

Table 2.4 compares the RMSE and convergence rate with respect to two different noise levels, high GPS noise level and high WLPS noise level. With high GPS noise (such as signal jamming), the result shows that we are still able to achieve good estimation accuracy (up to meters accuracy) with the aid of WLPS measurements. Besides that, additioinal WLPS measurement is capable of improving the estimation performance if compared to the GPS standalone system; even though the WLPS measurement noise level is high. In addition, the convergence rate is increased if GPS measurement noise increased. This result confirms that the GPS has more impact on the convergence rate of estimation. 


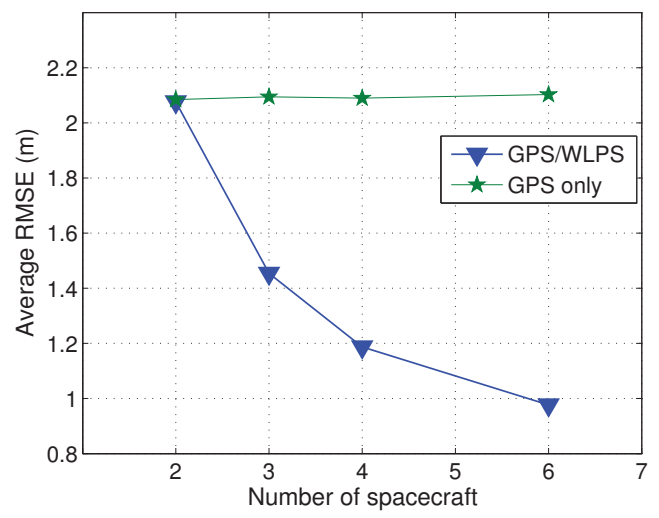

(a) RMSE comparison with respect to number of spacecraft.
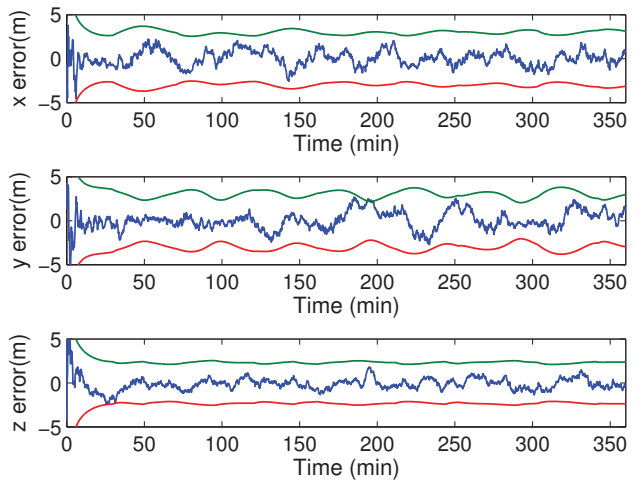

(b) GPS/WLPS position error with three sigma boundaries.

Figure 2.7: Position RMSE comparison of GPS-only and GPS/WLPS, and three sigma boundaries plot.

\subsubsection{Number of spacecraft in formation}

The impact of different number of spacecraft on the estimation performance is studied. Four formations are considered: two, three, four, and six spacecraft. The size of the formation in the four cases is $700 \mathrm{~km} / 1400 \mathrm{~km}$.

Figure 2.7(a) compares the accuracy and convergence performance with respect to the number of spacecraft in the formation. Because GPS offers absolute position measurements for each spacecraft, independent from other spacecraft in the formation, increasing the number of spacecraft in the formation does not affect the estimation accuracy, if no WLPS is used. Figure 2.7(a) shows that as the number of spacecraft increases, the RMSE performance improves. It is noted that we assume spacecraft $i$ measures spacecraft $j$ 's relative position, but spacecraft $j$ does not measure spacecraft $i$ 's relative position in the simulation.

Figure 2.7(b) shows the spacecraft's estimated absolute position error with a three sigma boundary for the WLPS and GPS scenario. The simulation is processed for 100 Monte Carlo runs with the initial condition error of $1 \mathrm{~km}$ in absolute position vector and $1 \mathrm{~ms}^{-1}$ in absolute velocity vector for each spacecraft in the formation. The initial state covariance remains the same as presented in the simulation assumption. Figure 2.7(b) shows that the estimated absolute position error falls within the three sigma boundary. The estimated state error converges at the same pace as the state covariance in the presence of GPS 
measurements. 


\section{Chapter 3}

\section{Implementation of a Differential Geometric Filter For Spacecraft Formation Orbit Estimation}




\subsection{Introduction}

The relative and absolute positions estimation of spacecraft formations is a fundamental task in many space missions.* Relative position estimation plays an important role in Spacecraft Formation Flying (SFF) missions, a subject that has been the focus of much research during last decade. Some SFF missions require that multiple spacecraft, in different orbits, communicate without interruption, e.g., satellites in the CITRIS-COSMIC system are required to communicate with each other to monitor the ionospheric irregularities [1]. Relative positions between satellites, such as Cluster and Cluster-II satellites launched by the European Space Agency, are estimated and controlled to support many collaborative tasks where satellites are required to maintain a specific formation in a continuous manner within the mission period [2]. Several relative position estimation methods have been developed. A GPS-like technology that can be applied to SFF has been introduced [3,4], where each spacecraft is equipped with a communication system to localize other spacecraft in the formation. This system provides high precision estimates for relative positions. Yet, complex hardware is required on each spacecraft. This system does not stand alone and requires a GPS system.

A relative position and attitude estimation through a Vision-Based Navigation System (VISNAV) has been addressed extensively in the literature [7, 49-51]. The VISNAV consists of a Position Sensing Diode (PSD) sensor and an array of LED source beacons installed on a plate. The PSD detects the energy of the light source emitted by the beacons. Then the PSD generates a current flow to four terminals that are installed on the PSD plate. At that point, the centroid of the current flow is determined to measure the direction of the energy source. The covariance study shows that the performance could be affected by the relative distance between spacecraft. The accuracy factors of the estimation depend on the number of beacons installed on the spacecraft. Three or more beacons are suggested to ensure the observability of the system [7].

Mark Psiaki proved the feasibility of estimating the orbits of two spacecraft through measuring their relative distance and azimuth and elevation angles [5]. The orbital elements estimation accuracy depends on the relative distances between the spacecraft. Only two spacecraft formations were considered. As shown in Ref. [5], the relative position between spacecraft impacts the estimation performance. In addition, the system

*The material contained in this chapter was previously published in the International Journal of Aerospace Engineering. 
becomes unobservable in a few cases, such as zero inclination.

The Extended Kalman Filter (EKF) has been widely implemented into the nonlinear system to estimate the relative and absolute positions in SFF [7, 16, 78, 79]. The system nonlinearity causes the EKF estimation to be sensitive to initial condition errors, and the linearization step in the EKF may result in estimation instability. The Unscented Kalman Filter (UKF) has been implemented for relative attitude and position estimation in SFF $[17,18]$. The UKF has a better robustness to initial condition errors compared to the EKF. However, its computational complexity is higher than the standard EKF. If compared to the standard EKF, the UKF has a faster convergence rate, but the computational time required for each update is longer than that of the EKF [80]. The Batch filter [22], an offline filtering method, has been implemented in Ref. [5] for absolute positions estimation using the relative positions measurements. Both studies in [16] and [5] show that the configuration of the SFF affects the stability and the accuracy of estimation.

The Differential Geometric (DG) Theory has been widely implemented in the control and guidance research area [42, 43]. In calculus, the Differential Geometry represents the linear approximation of a smooth curve [44]. Ref. [45] and [46] introduced the Differential Geometric approach into the missile tracking applications. In Ref. [45], the system model is transformed from the arc length domain into the time domain; then the guidance law is applied. Both papers have shown the feasibility of implementing the DG for missile guidance.

The Differential Geometric approach for nonlinear systems has been extended recently for the nonlinear estimation purpose [47]. The DG estimation avoids the linearization step in the EKF. In DG estimation, the nonlinear dynamics are mapped to a linear domain, where a linear estimator can be implemented. Then, the inverse transformation is applied to the estimator [47]. Ref. [47] details how the DG estimation can be implemented when the number of available measurements are too few to handle all the system nonlinearities, and shows that both the optimal control and the linear filtering approaches can be applied in the mapped linear domain.

In this chapter, the DG filter is implemented in estimating the spacecraft relative and absolute positions in formation. While most of the developments in the literature focus on the estimation of relative positions [3, 7], this chapter estimates relative and absolute positions of all spacecraft. The Differential Geometric (DG) Estimation and the Extended Kalman Filter (EKF) are implemented and compared for estimation using Wireless Local 
Positioning System (WLPS) measurements. The WLPS installed on each spacecraft enables that spacecraft to determine the relative positions of other spacecraft located in its coverage area via Time-of-Arrival (TOA) and Directional-of-Arrival (DOA) measurements $[16,58,81]$.

In this chapter, two scenarios are considered: (1) observations include WLPS measurements only, and (2) observations include WLPS measurements in addition to the absolute position of one spacecraft measured by radar systems installed on the earth. Section 3.2 presents the radar measurement model. The derivation of the DG Estimation equations for the problem of relative and absolute positions estimation are presented in Section 3.3. Section 3.4 presents the EKF implementation. Section 3.5 discusses the simulation results and presents a complexity analysis that compares the computational costs between the DG filter and the EKF.

\subsection{Measurement Model}

In this section, the measurement models for each scenario are derived. Two sets of models are derived for two scenarios: (1) Only WLPS measurements are available, and (2) WLPS measurements and those taken by a tracking system (such as a radar installed on the ground) are available. For the first scenario, a four-spacecraft formation is considered. Each spacecraft is equipped with both a DBS and a TRX. For the second scenario, a two-spacecraft formation is considered; one spacecraft is equipped with a DBS and the other spacecraft is equipped with a TRX. The radar measures the absolute range and the absolute azimuth and elevation angles of one spacecraft with respect to a ground station. Since the position of the ground station is known, we assume the absolute position measurement is expressed with respect to earth center [82].

The estimated states are considered to be the spacecrafts' absolute and relative positions, and their velocities. If the spacecrafts' orientations are known, the WLPS measurements can be expressed in the inertial reference frame. 


\subsubsection{WLPS Measurements Model}

The WLPS measurement model without the time delay transmission has been derived in Section 2.3.2. Using (2.8) and (2.9), we can express the WLPS measurement between the $i^{\text {th }}$ and the $j^{\text {th }}$ spacecraft as:

$$
\tilde{\mathbf{y}}_{p, i j}=\left[\begin{array}{l}
h_{i j}^{T O A} \\
\mathbf{h}_{i j}^{D O A}
\end{array}\right]+v_{p, i j}
$$

where $v_{p, i j}$ denotes WLPS measurement noise, which is assumed to be zero-mean Gaussian with $E\left[v_{p, i j} v_{p, i j}^{T}\right]=\Re_{p, i j}$.

Using (8.4) - (8.6) in Appendix 8.1, the WLPS measurement vector in Cartesian coordinates is:

$$
\tilde{\mathbf{y}}_{c, i j}=\left[\begin{array}{l}
r_{i j, x} \\
r_{i j, y} \\
r_{i j, z}
\end{array}\right]+v_{c, i j}
$$

The new measurement noise $v_{c, i j}$ is a function of $v_{p, i j}, h_{i j}^{T O A}$ and $\mathbf{h}_{i j}^{T O D}$ defined in (3.1).

\subsubsection{Radar System Model}

In the second scenario, an additional radar system that measures the spacecraft range, azimuth and elevation angles is included. It is assumed that the $i^{\text {th }}$ spacecraft that is observed by the radar is also equipped with a DBS. The absolute position measurement made by the radar is:

$$
\tilde{\mathbf{y}}_{r, i}=\left[\begin{array}{lll}
\rho_{i} & \lambda_{r_{i}} & \xi_{r_{i}}
\end{array}\right]^{T}+v_{r, i}
$$

where $v_{r, i}$ is radar system noise, which is assumed to be zero-mean Gaussian, and $E\left[v_{r} v_{r}^{T}\right]=\Re_{r}$. The $r_{i}$ is the absolute distance of the $i^{t h}$ spacecraft from the radar, $\lambda_{r_{i}}$ is the azimuth angle and $\xi_{r_{i}}$ is the elevation angle of $i^{\text {th }}$ spacecraft with respect to a radar-fixed coordinate system. The radar position is known in the ECI frame. So, using radar measurements, it is straightforward to calculate the absolute distance of the $i^{t h}$ spacecraft from the earth's center, $\left\|\mathbf{r}_{i}\right\|$, and the azimuth, $\lambda_{i}$, and elevation, $\xi_{i}$, angles with respect to the ECI frame. In this preliminary analysis, we will assume for simplicity that we measure directly $\left\|\mathbf{r}_{i}\right\|, \lambda_{i}$, and $\xi_{i}$. Azimuth and elevation angles are related to the coordinates of the 
spacecraft through:

$$
\begin{gathered}
\lambda_{i}=\tan ^{-1} \frac{r_{i, y}}{r_{i, x}} \\
\xi_{i}=\tan ^{-1} \frac{r_{i, z}}{\sqrt{r_{i, x}^{2}+r_{i, y}^{2}}}
\end{gathered}
$$

where $r_{i, x}, r_{i, y}$ and $r_{i, z}$ are defined in (2.5).

\subsubsection{Measurement Models for Simulation}

In the first scenario, only the linear filtering method presented in Section 3.3 will be implemented. Four-spacecraft formation is required, as discussed in Section 3.3.3. Thus, the measurement vector, $\tilde{\mathbf{y}}$ is:

$$
\tilde{\mathbf{y}} \equiv\left[\begin{array}{llll}
\tilde{\mathbf{y}}_{c, 12}^{T} & \tilde{\mathbf{y}}_{c, 23}^{T} & \tilde{\mathbf{y}}_{c, 34}^{T} & \tilde{\mathbf{y}}_{c, 41}^{T}
\end{array}\right]^{T}+v
$$

where $v \equiv\left[\begin{array}{llll}v_{c, 12}^{T} & v_{c, 23}^{T} & v_{c, 34}^{T} & v_{c, 41}^{T}\end{array}\right]^{T}$ is the measurement noise vector. The corresponding measurement noise covariance is given as:

$$
\mathfrak{R}_{1}=\left[\begin{array}{llll}
\mathfrak{R}_{c, 12} & \mathbf{0}_{3 \times 3} & \mathbf{0}_{3 \times 3} & \mathbf{0}_{3 \times 3} \\
\mathbf{0}_{3 \times 3} & \mathfrak{R}_{c, 23} & \mathbf{0}_{3 \times 3} & \mathbf{0}_{3 \times 3} \\
\mathbf{0}_{3 \times 3} & \mathbf{0}_{3 \times 3} & \mathfrak{R}_{c, 34} & \mathbf{0}_{3 \times 3} \\
\mathbf{0}_{3 \times 3} & \mathbf{0}_{3 \times 3} & \mathbf{0}_{3 \times 3} & \mathfrak{R}_{c, 41}
\end{array}\right]
$$

The $\mathfrak{R}_{c, i j}$ which denotes the relative position measurement noise covariance in the ECI frame in equation (3.7) is given as:

$$
\begin{aligned}
\mathfrak{R}_{c, i j} & =E\left\{\left(\mathbf{r}_{i j}-D_{i}^{T} v_{c, i j}\right)\left(\mathbf{r}_{i j}-D_{i}^{T} v_{c, i j}\right)^{T}\right\} \\
& =D_{i}^{T} \bar{\Re}_{c, i j} D_{i}
\end{aligned}
$$

where $\bar{\Re}_{c, i j}$ is the noise covariance of the relative position measurement, expressed in cartesian coordinates, and $D_{i}$ is the attitude matrix [22]. $\Re$ is given as a function of $\Re_{p, i j}$ [82]. The transformation from $\Re_{p, i j}$ to $\bar{\Re}$ is shown in Appendix section. 
For the second scenario, both the linear filtering method and pole placement, which are presented in Section 3.3, will be implemented for estimation purposes. Only two-spacecraft formation is considered. The measurement vector $\tilde{\mathbf{y}}$ is:

$$
\tilde{\mathbf{y}} \equiv\left[\begin{array}{ll}
\tilde{\mathbf{y}}_{c, 12}^{T} & \tilde{\mathbf{y}}_{r, 1}^{T}
\end{array}\right]^{T}+v
$$

where $v \equiv\left[\begin{array}{ll}v_{c, 12}^{T} & v_{r, 1}^{T}\end{array}\right]^{T}$. The corresponding measurement covariance matrix $\Re_{2}$ is:

$$
\mathfrak{R}_{2}=\left[\begin{array}{cc}
\mathfrak{R}_{12} & \mathbf{0}_{3 \times 3} \\
\mathbf{0}_{3 \times 3} & \mathfrak{R}_{r}
\end{array}\right]
$$

where $\mathfrak{R}_{r}=\operatorname{diag}\left[\begin{array}{lll}\sigma_{\rho}^{2} & \sigma_{\lambda}^{2} & \sigma_{\xi}^{2}\end{array}\right]$, which the "diag" represents the diagonal matrix, and $\sigma_{\rho}^{2}$, $\sigma_{\lambda}^{2}$ and $\sigma_{\xi}^{2}$ are the measurement noise variances for absolute distance, azimuth, and elevation angle, respectively.

\subsection{Differential Geometric Filter}

DG estimation was recently extended to deal with nonlinear dynamic systems with fewer measurements than required to handle all nonlinearities [47]. DG estimation avoids the linearization step encountered in the EKF. In the DG estimation, the nonlinear system is transformed into a linear system. The transformation requires the state vector, $\mathbf{x}$, to be expressed as a function of the measurements vector, $\mathbf{y}$, i.e., $\mathbf{x}=s(\mathbf{y})$.

The DG estimation development is explained in Ref. [47] and is briefed here for completness of presentation. The system model for DG estimation is constructed by defining a new state vector $\mathbf{z}$ such that the measurement vector $\mathbf{y}$ can be expressed as a linear function of $\mathbf{z}$ :

$$
\begin{gathered}
\dot{\mathbf{z}}=A \mathbf{z}+B g(\mathbf{z})+G \mathbf{w} \\
\mathbf{y}=C \mathbf{z}+v
\end{gathered}
$$

Here, $\mathrm{A}, \mathrm{B}, \mathrm{C}$, and $\mathrm{G}$ are linear matrices, $\mathbf{w}$ is the process noise, and $v$ is the measurement noise. The state equation (3.11) includes a linear system, $A \mathbf{z}$, and a nonlinear input function, $B g(\mathbf{z})$. 
When the available measurements are not enough to handle the nonlinearities in the system, a set of pseudo-measurements, $\mathbf{z}^{\prime}$, is required. The pseudo-measurements can be obtained by taking the derivatives of $\mathbf{y}$, i.e., $\mathbf{z}^{\prime}=\dot{\mathbf{y}}$.

The pseudo-errors are defined as:

$$
\mathbf{e}=\mathbf{z}^{\prime}-\hat{\mathbf{z}}^{\prime}
$$

Then, the estimated state vector, $\hat{\mathbf{z}}$, that includes the pseudo-measurements is:

$$
\hat{\mathbf{z}} \equiv\left[\begin{array}{cc}
\hat{\mathbf{y}}^{T} & \dot{\hat{\mathbf{y}}}^{T}
\end{array}\right]^{T}=\left[\begin{array}{ll}
\hat{\mathbf{y}}^{T} & \mathbf{z}^{T}
\end{array}\right]^{T}
$$

Letting $m$ be the total number of measurements, Eq. (3.14) shows that the size of the estimated state vector, $\mathbf{z}$, is twice the size of the measurement vector, $n=2 m$. In addition, it can be shown that the time rate of change of the pseudo-errors are [47]:

$$
\hat{\dot{\mathbf{e}}}=A_{m+1, n} \hat{\mathbf{e}}^{-}-L_{m+1, n}(\tilde{\mathbf{y}}-\hat{\mathbf{y}})
$$

where $L$ is the gain matrix which will be presented in the next section, and $A_{m, n}$ and $L_{m, n}$ denotes $m^{\text {th }}$ to $n^{\text {th }}$ row of the $A$ and $L$ matrices respectively.

In Ref. [47], two gain computation methods are presented, which are the pole placement method and Kalman Filter (or Linear Filtering) method.

\subsubsection{Linear Filtering Gain}

The state equations (3.11) are linear. Thus, the Kalman Filter theory can be applied. Note that the input function, $g(\mathbf{z})$, is not used in the updating process in both DG estimation and EKF. The DG estimation gain matrix, $L$, is determined by:

$$
L=P C^{T}\left(C P C^{T}+R\right)^{-1}
$$

where $P$ is the state covariance matrix corresponding to the $\mathbf{z}$ vector, and $R$ is the measurement noise covariance matrix. Then, the state estimates, $\mathbf{z}$, and its covariance are 
updated as follows:

$$
\begin{gathered}
\hat{\mathbf{z}}^{+}=\hat{\mathbf{z}}^{-}+L_{1, m}(\tilde{\mathbf{y}}-\hat{\mathbf{y}}) \\
\hat{\mathbf{z}}^{\prime+}=\hat{\mathbf{z}}^{\prime-}+L_{m+1, n}(\tilde{\mathbf{y}}-\hat{\mathbf{y}}) \\
P^{+}=(I-L C) P^{-}
\end{gathered}
$$

where $I$ is the identity matrix, the superscript - denotes pre-update estimates and the superscript + denotes post-update estimates. The $\hat{\mathbf{z}}^{\prime}$ denotes any estimated pseudo-measurements vectors and the estimated state vectors that are not observed by measurements. $L_{m, n}$ denotes $m^{\text {th }}$ to $n^{\text {th }}$ row of the gain matrix, $L$.

The DG estimation filtering process works as follows [47]. The gain matrix, $L$, is determined using (3.16). Then the estimated states and state covariance matrix are updated using (3.17) to (3.19). The updated states and the pseudo-errors are propagated by (3.11) and (3.15) with their respective input function. The state covariance matrix is propagated using the equation (3.20):

$$
\dot{P}=A P+P A^{T}+G Q G^{T}
$$

where $Q$ is the process noise covariance, which is defined as $Q=E\left\{\mathbf{w} \mathbf{w}^{T}\right\}$.

Both the $A$ and $G$ matrices in (3.11) and (3.20) are linear time invariant matrices; thus, (3.20) can be expresed in time discrete representation, which is given as:

$$
P_{k+1}=\Phi P_{k} \Phi^{T}+\mathscr{Q}
$$

where, subscript $k$ and $k+1$ denote the current and next time step respectively, $\Phi$ is the state transition matrix and $\mathscr{Q}$ is the discrete-time process noise covariance. Both $\Phi$ and $\mathscr{Q}$ are calculated as follows [83]. Let,

$$
\begin{gathered}
\mathscr{A}=\left[\begin{array}{cc}
-A & G Q G^{T} \\
\mathbf{0} & F^{T}
\end{array}\right] \Delta t \\
\mathscr{B}=e^{\mathscr{A}} \equiv\left[\begin{array}{cc}
\mathscr{B}_{11} & \mathscr{B}_{12} \\
\mathbf{0} & \mathscr{B}_{22}
\end{array}\right]
\end{gathered}
$$

where $\Delta t$ is the time interval between two successive measurements, $e^{\mathscr{A}}$ is the exponential 
matrix of $\mathscr{A}$ matrix, and $F$ is the matrix representation of the system dynamic model. For the nonlinear dynamic model case, e.g., Eq. (2.3), $F$ is the first order Taylor series expansion (or Jacobian matrix) of the dynamic model. Both $\Phi$ and $\mathscr{Q}$ are given as:

$$
\begin{gathered}
\Phi=\mathscr{B}_{22}^{T} \\
\mathscr{Q}=\Phi \mathscr{B}_{12}
\end{gathered}
$$

\subsubsection{Pole Placement Method}

Another filtering method presented in Ref. [47] is the pole placement method. The pole placement method has been widely used in controls and estimation. Unlike the Kalman Filter, the gain, $L$, in pole placement, does not change dynamically. Pole placement also requires full system observability. However, the pole placement method guarantees the linearity of the measurement model, and hence guarantees stability.

The pole placement gain matrix $L$ is computed as follows. Given that the $A$ matrix has $n \times n$ dimensional and canonical form:

$$
A=\left[\begin{array}{ccccc}
1 & 0 & 0 & \ldots & 0 \\
0 & 1 & 0 & \ldots & 0 \\
0 & 0 & 1 & \ldots & 0 \\
\vdots & \vdots & \vdots & \ddots & \vdots \\
0 & 0 & 0 & \ldots & 0
\end{array}\right]
$$

and $C$ matrix has $n \times m$ dimensional. The system is considered to be fully observable if the rank of $O(C, A)$ matrix is full rank [84]:

$$
O(C, A)=\left[\begin{array}{c}
C \\
C A \\
C A^{2} \\
\vdots \\
C A^{n-1}
\end{array}\right]
$$

If the full rank observability condition in equation (3.27) is fulfilled, the gain matrix $L$ is 
calculated using the following equation:

$$
\lambda=\operatorname{eig}(A-C L)
$$

where $\lambda$ is the eigenvalue vector, and eig represents the eigenvalue.

If a set of eigenvalues is given, the gain matrix $L$ can be computed using the Ackermann's formula [22]. The selection of the eigenvalues impacts both stability and accuracy of

estimation. Therefore, negative eigenvalues are always selected to ensure the stability of the estimation process.

Here, our goal is to set up the DG estimation filter for the two scenarios introduced in Section III. For the first scenario, only the WLPS measurement is available while we intend to estimate the spacecraft absolute position. Thus, it is required to derive the expression of absolute position of spacecraft in terms of relative position. For the second scenario, an additional absolute position measurement of spacecraft is included.

\subsubsection{Scenario One - Relative Position Estimation with WLPS-only measurement.}

The absolute distance of a spacecraft $j$ from the Earth's center is:

$$
r_{j}^{2}=r_{i}^{2}+r_{i j}^{2}+2 \mathbf{r}_{i j}^{T} \mathbf{r}_{i}
$$

where $\mathbf{r}_{i}$ and $\mathbf{r}_{i j}$ denote absolute and relative positions respectively defined in (2.4) to (2.6).

The orbital equation of spacecraft is [75]:

$$
r=\frac{a\left(1-\varepsilon^{2}\right)}{1+\varepsilon \cos v}
$$

In (3.30), $r$ represents the distance of spacecraft to the Earth's center, $a$ is the semimajor axis, $\varepsilon$ is the eccentricty, and $v$ is the true anomaly. In this scenario, it is assumed that all spacecraft in the formation have the same semimajor axis, eccentricity, and true anomaly. Hence, Eq. (3.30) shows that the distances from all spacecraft to the Earth's center would 
be the same. In this case, $r_{j}=r_{i}$, leads to:

$$
r_{i j}^{2}+2 \mathbf{r}_{i j}^{T} \mathbf{r}_{i}=0
$$

If we consider a four-spacecraft configuration, then (3.31) can provide a closed form solution for absolute positions using relative position measurements. For example, the absolute position of spacecraft 1 can be obtained from:

$$
\left[\begin{array}{lll}
r_{12, x} & r_{12, y} & r_{12, z} \\
r_{13, x} & r_{13, y} & r_{13, z} \\
r_{14, x} & r_{14, y} & r_{14, z}
\end{array}\right]\left[\begin{array}{c}
r_{1, x} \\
r_{1, y} \\
r_{1, z}
\end{array}\right]=-\left[\begin{array}{c}
\frac{r_{12}^{2}}{2} \\
\frac{r_{13}^{2}}{2} \\
\frac{r_{14}^{2}}{2}
\end{array}\right]
$$

Here, $r_{i, x}, r_{i, y}, r_{i, z}$ and $r_{i j, x}, r_{i j, y}, r_{i j, z}$ are the absolute and relative position elements introduced in (2.4), respectively. The right hand side of (3.32) are the square of relative distances between the spacecraft. Similarily, we can determine the absolute positions of the other spacecraft. Note that the solution in (3.32) requires the computation of inverse of the relative position measurements matrix. Therefore, the closed form solution needs an accurate estimation or unbiased relative position measurements.

Consider the four sets of WLPS measurements mentioned in Section III where the total number of measurements, $m$, in (3.6) is 12 . However, there are 24 states to be estimated; thus, at least 12 pseudo-measurements are required. The pseudo-measurements are defined as the relative velocities. Using the dynamic motion of spacecraft defined in (2.3), the relative accelerations between spacecraft are:

$$
\ddot{\mathbf{r}}_{i j}=\frac{\mu \mathbf{r}_{i}}{r_{i}^{3}}-\frac{\mu \mathbf{r}_{j}}{r_{j}^{3}}
$$

When all spacecraft in the formation have the same semimajor axis, $a$, eccentricity, $\varepsilon$, and true anomaly, $v$, at all times, (3.33) can be simplified to:

$$
\ddot{\mathbf{r}}_{i j}=-\frac{\mu \mathbf{r}_{i j}}{r^{3}}
$$

The nonlinear input function, $g(\mathbf{z})$, in (3.11) is defined as a function of measurements, $\tilde{\mathbf{y}}$, and pseudo measurement, $\mathbf{z}^{\prime}$. The only nonlinear function of the dynamic system in this scenario is the relative acceleration introduced in (3.34). Therefore, the input function 
consists of the relative acceleration between the spacecraft, which is:

$$
g\left(\tilde{\mathbf{y}}, \mathbf{z}^{\prime}\right)=\left[\begin{array}{llll}
-\frac{\mu \mathbf{r}_{12}^{T}}{r^{3}} & -\frac{\mu \mathbf{r}_{23}^{T}}{r^{3}} & -\frac{\mu \mathbf{r}_{34}^{T}}{r^{3}} & -\frac{\mu \mathbf{r}_{41}^{T}}{r^{3}}
\end{array}\right]^{T}
$$

where $\mathbf{z}^{\prime}$ is the pseudo-measurements vector defined in Eq. (3.13), which can be written as $\mathbf{z}^{\prime}=\hat{\mathbf{z}}^{\prime}+\mathbf{e}$.

Equation (3.35) depicts that the absolute range of spacecraft is required for the relative dynamic model. Equation (3.32) determines the absolute position using relative position measurements. However, (3.32) is vulnerable to the error in $\mathbf{r}_{i j}$, which could result in a large estimation error in the absolute range, $r$. Then, we consider two additional pseudo-measurements which are the absolute range and eccentric anomaly, $E$. The rate of change of absolute range is [75]:

$$
\dot{r}=a \varepsilon \sin E \dot{E}
$$

where $a$ and $\varepsilon$ were defined in (3.30). The rate of change of eccentric anomaly is:

$$
\dot{E}=\sqrt{\frac{\mu}{a}} \frac{1}{r}
$$

Substituting (3.37) into (3.36), we obtain the expression of $\dot{r}$ in terms of eccentric anomaly and range only. Adding (3.36) and (3.37) into the input function, $g\left(\tilde{\mathbf{y}}, \mathbf{z}^{\prime}\right)$ in (3.35), we obtain:

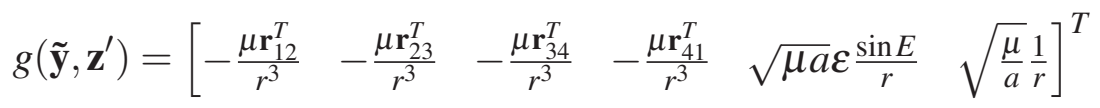

Then, the estimate state vector, $\mathbf{z}$ is:

$$
\begin{aligned}
& \mathbf{z}=\left[\begin{array}{lllll}
\mathbf{r}_{12} & \mathbf{r}_{23} & \mathbf{r}_{34} & \mathbf{r}_{41} & \dot{\mathbf{r}}_{12}
\end{array}\right. \\
& \left.\begin{array}{lllll}
\dot{\mathbf{r}}_{23} & \dot{\mathbf{r}}_{34} & \dot{\mathbf{r}}_{41} & r & E
\end{array}\right]^{T}
\end{aligned}
$$

The linear matrices $A, B$, and $G$ in (3.11) associated with state models in (3.38) and (3.39) correspond to: 


$$
\begin{gathered}
A=\left[\begin{array}{lll}
\mathbf{0}_{12 \times 12} & \mathbf{I}_{12 \times 12} & \mathbf{0}_{12 \times 2} \\
\mathbf{0}_{14 \times 12} & \mathbf{0}_{14 \times 12} & \mathbf{0}_{14 \times 2}
\end{array}\right] \\
B=\left[\begin{array}{ll}
\mathbf{0}_{14 \times 12} & \mathbf{I}_{14 \times 14}
\end{array}\right]^{T} \\
G=\left[\begin{array}{ll}
\mathbf{0}_{14 \times 12} & \mathbf{I}_{14 \times 14}
\end{array}\right]^{T}
\end{gathered}
$$

For the WLPS-only measurement, the $C$ matrix in (3.12) associated with the measurement model in (3.6) is:

$$
C=\left[\begin{array}{ll}
\mathbf{I}_{12 \times 12} & \mathbf{0}_{12 \times 14}
\end{array}\right]
$$

\subsubsection{Scenario Two - Relative Position Estimation with WLPS and Radar measurement.}

Consider a radar tracks one of the spacecraft in the two-spacecraft formation, e.g. $i^{\text {th }}$ spacecraft where $i$ represents either spacecraft 1 or spacecraft 2 . The absolute distance to the spacecraft is measured at all times. Then, the range and eccentric anomaly pseudo-measurements in (3.39) can be ommitted. Therefore, the state vector for the second scenario becomes:

$$
\mathbf{z}=\left[\begin{array}{llllllll}
\mathbf{r}_{12}^{T} & r_{i} & \lambda_{i} & \xi_{i} & \dot{\mathbf{r}}_{12}^{T} & \dot{r}_{i} & \dot{\lambda}_{i} & \dot{\xi}_{i}
\end{array}\right]^{T}
$$

where, $r_{i}$ is the absolute range, $\lambda_{i}$ is the absolute azimuth angle, $\xi_{i}$ is the absolute elevation angle, and $\dot{r}_{i}, \dot{\lambda}_{i}, \dot{\xi}_{i}$ are their time derivatives, respectively. Based on (3.44), the nonlinear function are the relative acceleration and the acceleration in terms of $\ddot{r}_{i}, \ddot{\lambda}_{i}$ and $\ddot{\xi}_{i}$. Then, the modified input function, $g\left(\tilde{\mathbf{y}}, \mathbf{z}^{\prime}\right)$ is:

$$
g\left(\tilde{\mathbf{y}}, \mathbf{z}^{\prime}\right)=\left[\frac{\mu \mathbf{r}_{1}^{T}}{r_{1}^{3}}-\frac{\mu \mathbf{r}_{2}^{T}}{r_{2}^{3}} \quad \ddot{r}_{i} \quad \ddot{\lambda}_{i} \quad \ddot{\xi}_{i}\right]^{T}
$$

where $\ddot{r}_{i}, \ddot{\lambda}_{i}$, and $\ddot{\xi}_{i}$ are the spacecraft polar accelerations, which are the second order time derivatives of the absolute range, absolute azimuth, and elevation shown in (3.3) - (3.5) respectively. Their corresponding equations are: 


$$
\begin{gathered}
\ddot{r}_{i}=r_{i} \dot{\xi}_{i}^{2}+r_{i} \dot{\lambda}_{i}^{2} \cos ^{2} \xi_{i}-\frac{\mu}{r_{i}^{2}} \\
\ddot{\lambda}_{i}=-\frac{2 \dot{r}_{i} \dot{\lambda}_{i}}{r_{i}}+2 \dot{\lambda}_{i} \dot{\xi}_{i} \tan \xi_{i} \\
\ddot{\xi}_{i}=-\frac{2 \dot{r}_{i} \dot{\xi}_{i}}{r_{i}}-2 \dot{\lambda}_{i}^{2} \cos \lambda_{i} \sin \xi_{i}
\end{gathered}
$$

where the first order time derivation of polar coordinate and cartesian coordinate are presented in Appendix A.

In this case, the $A, B, G$, and $C$ matrices for DG estimation's state and measurement models in (3.11) and (3.12), are:

$$
\begin{aligned}
& A=\left[\begin{array}{ll}
\mathbf{0}_{6 \times 6} & \mathbf{I}_{6 \times 6} \\
\mathbf{0}_{6 \times 6} & \mathbf{0}_{6 \times 6}
\end{array}\right] \\
& B=\left[\begin{array}{ll}
\mathbf{0}_{6 \times 6} & \mathbf{I}_{6 \times 6}
\end{array}\right]^{T} \\
& G=\left[\begin{array}{ll}
\mathbf{0}_{6 \times 6} & \mathbf{I}_{6 \times 6}
\end{array}\right]^{T} \\
& C=\left[\begin{array}{ll}
\mathbf{I}_{6 \times 6} & \mathbf{0}_{6 \times 6}
\end{array}\right]
\end{aligned}
$$

The process noises in the $G$ matrix in (3.51) are assumed along the acceleration axes only.

\subsection{Implementation of Extended Kalman Filter}

The standard EKF has been implemented for both scenarios. The state model, (2.3), and the measurement models, (3.6) and (3.9), correspond to the first and second scenarios respectively. In section 2.4, the EKF has been derived for the WLPS-measurements expressed in polar coordinates.

For $n$-number of spacecraft in the formation, the state estimate vector for EKF is:

$$
\hat{\mathbf{x}}=\left[\begin{array}{llllllll}
\mathbf{r}_{1}^{T} & \mathbf{r}_{2}^{T} & \ldots & \mathbf{r}_{n}^{T} & \mathbf{v}_{1}^{T} & \mathbf{v}_{2}^{T} & \ldots & \mathbf{v}_{n}^{T}
\end{array}\right]^{T}
$$


For the first scenario, the measurement model is:

$$
H_{k_{1}}=\left[\begin{array}{cccc}
-\mathbf{I}_{3 \times 3} & \mathbf{I}_{3 \times 3} & \mathbf{0}_{3 \times 6} & \mathbf{0}_{3 \times 6} \\
\mathbf{0}_{3 \times 6} & -\mathbf{I}_{3 \times 3} & \mathbf{I}_{3 \times 3} & \mathbf{0}_{3 \times 6} \\
\mathbf{0}_{3 \times 6} & \mathbf{0}_{3 \times 6} & -\mathbf{I}_{3 \times 3} & \mathbf{I}_{3 \times 3} \\
\mathbf{I}_{3 \times 3} & \mathbf{0}_{3 \times 6} & \mathbf{0}_{3 \times 6} & -\mathbf{I}_{3 \times 3}
\end{array}\right]
$$

For the second scenario, the linearized measurement model is:

$$
H_{k_{2}}=\left[\begin{array}{ccc}
-\mathbf{I}_{3 \times 3} & \mathbf{I}_{3 \times 3} & \mathbf{0}_{3 \times 6} \\
\frac{\partial h_{\text {radar }}}{\partial \mathbf{r}} & \mathbf{0}_{3 \times 3} & \mathbf{0}_{3 \times 6}
\end{array}\right]
$$

where $\frac{\partial h_{\text {radar }}}{\partial \mathbf{r}_{i}}$ is the Jacobian of the three radar measurement components introduced in (3.3) with respect to the $i^{\text {th }}$ spacecraft absolute positions and velocities [82], which is given by:

$$
\frac{\partial h_{r a d a r}}{\partial \mathbf{r}}=\left[\begin{array}{crc}
r_{i, x} / r_{i} & r_{i, y} / r_{i} & r_{i, z} / r_{i} \\
\frac{-\sin \lambda_{r i}}{r_{i} \cos \xi_{r i}} & \frac{\cos \lambda_{r i}}{r_{i} \cos \xi_{r i}} & 0 \\
\frac{-\cos \lambda_{r i} \sin \xi_{r i}}{r_{i}} & \frac{-\sin \lambda_{r i} \sin \xi_{r i}}{r_{i}} & \frac{\cos \xi_{r i}}{r_{i}}
\end{array}\right]
$$

where $\mathbf{r}_{i}=\left[\begin{array}{lll}r_{i, x} & r_{i, y} & r_{i, z}\end{array}\right]^{T}$, and $r_{i}, \lambda_{r i}$, and $\xi_{r i}$ are defined in equation (3.3).

Using the measurement noise covariance obtained in (3.7) and (3.10) together with the linearized state and measurement models, the EKF for relative position estimation is implemented.

\subsection{Simulation Results and Discussions}

Simulations are conducted to study and compare the accuracy and convergence performance between Differential Geometric Estimation and EKF. Two Differential Geometric Estimation methods discussed in Section 3.3 are implemented in the simulation, which are the pole placement and the linear filter. The accuracy performance measured is their Root Mean Square error (RMSE). Here, the Mean Square Error (MSE) is the average of the square of estimation error over $x, y$, and $z$ axes of spacecraft position. 
Thus,

$$
R M S E \equiv \frac{\left\|\hat{\mathbf{r}}_{i}-\mathbf{r}_{i}\right\|}{\sqrt{3}}
$$

where $\hat{\mathbf{r}}_{i}$ is the estimated absolute position of the $i^{t h}$ spacecraft and $\mathbf{r}_{i}$ is the true absolute position of the $i^{\text {th }}$ spacecraft.

The convergence rate is determined by the amount of time required by the estimator's RMSE to fall within a RMSE threshold, $e_{\text {thres }}$. The $e_{\text {thres }}$ is determined as the average RMSE at the estimator's RMSE steady state. In addition, the complexity of DG

Estimation and EKF are compared through the number of multiplications required by each estimator in an iteration.

In the simulation, all spacecraft are in Low Earth Orbit (LEO), with a semimajor-axis of $7000 \mathrm{~km}$, the eccentricity of 0.04 , and 0 degree of initial true anomaly. The argument of perigee (ARGPER), the Right Ascension of the Ascending Node (RAAN) and the inclination (INC) of each spacecraft are specified in each scenario in order to meet the formation configuration requirements.

The initial attitude of each spacecraft is constructed using a Euler Angle with 3-1-3 orientation at periapsis [74]. The first rotation is the spacecraft RAAN angle, followed by a second rotation (inclination angle). Then, the third rotation angle is 90 degrees for spacecraft 1,3 , and 4, and -90 degrees for spacecraft 2 . To ensure that one of the spacecraft reference frame axes points toward the center of the Earth at all times, all spacecraft are set to rotate at a constant angular velocity, which is, $\omega^{i}=\left[\begin{array}{lll}0 & 0 & \omega\end{array}\right]^{T}$. Here, $\omega^{i}$ is the angular velocity vector expressed in the $i^{t h}$ spacecraft reference frame. Then, the angular velocity of spacecraft expressed in ECI is, $\omega^{N}=D_{i}^{T} \omega^{i}$. The mean motion is $\omega=\sqrt{\mu / a^{3}}$; where $\mu$ is the earth grativational constant and $a$ is the semimajor axis.

The simulation assumptions are as follows: (i.) Process and measurement noises for all spacecraft are zero-mean Gaussian; (ii.) Initial conditions of spacecraft are well known; (iii.) The attitude of each spacecraft is well-known; (iv.) All spacecraft are equipped with a DBS and TRX; thus, each one of them can localize others; (v.) The reference frame of the DBS is aligned with the corresponding spacecraft's attitude reference frame; (vi.) The radar system measurement is available at all times for the second scenario; (vii.) no signal transmission delay; and, (viii.) The process noise variance is $10^{-7} \mathrm{~km} / \mathrm{s}^{2}$ along each axis for all scenarios. 


\subsubsection{Observability analysis}

The pole placement method presented in Section 3.3.2 requires the system to be fully observable. In this section, the system observability analysis of these two scenarios is presented. For simplicity, we consider a two-spacecraft formation case for the first scenario in this observability analysis. The estimated states for DG estimation, after the

transformation, is $\mathbf{z}=\left[\begin{array}{llll}\mathbf{r}_{12}^{T} & \mathbf{v}_{12}^{T} & r & E\end{array}\right]^{T}$. The $A$ matrix for the dynamic model in (3.11) is:

$$
A=\left[\begin{array}{ll}
I_{3 \times 3} & \mathbf{0}_{3 \times 5} \\
\mathbf{0}_{5 \times 3} & \mathbf{0}_{5 \times 5}
\end{array}\right]
$$

We assume that only relative position is measured in Cartesian coordinates. The $C$ matrix is given as:

$$
C=\left[\begin{array}{ll}
I_{3 \times 3} & \mathbf{0}_{3 \times 5}
\end{array}\right]
$$

The rank of the observability matrix using (3.27) is three, which is not full rank. Therefore, the pole placement method presented in Section 3.3.2 is not applicable.

In the second scenario, a radar measures one spacecraft's absolute position (see Eqs. (3.9) and (3.44)). Both the $A$ and $C$ matrix are given in the Eqs. (3.49) and (3.52) respectively. The rank of the observability matrix is twelve, which is full rank. Then, the pole placement method will be implemented in this scenario and compared with other estimation methods.

\subsubsection{Scenario One}

Consider only WLPS measurements are available in the four-spacecraft formation. The measurements vector, state vector, and its corresponding nonlinear model and matrices are given in Eqs.(3.6), (3.38) to (3.42) respectively. Three formation configurations that represent the short range $(\simeq 0.25 \mathrm{~km})$, medium range $(\simeq 60 \mathrm{~km})$, and long range $(\simeq 1200 \mathrm{~km})$ are considered. The spacecraft formation configurations for different formation size are shown in Table 3.1. We study the impact of formation size on both EKF and DG Estimation performance.

The measurement noise of WLPS is assumed to be 1 meter in distance (computed based on TOA measurement) and 0.001 degree in DOA. Then, the measurement noise 
Table 3.1

Spacecraft formation's configuration for short, medium and long range formation.

\begin{tabular}{|c|c|c|c|c|}
\hline \multirow{2}{*}{} & \multicolumn{4}{|c|}{ Short } \\
\cline { 2 - 5 } & S/C 1 & S/C 2 & S/C 3 & S/C 4 \\
\hline RAAN (deg) & 0 & 0 & 0 & 0 \\
\hline INC (deg) & 15 & 15 & 15 & 15 \\
\hline ARGPER (deg) & 0 & 0.001 & -0.001 & 0.002 \\
\hline & \multicolumn{5}{|c|}{ Medium } \\
\cline { 2 - 5 } & S/C 1 & S/C 2 & S/C 3 & S/C 4 \\
\hline RAAN (deg) & -0.1 & 0 & 0 & 0.1 \\
\hline INC (deg) & 14.5 & 14 & 15.5 & 15 \\
\hline ARGPER (deg) & 0.2 & 0 & -0.1 & 0.1 \\
\hline & \multicolumn{5}{|c|}{ Long } \\
\cline { 2 - 5 } & S/C 1 & S/C 2 & S/C 3 & S/C 4 \\
\hline RAAN (deg) & 0.2 & 0 & -0.1 & 0.1 \\
\hline INC (deg) & 15 & 20 & 25 & 10 \\
\hline ARGPER (deg) & 0 & 5 & -5 & 10 \\
\hline
\end{tabular}

Table 3.2

Comparing Mean RMSE in Absolute Position: DG Est. vs EKF.

\begin{tabular}{|c|c|c|}
\hline Distance & DG Est. & EKF \\
\hline Short $(\simeq 0.25 \mathrm{~km})$ & $4.447 \times 10^{3} \mathrm{~km}$ & $2.657 \times 10^{-4} \mathrm{~km}$ \\
\hline Medium $(\simeq 60 \mathrm{~km})$ & $16.59 \mathrm{~km}$ & $4.153 \times 10^{-4} \mathrm{~km}$ \\
\hline Long $(\simeq 1200 \mathrm{~km})$ & $0.901 \mathrm{~km}$ & $7.616 \times 10^{-3} \mathrm{~km}$ \\
\hline
\end{tabular}

covariance matrix is constructed using (3.7).

The process noise parameter, $\mathbf{w}$ in (2.3) and (3.11), is assumed to be $10^{-7}$ on each absolute and relative acceleration axis for both EKF and DG Estimation. The initial states standard deviations for EKF are $1 \mathrm{~km}$ and $\sqrt{0.5} \mathrm{~km} / \mathrm{sec}$ in absolute position and velocity respectively. The initial states standard deviations for DG Estimation are $1 \mathrm{~km}$ in relative position, $0.5 \mathrm{~km} / \mathrm{sec}$ in relative velocity, $2 \mathrm{~km}$ in absolute range, 0.001 degree in eccentric anomaly, and $0.001 \mathrm{~km} / \mathrm{sec}$ for the pseudo errors. 


\subsubsection{Discussion - Scenario One}

Table 3.2 shows the mean RMSE in absolute position for DG Estimation and EKF with respect to different mean relative distances. The results show that the performance of DG Estimation improves as the mean relative distance increases when the measurements consist of WLPS only. In Figure 3.1, the signal-to-noise ratio (SNR) between estimation error and relative distance decreases when the spacecraft in the formation gets closer (or 1/SNR increases), in which case the errors in the transformation from relative positions to absolute positions using (3.32) become significant.

Table 3.2 depicts that the EKF has a better accuracy compared to DG Estimation, when only WLPS measurements are used. The EKF does not require the inverse transformation (3.31). However, when the relative distance between spacecraft increases, the effect of DOA estimation error on the positioning error increases (see Figure 3.2). Accordingly, the performance of EKF drops slightly as the mean relative distance increases.

If only one DBS is available in a three-spacecraft configuration [16], the EKF would be unstable when the mean relative distance between spacecraft falls within a certain range $(\leq 10 \mathrm{~km})$. EKF stability can be improved by increasing the total number of DBSs installed in the formation, which is shown in Table 3.2. In addition, increasing the number of DBSs improves the estimation accuracy.

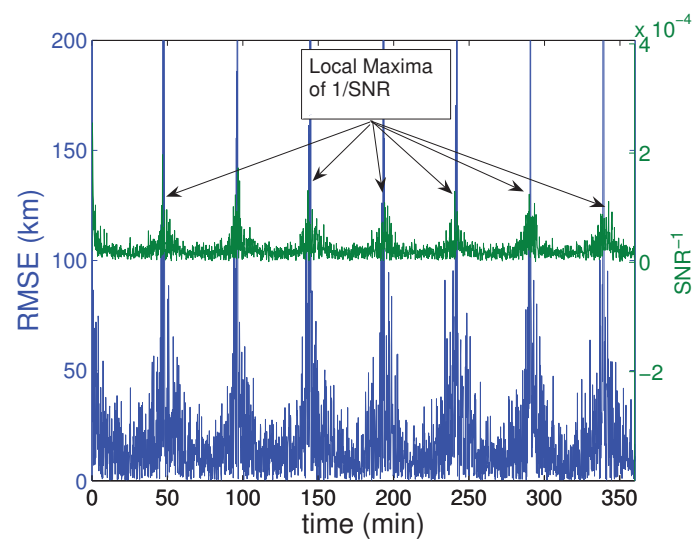

Figure 3.1: DG Est. Noise to Signal Ratio.

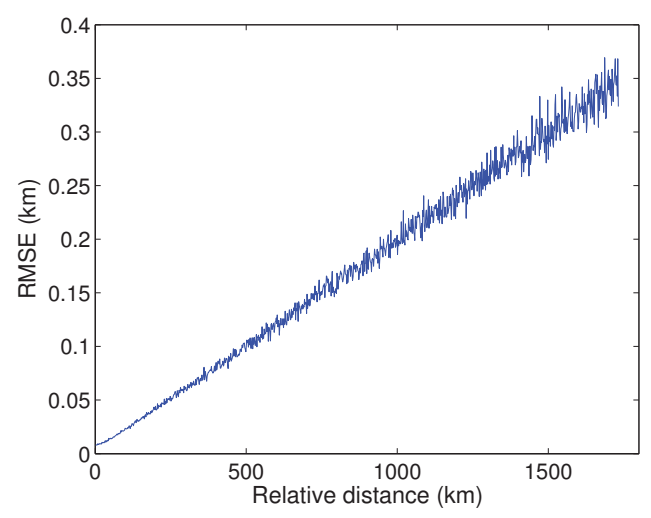

Figure 3.2: Measurement error wrt. distance. 
Table 3.3

Measurement noise's set configuration.

\begin{tabular}{|c|c|c|c|}
\hline & Minimum & Maximum & Increment \\
\hline Relative Distance, $(\mathrm{km})$ & 0.001 & 0.04 & 0.004 \\
\hline $\begin{array}{c}\text { Relative Azimuth } \\
\text { and Elevation, }(\mathrm{deg})\end{array}$ & 0.001 & 0.04 & 0.004 \\
\hline Absolute Range, $(\mathrm{km})$ & 0.1 & 4 & 0.4 \\
\hline $\begin{array}{c}\text { Absolute Azimuth } \\
\text { and Elevation, }(\mathrm{deg})\end{array}$ & 0.001 & 0.4 & 0.004 \\
\hline
\end{tabular}

\subsubsection{Scenario Two}

Consider WLPS and radar measurements are available. Here, the two-spacecraft formation with only the medium range formation configuration is considered. The radar is measuring the absolute range, azimuth, and elevation angles of spacecraft 1 at all time. The configuration of both spacecraft is similar to the configuration of S/C 1 and S/C 2 shown in Table 3.1. The measurement vector, state vector, and its corresponding nonlinear model and matrices are given in Eqs. (3.9), (3.44) to (3.52) respectively. The performance of the EKF and the DG Estimation for a given range of measurement noise is studied. The measurement noises of WLPS and radar are depicted in Table 3.3. The measurement noise covariance matrix is constructed using Eq. (3.10).

The process noise parameter, $\mathbf{w}$, is assumed to be $10^{-7}$ for all axes in EKF. For DG Estimation, the standard deviation of process noises for the relative position acceleration is $10^{-7}$, the absolute distance's acceleration is $\sqrt{10} \times 10^{-4}$, and the absolute azimuth and elevation angles' acceleration is $\sqrt{10} \times 10^{-7}$. The initial states standard deviations for EKF are $2 \mathrm{~km}$ in absolute position and $0.1 \mathrm{~km} / \mathrm{sec}$ in absolute velocity. The initial states standard deviations for DG Estimation are $0.1 \mathrm{~km}$ in relative position, $0.05 \mathrm{~km} / \mathrm{sec}$ in relative velocity, $1 \mathrm{~km}$ in absolute range, and 0.05 degrees in azimuth and elevation angles. In addition, the initial variance for the rate of change of the range is $0.1 \mathrm{~km} / \mathrm{sec}$, and the rate of change of the absolute azimuth and elevation angles is 0.001 degree.

The simulation for each set of corresponding measurement noise is repeated 10 times to compare the consistency of results produced by both filters. Then the maximum, minimum, and mean RMS of each set of measurement noise for both EKF and DG Estimation are compared. 


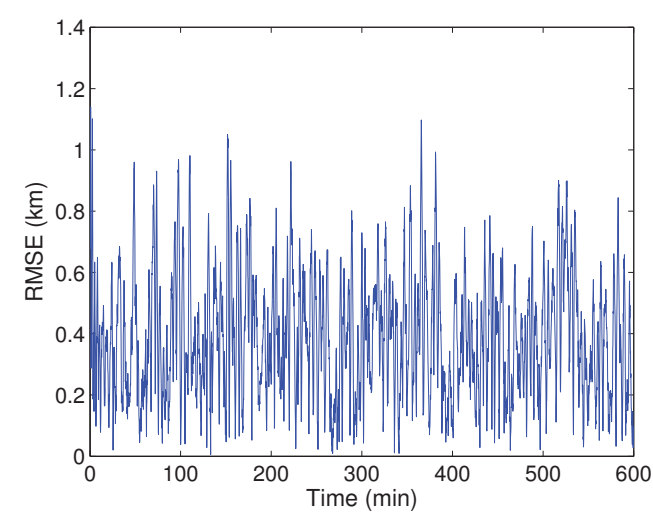

Figure 3.3: DG Est. RMSE.

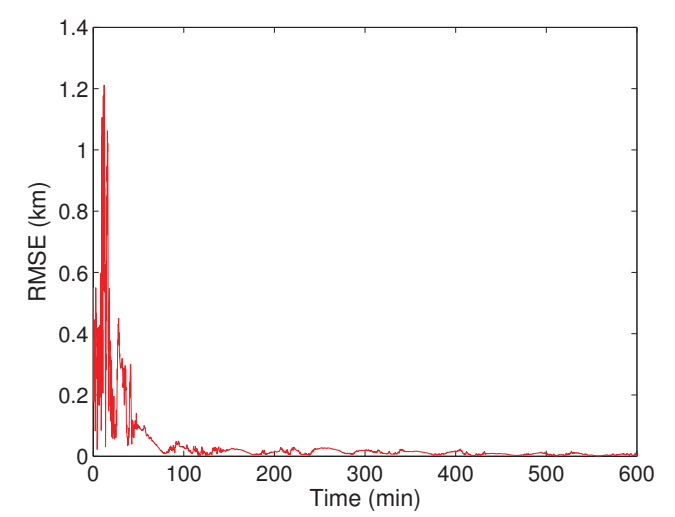

Figure 3.4: EKF RMSE.

\subsubsection{Linear Filtering Gain}

First, the DG Estimation is compared with the EKF performance using the gain computation presented in Section 3.3.1. Figures 3.3 and 3.4 show the RMSE of DG Estimation and EKF for measurement noise standard deviation of $0.001 \mathrm{~km}$ in relative range, 0.001 degree in relative azimuth and elevation angle, $0.01 \mathrm{~km}$ in absolute range, and 0.01 degree in absolute azimuth and elevation angle. Figure 3.3 shows that the DG Estimation converges faster than EKF, because the DG Estimation avoids the linearization steps in the estimation.

However, Figure 3.4 shows that EKF is more accurate than DG Estimation. The nonlinear dynamic model in DG estimation is expressed in terms of measurements and pseudo-measurements (see (3.38) and (3.45)). While the linearization step is eliminated in the DG estimator, the propagation step is dependent on the measurements noises. On the other hand, the nonlinear dynamic model of EKF is expressed in terms of the estimated state vector with additional linearization steps. The EKF is able to achieve a better accuracy if the estimation converges.

Figure 3.5 compares the minimum and maximum RMSE of DG Estimation and EKF for a given set of measurement noise standard deviation. The DG gain is computed using the method shown in Section 3.3.1. With additional radar measurement, the DG Estimation accuracy is improved. In addition, the minimum and maximum RMSE of DG Estimation are very close compared to EKF because the DG estimation does not require linearization steps. Therefore, a stable estimation can be always achieved. 


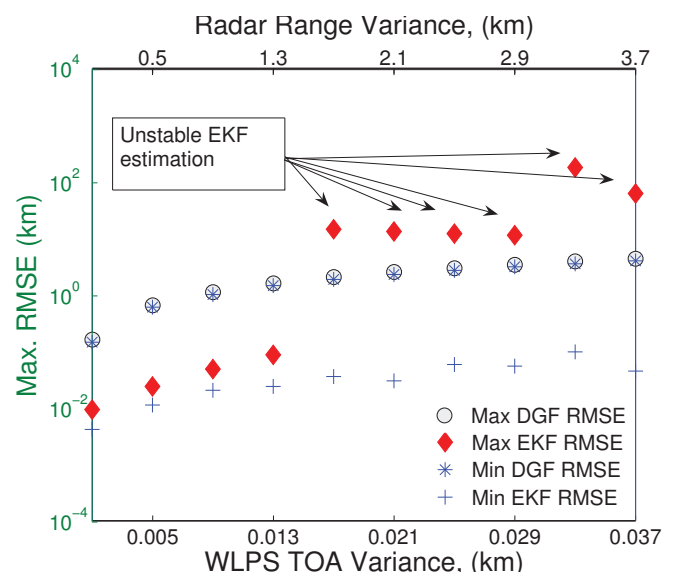

Figure 3.5: Minimum and Maximum Mean RMSE Comparison between two filters with respect to measurement noise increment.

Figure 3.5 also shows that the EKF has better accuracy performance than the DG estimation. However, the stability of the EKF is not always guaranteed. In Figure 3.5, we observe large RMSEs in the EKF estimation at higher measurement noise levels. The large RMSE is caused by the unstable estimation of EKF, which is due to the linearization of the nonlinear radar measurement and state models in the EKF algorithm. On the other hand, the DG Estimation does not require any linearization step.

\subsubsection{Pole Placement Method}

Figures 3.6 and 3.7 compare the RMSE performance between the DG Estimation and the EKF. The EKF's configuration is the same as in the previous case. On the other hand, the

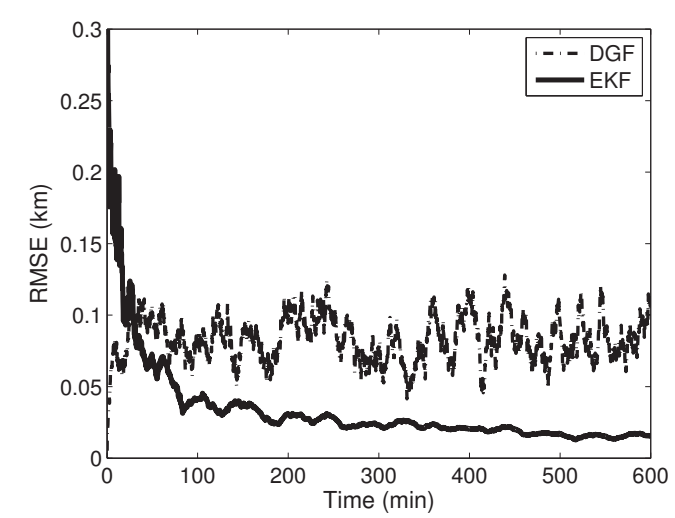

Figure 3.6: RMSE Comparison (Stable EKF).

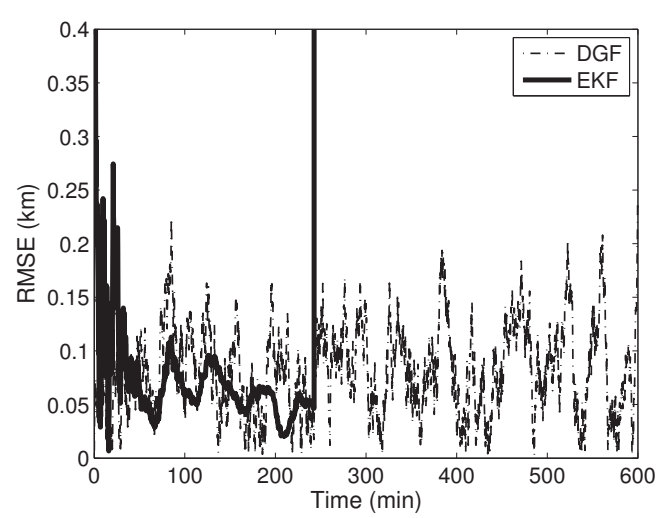

Figure 3.7: RMSE Comparison (Unstable EKf). 
pole placement method is as follows. The poles for the relative position are $\left[\begin{array}{lll}-0.001 & -0.001 & -0.001\end{array}\right]$, for the relative velocity vector are $\left[\begin{array}{lll}-0.0004 & -0.0004 & -0.0004\end{array}\right]$, and for the absolute position and velocity in polar coordinates are $\left[\begin{array}{lll}-0.1 & -0.02 & -0.02\end{array}\right]$ and $\left[\begin{array}{lll}-0.05 & -0.01 & -0.01\end{array}\right]$ respectively. The DG estimation gain is computed using the Ackermann's formula [22].

Figure 3.6 shows that when the EKF estimation is stable, the EKF has better accuracy performance than the DG Estimation. However, the linear system characteristic of the DG Estimation allows it to converge faster than the EKF. Figure 3.7 shows that the EKF may suffer instability due to the linearization steps in the algorithm. In Figure 3.7, the EKF is able to converge to lower RMSE than the DG Estimation, but the RMSE diverges after a certain time period. However, the DG Estimation does not suffer any instability. Therefore, the pole placement method in DG Estimation does guarantee the fast convergence and stability of estimation algorithm.

\subsubsection{Complexity Analysis}

In [85], a formalism of $\mathscr{O}$ function for complexity computational of matrices operation has been introduced, which are:

$$
\begin{gathered}
\operatorname{SUM}(N \times M, N \times M)=\mathscr{O}(N \times M) \\
\operatorname{SUB}(N \times M, N \times M)=\mathscr{O}(N \times M) \\
\operatorname{MUL}(N \times M, M \times P)=\mathscr{O}(N \times M \times P) \\
\operatorname{INV}(N \times N)=\mathscr{O}\left(N^{3}\right)
\end{gathered}
$$

where, $M, N$, and $P$ are the dimensions of matrices that perform these operations. Here, $P$ is the number of states in DG Estimation, $N$ is the number of states in EKF, $M$ is the number of measurements, and $Q_{p}$ and $Q_{n}$ are the number of process noise in DG Estimation and EKF, respectively.

We assume the computational complexity of the Jacobian matrices is in the order of $\mathscr{O}(1)$. In addition, the propagation of the state models are considered to have the complexity of $\mathscr{O}(1)$ in both filters. The EKF requires an order of $\mathscr{O}(M \times N \times N)$ for measurement model computation, while the DG estimation only requires an order of $\mathscr{O}(1)$ computation 
complexity. Therefore, the EKF requires higher computational complexity to compute the measurement.

The EKF requires the order of $\mathscr{O}\left(M^{3}\right)$ and $\mathscr{O}\left(M \times N^{2}\right)$ computation complexity for gain matrix. There are two different computation complexities in DG estimation for gain matrix computation. For the pole placement method, there is no computational complexity because the gain matrix is pre-determined. For the linear filtering method, the gain computational complexity is in the order of $\mathscr{O}\left(M^{3}\right)$ and $\mathscr{O}\left(M \times P^{2}\right)$. Both EKF and DG estimation may have same computation complexity if all states are observed. However, the DG estimation has higher computational complexity if not all states are observed $\left(P^{2} \gg N^{2}\right)$.

The covariance update and propagation's computational complexity for the EKF are in the order of $\mathscr{O}\left(N^{3}\right), \mathscr{O}\left(N^{2} \times Q_{n}\right)$ and $\mathscr{O}\left(N^{3}\right)$. There is no computational complexity for covaraince update and propagation for DG estimation if the pole placement method is used. However, for the linear filtering method, the computational complexities are in the order of $\mathscr{O}\left(P^{3}\right), \mathscr{O}\left(P^{2} \times Q_{p}\right)$ and $\mathscr{O}\left(P^{3}\right)$.

Therefore, for the fully observable case, the DG estimation has lower computational complexity. In addition, among the two DG estimation approaches implemented in this chapter, the pole placement method has lower computational complexity compared to the linear filtering method. But the EKF has lower computation complexity if not all states are fully observed. 


\section{Chapter 4}

\section{Constraint Estimation of Spacecraft}

\section{Positions}




\subsection{Introduction}

The estimation of relative and absolute positions of spacecraft formations is a fundamental task in many space missions. ${ }^{*}$ Relative position estimation plays an important role in Spacecraft Formation Flying (SFF) missions, a subject that has received much attention by researchers during the last few decades. Some SFF missions require that multiple spacecraft, in different orbits, communicate without interruption, e.g., satellites in the CITRIS-COSMIC system are required to communicate with each other to monitor the ionospheric irregularities [1]. Relative positions between satellites, such as Cluster and Cluster-II satellites that are launched by the European Space Agency, are estimated and controlled to support many collaborative tasks where satellites are required to maintain a specific formation in a continuous manner within the mission period [2].

The Extended Kalman Filter (EKF) has been widely implemented to estimate absolute and relative positions of spacecraft in formation flying $[7,16]$. The initial condition of the estimation of the SFF affects the stability of EKF. Also, the Unscented Kalman Filter (UKF) has been implemented for relative attitude and position estimation in SFF [17, 18]. The UKF has better robustness to initial condition error compared to the EKF [19]. However, its computational time is higher than a standard EKF [20]. If compared to the standard EKF, the UKF has a faster convergence rate, but the process time required for each update is longer than the EKF [21]. The Batch filter [22], an offline filtering method, has been implemented in Ref. [5] for absolute position estimation using the relative position measurement. Both studies in references [16] and [5] show that the configuration of SFF affects the stability and the accuracy of estimation.

The Constrained Kalman Filter (CKF) was introduced for estimation when state variables are required to satisfy constraints $[23,24]$. Both equality and inequality constraints can be handled by the CKF. Several CKF algorithms have been proposed in the literature, e.g., the perfect measurement approach [25, 26], and the projection approach [27]. Some of the CKF methods may require special derivations for different problems, such as the reduction method [28] and the Norm-Constrained method in Ref. [29].

In the perfect measurement approach, the constraint is handled as a perfectly known measurement. The constraint is grouped with other sensor measurements to construct a

*The material contained in this chapter has been accepted for publication in the Journal of Guidance, Navigation and Control. 
modified measurement vector, which affects the calculation of the Kalman gain [23]. Adding a perfect measurement (constraint) results in a singular noise covariance matrix. This singularity in the noise covariance may lead to a divergence in the estimation error. This fact was pointed out in references [30] and [31], despite that, the original work of Kalman [14] presents an example in which a perfect measurement was used [23]. To overcome this singularity problem, Ref. [32] presents an extended maximum likelihood method for computing the constraint gain.

In the projection approach, the constraint estimation is implemented by projecting the unconstrained state estimate onto the constraint surface. In general, the constraint update is applied after the standard Kalman filter update [23]. Ref. [23] has presented three different constraint update methods that can be used for the projection approach. However, different approximated solutions may be obtained from each method because the constraint gain is not correlated with the sensor measurement [23]. The projection approach has also been extended for inequality constraint [33] and nonlinear constraint [27] problems.

The Norm-Constrained method presented in Ref. [29] guarantees non-singular estimation and correlates both the measurement and the constraint. However, the derivation of the Kalman gain is specific to the attitude estimation problem, and becomes rather complex when applied for the problem presented in this chapter. The reduction method requires that one or more state(s) be expressed as a linear function(s) of the other states. However, this method may not be applicable for the cases where the constraint states are independent from each other. Ref. [34] presents a Smoothly CKF (SCKF) where the constraint update ia carried out iteratively until an end criteria is met. Ref. [34] shows that the SCKF outperforms the Iterated EKF in terms of consistency.

The implementation of constraint estimation for attitude kinematics of spacecraft has been widely studied by researchers. Ref. [86] presents an implementation of the SCKF to improve angular motion estimation by using an orthogonal acceleration constraint. Different approaches have been developed for implementing CKF to improve spacecraft attitude estimation [29, 87, 88].

Ref. [89] and [90] present the implementation of constraint estimation to improve the GPS/INS navigation system of a ground vehicle. In Ref. [89], the constraint estimation is applied if the road geometry from the navigation system's digital map is known. On the other hand, Ref. [90] presents the implementation of a dynamic-aided method into the 
vehicle's navigation system to improve the GPS/INS navigation performance during a long GPS outage. Multiple constraints are addressed under each dynamic motion of the vehicle. The corresponding constraint is applied if the vehicle meets a certain dynamic motion criteria. The paper shows that the dynamic-aided method improves navigation performance during a long GPS outage [90].

Reference [91] presents an orbit determination method for spacecraft formation, known as the Link, Autonomous, Interplanetary Satellite Orbit Navigation (LiAISON). The LiAISON method uses a three-body dynamic model. Solar radiation pressure, non-symmetric gravity fields, and third-body gravity create asymmetry in the acceleration model and lead to unique or locally unique orbits. The uniqueness of orbits helps the determination of absolute orbits using only relative measurements [91].

In this chapter, the absolute positions of the spacecraft in a formation are estimated using relative position measurements. A two-body dynamic model is used. In a two-body dynamic motion, the spacecraft range from the Earth center has two extrema: perigee and apogee points. For any orbital motion, the absolute position of spacecraft with respect to the focal point (Earth center) changes continuously. The absolute distance attains its maximum and minimum values at the apogee and perigee positions, respectively [75]. At these points, the rate of change of the absolute distance is zero. Accordingly, a constraint estimation can be incorporated to improve the absolute position estimation accuracy. A projection approach $\mathrm{CKF}$ is implemented. Because the proposed constraints are applied at only two points in the orbit, the estimation may encounter instability. A tuning algorithm is suggested and tested on several cases.

Two scenarios are presented in this chapter. The first scenario assumes a single spacecraft and radar measurements. Few studies in the literature prove the feasibility of estimating spacecraft formation absolute positions using only relative positions measurements $[5,16,92]$. The implementation of these algorithms, however, may suffer poor estimation accuracy or instabilities in some formation flying configurations. The second scenario investigates the impact of using CKF for estimating SFF absolute positions using relative positions measurements. Relative measurements are assumed to be available using a new Wireless Local Positioning System (WLPS) [58, 81]. The WLPS enables relative position measurement between spacecraft in formation via relative range, azimuth, and elevation angle measurements $[16,58,81,93]$. We assume only WLPS is available in the SFF system. 
Three case studies are investigated in this chapter. In the first case study, all spacecraft in the formation are in the same circular orbit. In the second case study, we assume the time when the spacecraft arrive at either perigee or apogee position is known at all times.

Lastly, we assume that the time for the spacecraft to arrive at perigee or apogee position is unknown. An algorithm to determine the time required by the spacecraft to arrive at perigee and apogee position is presented in this chapter.

Section 4.2 presents the Constrained Kalman Filter. The derivations of apogee and perigee constraint for all case studies are presented in Section 4.3. Section 4.4 discusses the simulation results.

\subsection{Constrained Kalman Filter}

In this chapter, the projection approach of CKF is implemented. The nonlinear system and measurement models take the form:

$$
\begin{aligned}
\dot{\mathbf{x}} & =\mathbf{f}(\mathbf{x})+\mathbf{w} \\
\mathbf{y} & =\mathbf{h}(\mathbf{x})+v
\end{aligned}
$$

where $\mathbf{h}(\mathbf{x})$ is the nonlinear measurement model defined in Section 2.3.2.

The EKF update equations take the form:

$$
\begin{gathered}
\hat{\mathbf{x}}^{+}=\hat{\mathbf{x}}^{-}+K(\tilde{\mathbf{y}}-\hat{\mathbf{y}}) \\
P^{+}=(I-K H) P^{-}
\end{gathered}
$$

where $K$ is the Kalman filter gain,

$$
K=P^{-} H^{T}\left(H P^{-} H^{T}+R\right)^{-1}
$$

where $P^{-}$is the pre-update states covariance.

It is possible to show that the post-update state covariance, $P^{+}$, is always symmetric if $P^{-}$ is symmetric by substituting Eq. (4.5) into (4.4). If the estimated state at a particular time step, $\hat{\mathbf{x}}_{k}$, is subject to a constraint function, $\mathbf{b}=d\left(\hat{\mathbf{x}}_{k}\right)$, then the post-update state estimate 


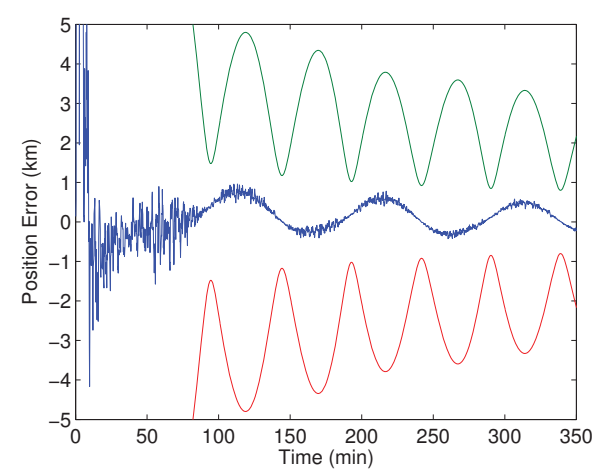

(a) EKF.

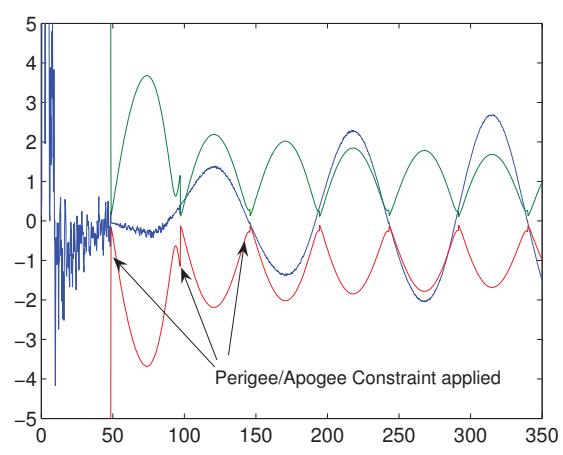

(b) CKF.

Figure 4.1: Estimation error and three sigma boundary plots for EKF and CKF

$\hat{\mathbf{x}}_{k}^{+}$and the state covariance $P_{k}^{+}$, in Eqs. (4.3) and (4.4), will be updated as follows:

$$
\begin{gathered}
\tilde{\mathbf{x}}_{k}^{+}=\hat{\mathbf{x}}_{k}^{+}+\lambda\left(\mathbf{b}-d\left(\hat{\mathbf{x}}_{k}^{+}\right)\right) \\
\tilde{P}_{k}^{+}=(I-\lambda D) P_{k}^{+}
\end{gathered}
$$

The overhead symbol tilde represents post constraint update, and $D$ is the first order linearization matrix of the given constraint function, $\left.D \equiv \frac{\partial d\left(\hat{\mathbf{x}}^{+}\right)}{\partial \mathbf{x}}\right|_{\mathbf{X}=\hat{\mathbf{x}}^{+}}$.

The constraint gain, $\lambda$, is derived in a similar way as the Kalman gain, $K$, which is given as:

$$
\lambda=P^{+} D^{T}\left(D P^{+} D^{T}+R_{d}\right)^{-1}
$$

It is noted that the constraint update is similar to the update of a perfect measurement. The $R_{d}$ in Eq. (4.8) serves as a control matrix. If $D P^{+} D^{T}$ is singular, then $R_{d}$ is assigned to a small value to avoid the singularity [94]. If $D P^{+} D^{T}$ is non-singular, then we select $R_{d}=\mathbf{0}$.

The implementation of the CKF (Eqs. (4.6) and (4.7)) in this problem results in instability in the estimation. The reason is that the constraints are applied at only two points in the orbit (perigee and apogee). Applying the constraint at the perigee and apogee positions provides additional information to the estimator and causes a jump (discontinuity) in the covariance. The discontinuity causes the covariance to converge abruptly and faster than the estimated state. Figures 4.1(a) and 4.1(b) compares the error of the estimated state for both the EKF and the CKF, with respect to the three sigma boundaries. Figure 4.1(b) 


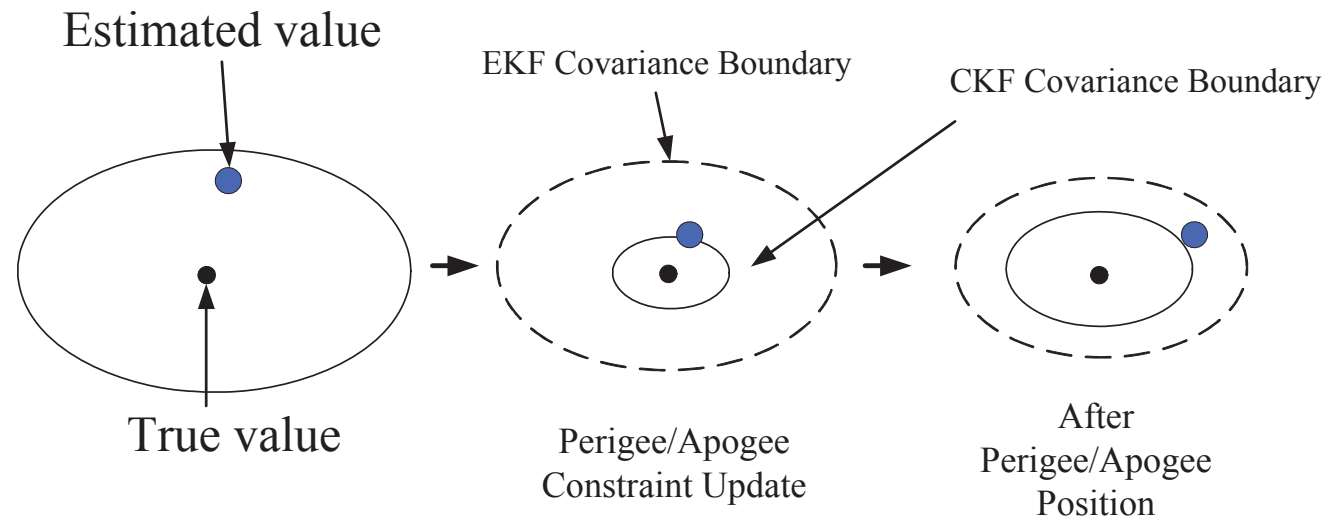

Figure 4.2: Change of covariance boundary in Kalman Filter process.

shows that the covariance boundary of the CKF significantly reduces at each perigee and apogee position. As a result, the error in the estimated state becomes higher than that predicted by the estimated covariance (state estimate error curve is outside the three sigma boundary). Thus, the system becomes unstable. Figure 4.2 shows an illustration for how the covariances of both the EKF and CKF vary when the constraint is applied. A similar covariance behavior was observed in Ref. [95].

If the EKF is run through a high number of observations and then the constraints are applied at perigee and apogee points, the results show that estimation accuracy gets worse due to constraint implementation, as shown in Figure 4.3.

To avoid the abrupt covariance convergence problem, two scalar weights, $\alpha$ and $\beta$, are

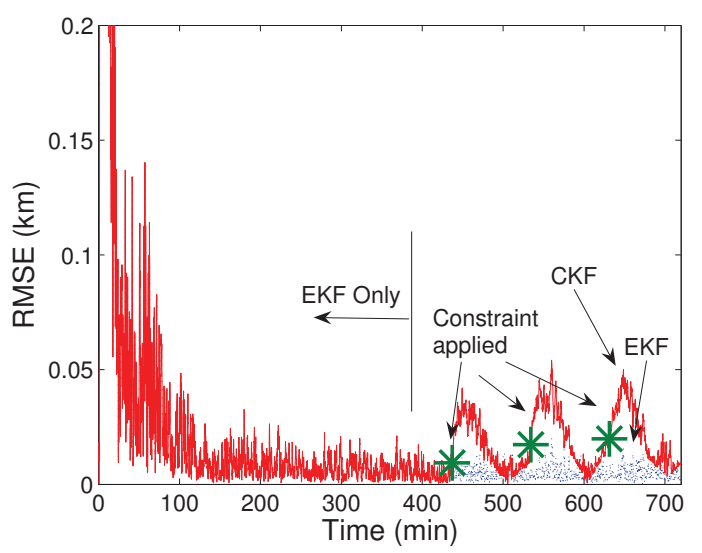

Figure 4.3: Applying perigee/apogee constraints after implementing EKF for high number of observations. 
introduced into Eqs. (4.6) and (4.7):

$$
\begin{gathered}
\tilde{\mathbf{x}}^{+}=\hat{\mathbf{x}}^{+}+\alpha \lambda\left(\mathbf{d}-D \hat{\mathbf{x}}^{+}\right) \\
\tilde{P}^{+}=(I-\beta \lambda D) P^{+}
\end{gathered}
$$

where $0<\alpha<1$ and $0<\beta<1$.

The $\alpha$ and $\beta$ parameters affect a smooth application of the constraint, through applying it partially every time a perigee/apogee is encountered. The $\beta$ parameter controls the speed of convergence of the covariance. In general, increasing the value of $\beta$ causes the covariance to converge faster due to the constraint, and decreasing the value of $\beta$ means that our confidence in the estimated state does not change much due to the constraint application. The $\alpha$ parameter controls the amplitude of update in the state, due to the application of the constraint.

In this chapter, a numerical analysis is carried out to determine the optimal values for the parameters $\alpha$ and $\beta$ for different formation altitudes and orbits. The estimation process is carried out as follows. The state estimate and its covariance are updated using the measurements (Eqs. (4.3) and (4.4)). At the time of perigee or apogee, an additional update is carried out using Eqs. (4.9) and (4.10). The estimated state vector is propagated using the dynamic model in Eq. (2.3), and the covariance is propagated using the following equation:

$$
\dot{P}(t)=P(t) F^{T}(\hat{\mathbf{x}}(t), t)+F(\hat{\mathbf{x}}(t), t) P(t)+G(t) Q(t) G(t)
$$

where $F(\hat{\mathbf{x}}(t), t)$ is the Jacobian matrix of Eq. (2.3), and $G$ corresponds to:

$$
G=\left[\begin{array}{cccc}
A & \mathbf{0}_{6 \times 6} & \cdots & \mathbf{0}_{6 \times 6} \\
\mathbf{0}_{6 \times 6} & A & \cdots & \mathbf{0}_{6 \times 6} \\
\vdots & \ddots & \ddots & \vdots \\
\mathbf{0}_{6 \times 6} & \cdots & \cdots & A
\end{array}\right]
$$

where,

$$
A=\left[\begin{array}{ll}
\mathbf{0}_{3 \times 3} & \mathbf{0}_{3 \times 3} \\
\mathbf{0}_{3 \times 3} & \mathbf{I}_{3 \times 3}
\end{array}\right]
$$


The $F(\hat{\mathbf{x}}(t), t)$ matrix, for $n$ spacecraft formation, is given as:

$$
F(\hat{\mathbf{x}}(t), t)=\left[\begin{array}{cccc}
\frac{\partial \mathbf{f}_{1}}{\partial \mathbf{x}_{1}} & \mathbf{0}_{6 \times 6} & \cdots & \mathbf{0}_{6 \times 6} \\
\mathbf{0}_{6 \times 6} & \frac{\partial \mathbf{f}_{2}}{\partial \mathbf{x}_{2}} & \cdots & \mathbf{0}_{6 \times 6} \\
\vdots & \ddots & \ddots & \vdots \\
\mathbf{0}_{6 \times 6} & \cdots & \cdots & \frac{\partial \mathbf{f}_{n}}{\partial \mathbf{x}_{n}}
\end{array}\right]
$$

where,

$$
\frac{\partial \mathbf{f}_{i}}{\partial \mathbf{x}_{i}}=\frac{\mu}{s^{5}}\left[\begin{array}{cccc}
\mathbf{0}_{3 \times 1} & \mathbf{0}_{3 \times 1} & \mathbf{0}_{3 \times 1} & I_{3 \times 3} \\
2 r_{i, x}^{2}-r_{i, y}^{2}-r_{i, z}^{2} & 3 r_{i, x} r_{i, y} & 3 r_{i, x} r_{i, z} & \mathbf{0}_{1 \times 3} \\
3 r_{i, x} r_{i, y} & 2 r_{i, y}^{2}-r_{i, x}^{2}-r_{i, z}^{2} & 3 r_{i, y} r_{i, z} & \mathbf{0}_{1 \times 3} \\
3 r_{i, x} r_{i, z} & 3 r_{i, y} r_{i, z} & 2 r_{i, z}^{2}-r_{i, x}^{2}-r_{i, y}^{2} & \mathbf{0}_{1 \times 3}
\end{array}\right]
$$

where $s=\sqrt{r_{i, x}^{2}+r_{i, y}^{2}+r_{i, z}^{2}}$, and $i=1, \ldots, n$.

\subsection{Spacecraft Position and Velocity at Perigee and Apogee}

At the perigee and apogee positions, the rate of change of the absolute distance between the spacecraft and the Earth center vanishes. We define the absolute velocity vectors as $\mathbf{v}=\left[\begin{array}{lll}v_{x} & v_{y} & v_{z}\end{array}\right]^{T}$, and the absolute distance as $r=\sqrt{r_{x}^{2}+r_{y}^{2}+r_{z}^{2}}$. The time derivative of the absolute distance is [76]:

$$
\frac{\partial r}{\partial t}=\frac{\mathbf{r}^{T} \mathbf{v}}{r}
$$

The constraints at the apogee and perigee positions are defined as:

$$
\frac{\partial r_{i, a / p}}{\partial t} \equiv \frac{\mathbf{r}_{i, a / p}^{T} \mathbf{v}_{i, a / p}}{r_{i, a / p}}=0
$$

where the subscript $i$ represents the $i^{t h}$ spacecraft, while subscript $a / p$ represents the position and velocity vector at either apogee or perigee 
Then, the constraint function for the $i^{t h}$ spacecraft, $d_{i}\left(\mathbf{x}_{i}\right)$ in (4.6), can be written as:

$$
d_{i}\left(\mathbf{x}_{i}\right)=\frac{\mathbf{r}_{i, a / p}^{T} \mathbf{v}_{i, a / p}}{\left\|\mathbf{r}_{i, a / p}\right\|}
$$

The constraint presented in Eqs. (4.17) and (4.18) can be applied at all points of a circular orbit. For elliptic orbit, it applies only at perigee and apogee. Hence, the perigee and apogee times need to be known in order to apply the constraint. Suppose that $\Delta t_{p}$ is the time of travel from the current position to the perigee. Similarly, $\Delta t_{a}$ is the time of travel from the current position to the apogee. Three cases will be studied:

1. Circular Orbit: the true orbit is assumed to be circular;

2. Elliptic orbit with known $\Delta t_{p}$ and $\Delta t_{a}$ : in this case study, it is assumed that both $\Delta t_{p}$ and $\Delta t_{a}$ are known; and

3. Elliptic orbit with unknown $\Delta t_{p}$ and $\Delta t_{a}$ : this is a more practical case where $\Delta t_{p}$ and $\Delta t_{a}$ are unknown.

\subsubsection{Circular Orbit}

For a circular orbit, the spacecraft orbits around the Earth at constant absolute distance at all times. Therefore, the constraint in Eq. (4.17) can be applied at all times. The CKF estimation algorithm is applied as follows. The estimated states and states covariance are updated using Eqs. (4.3) and (4.4). Then, both the post update estimated states and covariance are updated with respect to the constraint using Eqs. (4.6) and (4.7). After that, they are propagated to the next time step to predict the spacecraft position and the covariance using Eqs. (2.3) and (4.11).

\subsubsection{Elliptic orbit with well-known $\Delta t_{p}$ and $\Delta t_{a}$}

For any elliptic orbit, the absolute distance between spacecraft and the Earth center changes at all times. Let $\delta t$ be the time interval between two successive measurements $\left(\tilde{\mathbf{y}}_{k}\right.$ and $\left.\tilde{\mathbf{y}}_{k+1}\right)$. At a particular time step, $t_{k}$, both the estimated states and states covariance are updated using Eqs. (4.3) and (4.4). Then, the known $\Delta t_{p}$ (or $\Delta t_{a}$ ) is compared to $\delta t$. If 
$\Delta t_{p}<\delta t$ (or $\Delta t_{a}<\delta t$ ) is true, the updated estimated states and states covariance are propagated to the respective perigee (or apogee) position, followed by the constraint update using Eqs. (4.6) and (4.7). After that, the updated states and states covariance are propagated to the next time step using Eqs. (2.3) and (4.11).

\subsubsection{Elliptic orbit with unknown $\Delta t_{p}$ and $\Delta t_{a}$}

When the time to perigee (apogee), $\Delta t_{p}\left(\Delta t_{a}\right)$, is not known, it can be computed from the estimated states as follows [76]:

$$
\begin{gathered}
\Delta t_{p}=-\sqrt{\frac{\hat{a}^{3}}{\mu}}(\hat{E}-\hat{e} \sin \hat{E}) \\
\Delta t_{a}=\sqrt{\frac{\hat{a}^{3}}{\mu}}[\pi-(\hat{E}-\hat{e} \sin \hat{E})]
\end{gathered}
$$

The algorithm to compute the semimajor axis, eccentric anomaly, and eccentricity, from spacecraft position and velocity vectors can be found in several references $[75,76]$. The predicted spacecraft's position and velocity vectors at apogee (or perigee) position are carried out using the $f$ and $g$ functions representation of Kepler's motion [76]:

$$
\begin{aligned}
\mathbf{r}_{i, a / p} & =f \mathbf{r}_{i, k}+g \mathbf{v}_{i, k} \\
\mathbf{v}_{i, a / p} & =\dot{f} \mathbf{r}_{i, k}+\dot{g} \mathbf{v}_{i, k}
\end{aligned}
$$

where subscript $i$ represents $i^{t h}$ spacecraft, and subscript $k$ is the time counter.

As seen in Eqs. (4.21) and (4.22), the $f$ and $g$ functions can be used to compute a spacecraft's position and velocity at a future time. This method of computing position and velocity does not require integration, yet its accuracy is comparable to the two-body dynamic model. There are several methods to determine the $f$ and $g$ functions. Here, the Universal Variable method is implemented. The details on how to determine the Universal Variable, $\chi$, and the $f$ and $g$ functions can be found in Ref. [76].

At each measurement update, the $\Delta t_{p}\left(\Delta t_{a}\right)$ is computed. It is then compared to the time to the next measurement $(\delta t)$. If $\Delta t_{p}<\delta t\left(\Delta t_{a}<\delta t\right.$ ), then the corresponding perigee (apogee) position and velocity of the spacecraft are computed using Eqs. (4.21) and 
(4.22). At the current measurement time $k$, the state estimate and the covariance will be updated twice. The first update is that of the measurements, using Eqs. (4.3) and (4.4). The second update is that of the constraints, using Eqs. (4.6) and (4.7).

However, the expressions of $a, E$ and $e$ in Eqs. (4.19) and (4.20) are nonlinear expressions in terms of spacecraft position and velocity vectors. Also, all the $f, g, \dot{f}$, and $\dot{g}$ are nonlinear functions. Thus, a linearization is required for Eqs. (4.21) and (4.22), which is given as:

$$
\begin{aligned}
\frac{\partial d(\mathbf{x})}{\partial \mathbf{r}_{i, k}}=2 \frac{f \dot{f}}{D U^{2}} & \frac{\mathbf{r}_{i, k}^{T}}{\left\|\mathbf{r}_{i, a / p}\right\|}+(f \dot{g}+g \dot{f}) \frac{T U}{D U^{2}} \frac{\mathbf{v}_{i, k}^{T}}{\left\|\mathbf{r}_{i, a / p}\right\|} \\
& -\frac{\mathbf{r}_{i, a / p}^{2} \mathbf{v}_{i, a / p}}{\left\|\mathbf{r}_{i, a / p}\right\|^{3}}\left(\frac{f^{2}}{D U^{2}} \mathbf{r}_{i, k}^{T}+f g \frac{T U}{D U^{2}} \mathbf{v}_{i, k}^{T}\right)+\frac{d g(\Delta t, \mathbf{r}, \mathbf{v})}{d \mathbf{r}} \\
\frac{\partial d(\mathbf{x})}{\partial \mathbf{v}_{i, k}}=2 g \dot{g} \frac{T U^{2}}{D U^{2}} & \frac{\mathbf{v}_{i, k}^{T}}{\left\|\mathbf{r}_{i, a / p}\right\|}+(f \dot{g}+g \dot{f}) \frac{T U}{D U^{2}} \frac{\mathbf{r}_{i, k}^{T}}{\left\|\mathbf{r}_{i, a / p}\right\|} \\
& -\frac{\mathbf{r}_{i, a / p}^{2} \mathbf{v}_{i, a / p}}{\left\|\mathbf{r}_{i, a / p}\right\|^{3}}\left(g^{2} \frac{T U^{2}}{D U^{2}} \mathbf{v}_{i, k}^{T}+f g \frac{T U}{D U^{2}} \mathbf{r}_{i, k}^{T}\right)+\frac{d g(\Delta t, \mathbf{r}, \mathbf{v})}{d \mathbf{v}}
\end{aligned}
$$

where the derivation for both $\frac{d g(\Delta t, \mathbf{r}, \mathbf{v})}{d \mathbf{r}}$ and $\frac{d g(\Delta t, \mathbf{r}, \mathbf{v})}{d \mathbf{v}}$ are shown in Eqs. (9.19) and (9.20) (in Appendix).

\subsection{Simulation and Discussion}

Simulations are conducted to estimate the absolute positions and velocities of the spacecraft. The Root Mean Square Error (RMSE) of estimation at any time $k$ is given as:

$$
\zeta_{k}=\frac{\left\|\hat{\mathbf{r}}_{i, k}-\mathbf{r}_{k}\right\|}{\sqrt{3}}
$$

The average RMSE over time is $\bar{\zeta}$. The average RMSE is determined by computing the time average of the RMSE, after the RMSE has converged and is below a certain threshold. The threshold may vary from one problem to another.

The performance of the CKF is evaluated by computing the ratio between the average $\operatorname{RMSE}$ of the $\operatorname{CKF}\left(\bar{\zeta}_{C K F}\right)$ and the average RMSE of the EKF $\left(\bar{\zeta}_{E K F}\right)$, in the absolute 
Table 4.1

Spacecraft configuration.

\begin{tabular}{|c|ccc|}
\hline \multirow{2}{*}{ S/C \# } & \multicolumn{3}{|c|}{ Orbital Elements } \\
\cline { 2 - 4 } & RAAN, (deg.) & Inclination, (deg.) & Arg. of Perigee, (deg.) \\
\hline 1 & 0.1 & 0.4 & 0 \\
2 & 0.2 & -0.4 & 0 \\
3 & -0.2 & -0.4 & 0 \\
4 & -0.1 & -0.4 & 0 \\
\hline
\end{tabular}

position estimation. The performance ratio percentage is defined as:

$$
\text { PERF. }=\frac{\bar{\zeta}_{E K F}}{\bar{\zeta}_{C K F}} \times 100 \%
$$

If $P E R F$. is larger than $100 \%$, it means the CKF has a lower RMSE than EKF.

The simulation assumptions are as follows: (i) No signal transmission and processing time delays in measurements; (ii) Process and measurement noises for all spacecraft are zero-mean Gaussian; (iii) Initial conditions of spacecraft are well known; (iv) The attitude of each spacecraft is well-known; (v) All spacecraft are equipped with DBS and TRX; thus, each one of them can localize others; (vi) The standard deviation of the process noise is $10^{-7} \mathrm{~km} / \mathrm{s}^{2}$; (vii) The standard deviation of WLPS measurement noises are 1 meter in TOA and 0.001 degrees in DOA; and (viii) The WLPS transmits its signal at the frequency of $0.1 \mathrm{~Hz}$ ( $\delta t=10$ seconds). Finally, in computing $\lambda$ using Eq. (4.8), the reciprocal condition number [96] of the matrix $D P^{+} D^{T}$ is checked. If the reciprocal condition number is lower than $10^{-14}$, then $R_{d}$ is set to be $10^{-18}$.

Two different scenarios are simulated. The first is a single spacecraft in a Low Earth Orbit (LEO). Measurements are assumed available from a ground radar. The second scenario is for a four-spacecraft formation. Each case study listed in Section 4.3 will be simulated for two different orbit sizes: a semimajor of $7000 \mathrm{~km}$ and a semimajor of $11000 \mathrm{~km}$. All spacecraft in a formation are assumed to have the same semimajor axis. The initial anomaly for all spacecraft is 0 degrees. The spacecraft formation initial configuration for all cases is shown in Table 4.1. The configuration shown in Table 4.1 maintains the minimum distance between any two spacecraft at the range of $11 \mathrm{~km}$ to $50 \mathrm{~km}$, and the maximum distance between any two spacecraft in the formation at the range of $12 \mathrm{~km}$ to 
$110 \mathrm{~km}$.

\subsubsection{Absolute Position Estimation with Radar Tracking}

In this section, we assume that a single spacecraft is tracked by a radar system. The constraint presented in Section 4.3 is applied with respect to the three cases, circular true orbit, known $\Delta t_{p}$ and $\Delta t_{a}$, and unknown $\Delta t_{p}$ and $\Delta t_{a}$, as discussed in Section 4.3.

Spacecraft number 1, in Table 4.1, is simulated at the semimajor axis of $7000 \mathrm{~km}$, and eccentricity of 0.05 for non-circular orbit cases.

The radar tracking system measures the spacecraft absolute range, azimuth, and elevation angles. The noise standard deviation of the absolute range measurement is assumed to be $0.1 \mathrm{~km}$, and the noise standard deviation of each of the azimuth and elevation angles measurements is assumed to be 0.01 degrees [97]. The sensitivity matrix for the radar tracking system is similar to that in Eq. (2.13), where $C_{i}$ is an identity matrix, and $\mathbf{r}_{i j} \equiv \mathbf{r}_{i}$. Figure 4.4(a) shows the RMSE of the EKF estimation for the absolute position, versus time. The results show that after 150 minutes of the estimation process, the EKF RMSE falls below a threshold. The average RMSE of EKF, $\bar{\zeta}_{E K F}$ is 22.24 meters. Note that the standard EKF corresponds to the case of $\alpha=\beta=0$. If the standard CKF is implemented $(\alpha=\beta=1)$, the resulting average $\mathrm{RMSE}$ is $\bar{\zeta}_{C K F}=30$ meters.

To explain the impact of introducing the $\alpha$ and $\beta$ parameters in the CKF estimation on the accuracy of estimation, the PERF is computed for various values of $\alpha$ and $\beta$, as shown in figure 4.4(b) for case $\mathrm{C}$. When including $\alpha$ and $\beta$ in the CKF, the estimation accuracy improves in most of the regions of the two parameters. The CKF estimation is better than the EKF estimation because the CKF incorporates additional information into the estimation process. Specifically, the CKF incorporates the fact that at perigee/apogee the rate of change of the range vanishes. To better see this accuracy improvement, consider figures 4.4(c) and 4.4(d). The PERF is plotted at a constant $\beta=0.4$ for all values of $\alpha$, and is plotted for various values of $\beta$ at a constant $\alpha=0.4$. It can be seen that for elliptic orbits, the improvement ranges from $3 \%$ to $5 \%$, depending on the values of $\alpha$ and $\beta$. From figure 4.4, we can also conclude that $\beta$ is best below 0.8 and that $\alpha$ variations does not affect the PERF significantly.

Both EKF and CKF require a certain estimation period to achieve the steady state. Figure 


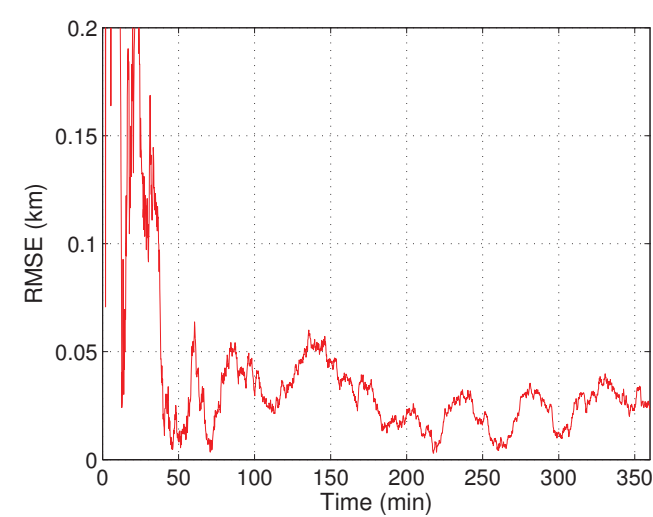

(a) EKF's absolute position RMSE

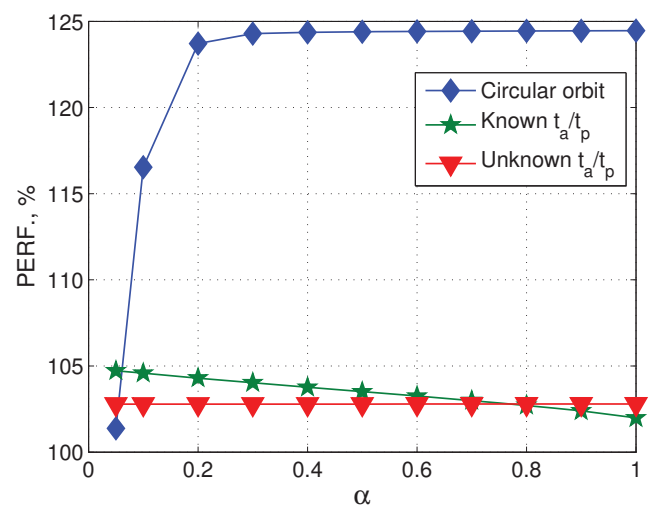

(c) Performance ratio comparison at $\beta=0.4$

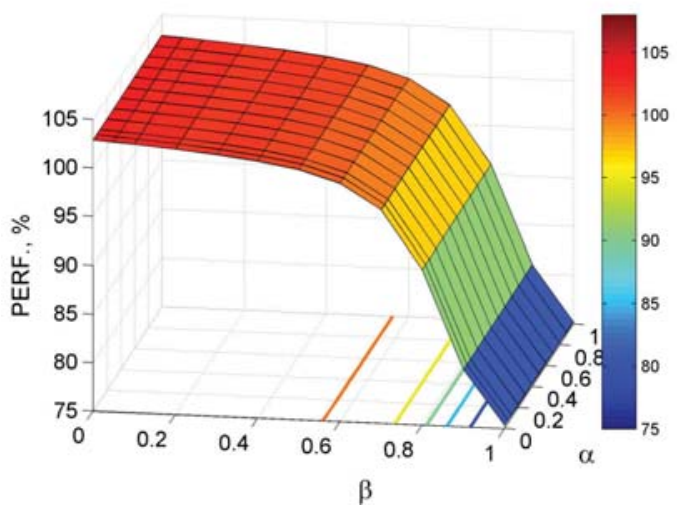

(b) $3 \mathrm{D}$ plot for case $\mathrm{C}$ (unknown $\Delta t_{p}$ and $\Delta t_{a}$ )

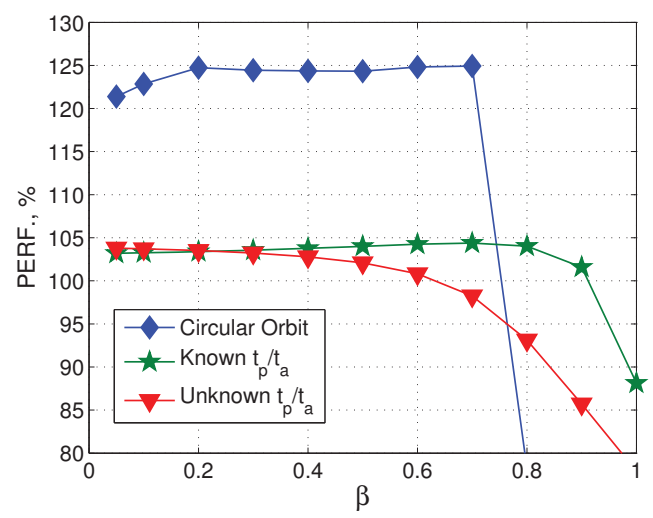

(d) Performance ratio comparison at $\alpha=0.4$

Figure 4.4: $\mathrm{CKF}$ and EKF estimation performance comparison.

4.4(a) shows that the EKF requires at least 100 minutes (about one orbital period) before the RMSE converges to steady state. The time required by the CKF to achieve the steady state is about the same as that of the EKF.

The results also show that the circular orbit has higher PERF compared to the two other cases. Implementing the CKF in a circular orbit improves the estimation accuracy by about $25 \%$ (more than 5 meters) compared to the EKF. This is because the constraint is applied at all points in the circular orbit. In addition, the results also show that the performance improvement is higher if both $\Delta t_{a}$ and $\Delta t_{p}$ are known for the elliptic orbit case, when $\alpha<0.8$. 
Table 4.2

Asbsolute Position Estimation's Average RMSE of EKF.

\begin{tabular}{|cccc|}
\hline Semimajor axis $(\mathrm{km})$ & Orbit Shape & Initial Condition & Average RMSE (meter) \\
\hline 7000 & Circular & well-known & 3.097 \\
11000 & Circular & well-known & 5.195 \\
7000 & Elliptic & well-known & 6.080 \\
11000 & Elliptic & well-known & 22.358 \\
7000 & Elliptic & small error & 105.592 \\
11000 & Elliptic & small error & 172.740 \\
\hline
\end{tabular}

\subsubsection{Scenario two - formation flying}

In this section, a four-spacecraft formation is assumed, as shown in Table 4.1. The three cases presented in Section 4.3 are studied in this section. In addition, two types of initial conditions are investigated for the case presented in Section 4.3.3. As benchmark results, the average RMSE when implementing the EKF for the three case studies are summarized in Table 4.2. In Table 4.2, two cases are simulated with small errors in initial conditions. This error randomly generated in the initial position and velocity vectors. The standard deviation for the initial position error is $1 \mathrm{~km}$, and for the initial velocity error is $1 \mathrm{~ms}^{-1}$. The effect of implementing the CKF with various values for $\alpha$ and $\beta$ are detailed below. It should be noted that since we estimate the absolute positions using only relative position measurements, the estimation accuracy will be altitude dependent, whether we use EKF or CKF. This is because the motion dynamics cause the relative positions between the spacecraft to change differently at different altitudes.

\subsubsection{Formation Flying in a circular orbit}

Figures 4.5(a) and 4.5(b) show the PERF for two values of the semimajor axis: $7000 \mathrm{~km}$ and $11000 \mathrm{~km}$. Both figures show that the CKF performs better than the EKF for most of the ranges of $\alpha$ and $\beta$, in terms of estimation accuracy. The estimation accuracy improved by up to $500 \%$ at $a=7000 \mathrm{~km}$, and up to $350 \%$ at $a=11000 \mathrm{~km}$. Table 4.2 shows that the EKF has higher average RMSE at the higher altitude, 3 meters at $a=7000 \mathrm{~km}$ compared to 5 meters at $a=11000 \mathrm{~km}$. Hence, the CKF implementation improves the estimation accuracy to about 1.5 meters for both altitudes, when $0.5<\alpha<1.0$ and $0.4<\beta<0.7$. It is to be noted that if $\beta$ is high and $\alpha$ is low, e.g., $\beta>0.8$ and $\alpha<0.2$, the performance 


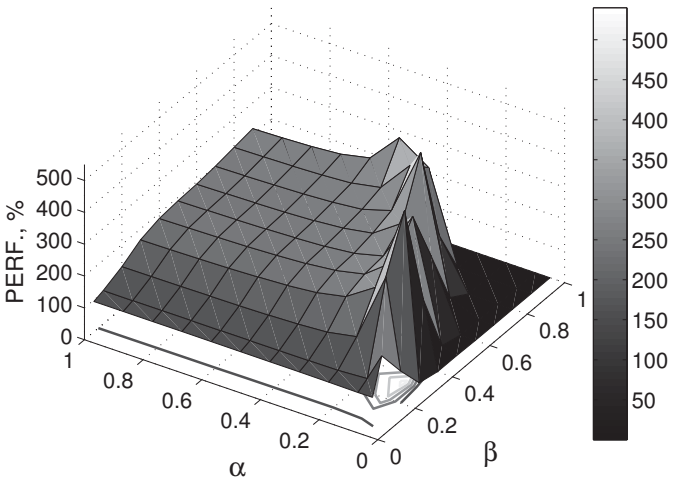

(a) $a=7000 \mathrm{~km}$.

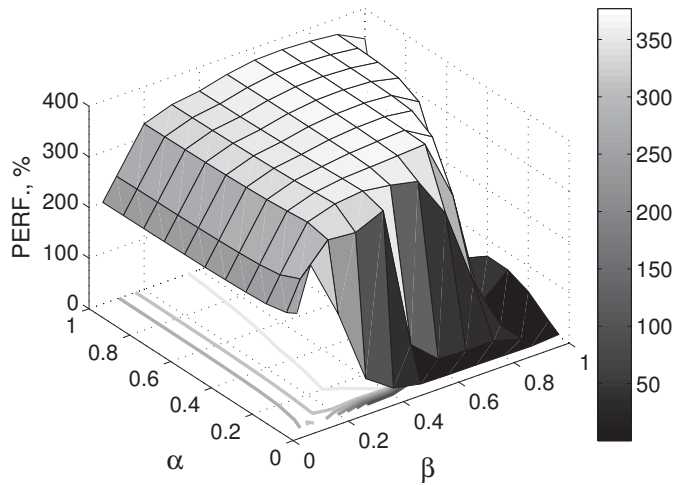

(b) $a=11000 \mathrm{~km}$.

Figure 4.5: Estimation accuracy of CKF relative to that of EKF, for circular orbits

becomes worse than the EKF estimation performance. A higher value for $\beta$ means more confidence in the constraint update; and hence causes the covariance to converge faster. This result confirms that the estimation performance becomes worse if the covariance converges faster than the state, causing the estimated state's error to go outside the covariance boundary.

\subsubsection{SFF in eccentric orbit - known perigee/apogee times}

In this section, the eccentricity for all the spacecraft in the formation is 0.05 . We assume that both $\Delta t_{p}$ and $\Delta t_{a}$ for each spacecraft are known at all times. Given the semimajor axis, $a$, of each spacecraft in the formation, the orbital period of the spacecraft is:

$$
T=2 \pi \sqrt{\frac{a^{3}}{\mu}}
$$

where $\mu$ is the Earth gravitational constant.

If the spacecraft's initial anomaly is known, the time required by each spacecraft to arrive at either apogee or perigee position can be determined. For example, for the spacecraft with initial anomaly of 0 degree, it will arrive at apogee position at $t=\frac{1}{2} T$, and perigee position at $t=T$. The algorithm presented in Section 4.3.2 is implemented for the constraint estimation. 


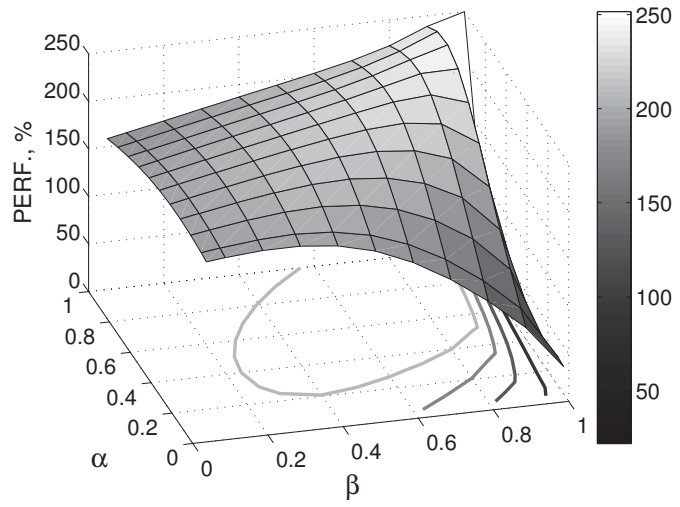

(a) $a=7000 \mathrm{~km}$.

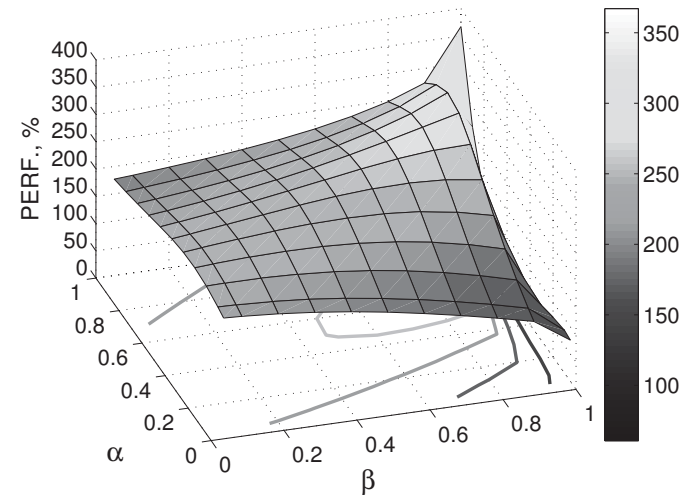

(b) $a=11000 \mathrm{~km}$.

Figure 4.6: Estimation accuracy of CKF relative to that of EKF, for eccentric orbits with known perigee/apogee times

Figures 4.6(a) and 4.6(b) compare the PERF for different values of $\alpha$ and $\beta$, for semimajor axis values of $7000 \mathrm{~km}$ and $11000 \mathrm{~km}$. Both figures show that the CKF improves the estimation performance at any $\alpha$ and $\beta$ combination except when $\alpha$ is very low and $\beta$ is high, i.e., $\alpha<0.2$ and $\beta>0.9$. This is in agreement with the discussion in section 4.4.2.1. The results also show that the estimation accuracy is guaranteed to be improved at any $\alpha$ value with $\beta=0.8$. Also, by comparing the results in both Figures 4.6(a) and 4.6(b), higher altitude results in higher accuracy improvement. Recall the EKF results listed in Table 4.2; and using the results from figure 4.6, it can be shown that the CKF estimation improves the estimation accuracy to 6 meters at a semimajor axis of $11000 \mathrm{~km}$, and to 3 meters at a semimajor axis of $7000 \mathrm{~km}$.

\subsubsection{FF in eccentric orbit - unknown perigee/apogee time}

In this section, the spacecraft formation configuration is similar to Section 4.4.2.2. It is assumed that the $\Delta t_{p}$ and $\Delta t_{a}$ for all spacecraft are unknown. Therefore, the procedure to determine $\Delta t_{a}$ and $\Delta t_{p}$ presented in Section 4.3.3, is implemented.

Figures 4.7(a) and 4.7(b) compare the PERF of different values of $\alpha$ and $\beta$, for semimajor axis values of $7000 \mathrm{~km}$ and $11000 \mathrm{~km}$. Both figures show that the $\alpha$ has less impact on the performance improvement, as opposed to $\beta$. Both Figures 4.7(a) and 4.7(b) emphasize the previous remarks that $\beta$ is best below 0.8 . Table 4.2 shows that the EKF accuracy 


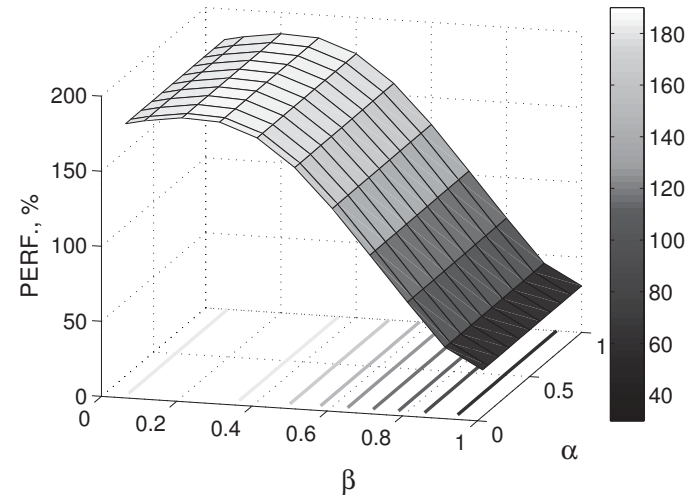

(a) $a=7000 \mathrm{~km}$.

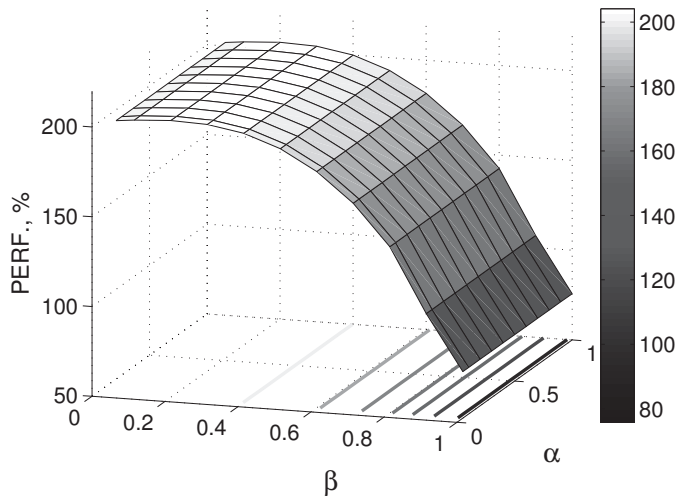

(b) $a=11000 \mathrm{~km}$.

Figure 4.7: Estimation accuracy of CKF relative to that of EKF, for eccentric orbits with unknown perigee/apogee times

decreases when the altitude of SFF increases. Therefore, Figure 4.7(b) implies that implementing the CKF has more impact at higher altitudes.

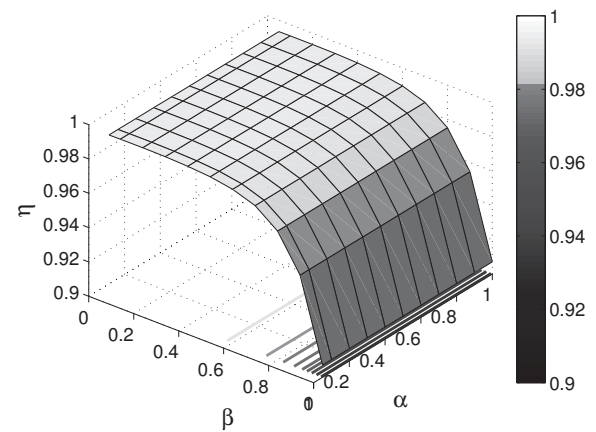

Figure 4.8: Estimation accuracy of CKF relative to that of EKF, for eccentric orbits with unknown perigee/apogee times and initial guess errors, for $a=11000 \mathrm{~km}$.

Figure 4.8 shows the PERF when there are small errors in the initial guess of the position and velocity vectors. Table 4.2 shows that the EKF has degraded performance when the initial conditions are not perfectly known; the average RMSE is at least 10 to 20 times that of the case when the initial conditions are well known. However, Figure 4.8 shows that implementing the apogee and perigee constraints into the estimation does improve the estimation accuracy. The overall results agree with the previous case studies, in which the $\alpha$ has less impact on the CKF implementation, and very high $\beta$ values degrade the CKF's accuracy performance. 
As mentioned earlier, the EKF accuracy depends on the altitude. On the other hand, the $\mathrm{CKF}$ incorporates the constraints at perigee and apogee, and hence provides direct information about the orbit size and hence the absolute range. So, the CKF estimation accuracy in this chapter tends to be independent from the altitude. The higher impact of the CKF, shown in the PERF figures, is because the EKF accuracy is worse at higher altitudes (the PERF is a ration of EKF accuracy to CKF accuracy). 


\section{Chapter 5}

\section{Spacecraft Formation Relative Attitude Determination}




\subsection{Introduction}

Relative Attitude Determination (RAD) continues to receive a great deal of interest from researchers. ${ }^{*}$ Missions that require RAD include the spacecraft docking missions, chaser and target space missions [8], and clusters that perform their tasks in specific formations, such as Proba-3 [9] and LISA Pathfinder's missions [10, 11]. In these missions, the spacecraft are often required to maintain a specific orientation with respect to each other. The relative attitude determination between spacecraft is a fundamental task in these types of missions.

The relative attitudes between spacecraft can be calculated if the absolute attitude of each spacecraft is known. However, the determination of the absolute attitude for each

spacecraft requires a complete set of attitude sensors onboard each spacecraft [89]. On the other hand, the direct determination of relative attitudes require less hardware on each spacecraft. One of the fundamental approaches, to directly determine the relative attitudes between spacecraft, is to use the measurements for the relative directions between spacecraft for relative attitudes determination. From a cost prospective, the computational complexity in computing relative attitudes directly is less than that of computing them through the absolute attitudes [98]. Thus, if absolute attitudes are not needed, then measuring relative attitudes directly offers a lower cost advantage. The determination of relative attitudes between spacecraft can also be used to calculate the absolute attitudes, if the absolute attitude for one spacecraft is known.

The literature includes descriptions for several sensors that are capable of providing measurements for relative attitudes and relative positions. The Autonomous Formation Flying (AFF) sensor of the Deep Space program [3] works in a similar way to that of the Global Positioning System (GPS) [49-51, 99]. The Vision Based Navigation System (VISNAV) has been introduced as a candidate for relative attitude and relative position sensing [7, 49]. The measurements of the VISNAV along with the system's dynamic model can be integrated in an estimator, such as the Extended Kalman Filter (EKF), to estimate the relative attitudes, as explained in reference [7]. However, the stability of estimation, in this configuration of VISNAV sensor and EKF, is not guaranteed [100]. The estimator's stability is affected by several factors such as the initial condition errors, the unexpected gyro drift rate, and the non line-of-sight measurements [7, 22, 101].

${ }^{*}$ The material contained in this chapter was previously published in the 2010 IEEE Aerospace conference proceeding. 


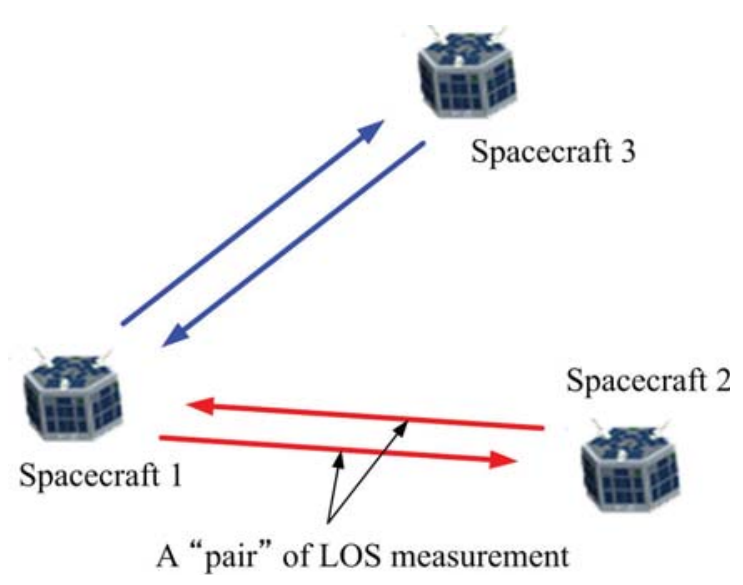

Figure 5.1: TRIAD method requires two pairs of LOS measurements.

Moreover, in some Spacecraft Formation Flying (SFF) missions, such as the LISA pathfinder mission, where each spacecraft is located at about $5 \times 10^{6} \mathrm{~km}$ away from the other spacecraft [102], a small error in the Line-of-Sight (LOS) measurement results in a large error in the relative position calculation. This instability issue in the estimator lowers the reliability of the method, in this case, given that it is hard to predict when this instability would occur.

Attitude determination methods, on the other hand, offer an attractive approach. A fundamental three-axis attitude determination method is the TRIAD method, developed by Shuster [103, 104], for absolute attitude determination. In general, attitude determination methods can be categorized as optimal and non=optimal attitude determination methods. Optimal methods determine the attitude by minimizing the attitude error that is a function of the measurements errors [105-107]. Non-optimal methods, on the other hand, determine the attitude geometrically from the measurements sets. The TRIAD [103], CONES intersection [108], and EULER-2 [109] methods are non-optimal methods; where Quaternion Estimator (QUEST) [110], Fast Optimal Attitude Matrix (FOAM) [111] and Estimator of the Optimal Quaternion (ESOQ) [112] are optimal attitude determination methods.

Consider a formation of three spacecraft as shown in figure 5.1. TRIAD method can be used to find the relative attitude between two spacecraft (say spacecraft 1 and spacecraft 2) only if the relative attitude of the third spacecraft (spacecraft 3 ) with respect to spacecraft 1 (or spacecraft 2) is known. The two LOS measurements between two spacecraft (i.e., spacecraft 1 measured by spacecraft 2 , and spacecraft 2 measured by spacecraft 1 ), are 
called a pair of measurements. The TRIAD method requires two "pairs" of measurements (see figure 5.1) expressed in two reference frames. The two reference frames are attached to the two spacecraft (Spacecraft 1 and 2) between which we intend to determine the relative attitude between them.

Ref. [12] introduced a RAD method for a three-vehicle formation, using all three pairs of measurements between the three spacecraft. The relative attitude between two spacecraft is determined using an angle and a vector as the constraints. Then, the TRIAD method is used to find the relative attitude between the other two spacecraft. Althought the previous work has shown that the covariance singularity due to the co-planar may be encountered [13], Ref. [12] showed that the proposed method does not process any singularity issue.

In this chapter, we present an alternative RAD method that avoids the coplanarity issue. we consider the case of a three-spacecraft formation, where each spacecraft is capable of measuring the directions of the other two. The proposed method uses one pair of measurements, along with two measurements from the other two pairs, to determine the relative attitude between two spacecraft in a three-spacecraft formation. A fifth single measurement may be added to determine the relative attitude between the other two spacecraft. The proposed method benefits from the concept of the TRIAD method of absolute attitude determination, through the introduction of an intermediate reference frame, as detailed in Section 2.

Section 5.2 presents the derivation for the proposed relative attitude determination method and covariance analysis for the parallel case. Section 5.3 presents the derivation of relative attitude determination method and its covariance analysis for the non-parallel case. The simulation is set up and discussed in Section 5.4.

\subsection{Relative Attitude Determination}

In this section, the algorithm to determine the relative attitude is presented. Figure 5.2 shows three spacecraft flying in a formation. Each spacecraft provides the line-of-sight (LOS) measurement vectors of the other two in its respective coordinate reference frame. We define the relative position of the $j^{\text {th }}$ spacecraft observed by the $i^{\text {th }}$ spacecraft as the vector, $\mathbf{r}_{i j}^{k}$; where the superscript $k$ designates that the vector components are in the $k^{t h}$ frame. 


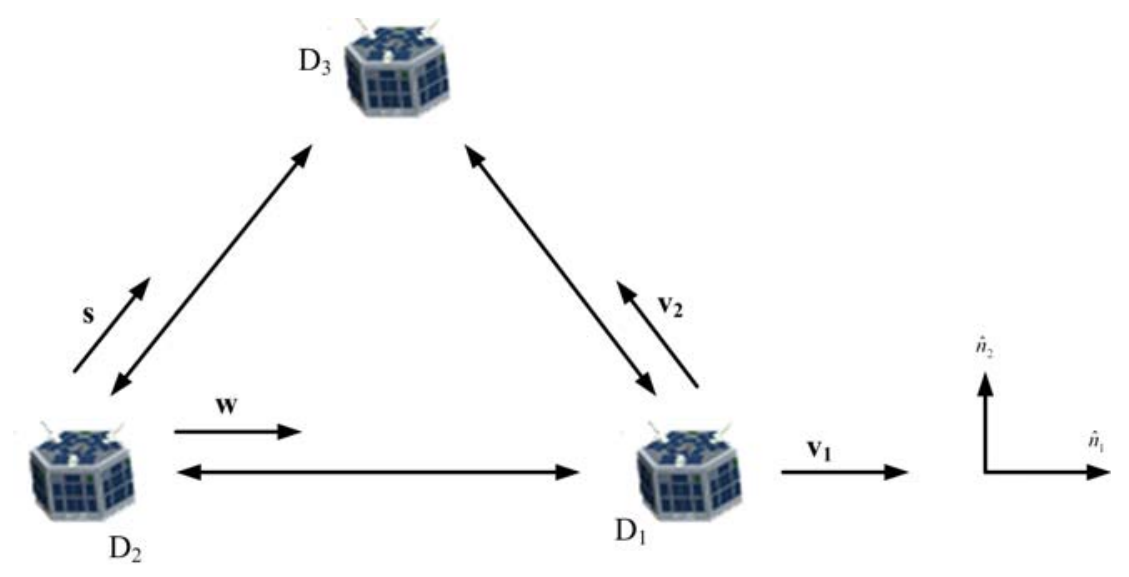

Figure 5.2: Measurements in three spacecraft formation.

For simplicity, assume that it is required to compute only the relative attitudes between spacecraft; not the relative positions. Hence, only the measurements for the directions between spacecraft are needed. The determination of the relative positions may require the measurements of relative position vectors. The direction of the $j^{t h}$ spacecraft observed by the $i^{t h}$ spacecraft may be represented by a unit vector in the direction of $\mathbf{r}_{i j}^{k}$,

$$
\mathbf{p}_{i j}^{k}=\frac{\mathbf{r}_{i j}^{k}}{\left\|\mathbf{r}_{i j}^{k}\right\|}
$$

where the subscript $i j$ represents the observation of the $D_{j}$ spacecraft by the $D_{i}$ spacecraft, and the superscript $k$ indicates that the observation vector is expressed in the $D_{k}$ reference frame. Note that $\mathbf{p}_{i j}^{k}=-\mathbf{p}_{j i}^{k}$ only if both observation vectors are measured in same reference frame, $k$.

As shown in figure 5.2, we define the following unit vectors: $\mathbf{w}=\mathbf{p}_{21}^{2}, \mathbf{s}=\mathbf{p}_{23}^{2}, \mathbf{v}_{2}=\mathbf{p}_{13}^{1}$, and $\mathbf{v}_{1}=-\mathbf{p}_{12}^{1}=\mathbf{p}_{21}^{1}$. Note that the observation vector, $\mathbf{v}_{1}$ is the observation of $D_{1}$ spacecraft by $D_{2}$ spacecraft, expressed in the $D_{1}$ spacecraft reference frame. Next, we consider the relative attitude determination between the $D_{2}$ and $D_{1}$ spacecraft. As seen in Figure 5.2, an intermediate reference frame $(\mathrm{N})$ is introduced, which is defined by three unit vectors $\hat{\mathbf{n}}_{1}, \hat{\mathbf{n}}_{2}$ and $\hat{\mathbf{n}}_{3}$. The unit vector $\hat{\mathbf{n}}_{1}$ is directed from $D_{2}$ to $D_{1}$, and so:

$$
\mathbf{v}_{1}=A_{1} \hat{\mathbf{n}}_{1}
$$

where $A_{1}$ is the relative attitude matrix, which is also known as the Direction Cosine Matrix [74], between the intermediate frame (N) and the frame $D_{1}$. 
The unit vector $\hat{\mathbf{n}}_{3}$ is normal to the plane, defined by $D_{1}, D_{2}$, and $D_{3}$ spacecraft. Also, $\hat{\mathbf{n}}_{3}=\hat{\mathbf{n}}_{1} \times \hat{\mathbf{n}}_{2}$, and is given by:

$$
\frac{\mathbf{v}_{1} \times \mathbf{v}_{2}}{\left\|\mathbf{v}_{1} \times \mathbf{v}_{2}\right\|}=A_{1} \hat{\mathbf{n}}_{3}
$$

Finally, $\hat{\mathbf{n}}_{2}$ is perpendicular to $\hat{\mathbf{n}}_{1}$ and lies in the plane defined by $D_{1}, D_{2}$ and $D_{3}$ spacecraft. The $\hat{\mathbf{n}}_{2}$ can be obtained using the cross product between $\left(\mathbf{v}_{1} \times \mathbf{v}_{2}\right)$ and $\mathbf{v}_{1}$, which can be written as:

$$
\frac{\mathbf{v}_{2}-\left(\mathbf{v}_{2}^{T} \mathbf{v}_{1}\right) \mathbf{v}_{1}}{\left\|\mathbf{v}_{2}-\left(\mathbf{v}_{2}^{T} \mathbf{v}_{1}\right) \mathbf{v}_{1}\right\|}=A_{1} \hat{\mathbf{n}}_{2}
$$

Using equations (5.2) to (5.4), the relative attitude matrix between the intermediate frame (N) and the frame $D_{1}$ is:

$$
A_{1}=\left[\begin{array}{lll}
\mathbf{v}_{1} & \frac{\mathbf{v}_{2}-\left(\mathbf{v}_{2}^{T} \mathbf{v}_{1}\right) \mathbf{v}_{1}}{\left\|\mathbf{v}_{2}-\left(\mathbf{v}_{2}^{T} \mathbf{v}_{1}\right) \mathbf{v}_{1}\right\|} & \frac{\mathbf{v}_{1} \times \mathbf{v}_{2}}{\left\|\mathbf{v}_{1} \times \mathbf{v}_{2}\right\|}
\end{array}\right]
$$

The relative attitude matrix between the intermediate frame $(\mathrm{N})$ and the frame $D_{2}$ may be constructed in a similar way to $A_{1}$ :

$$
A_{2}=\left[\begin{array}{lll}
\mathbf{w} & \frac{\mathbf{s}-\left(\mathbf{s}^{T} \mathbf{w}\right) \mathbf{w}}{\left\|\mathbf{s}-\left(\mathbf{s}^{T} \mathbf{w}\right) \mathbf{w}\right\|} & \frac{\mathbf{w} \times \mathbf{s}}{\|\mathbf{w} \times \mathbf{s}\|}
\end{array}\right]
$$

where,

$$
\begin{gathered}
\mathbf{w}=A_{2} \hat{\mathbf{n}}_{1} \\
\frac{\mathbf{s}-\left(\mathbf{s}^{T} \mathbf{w}\right) \mathbf{w}}{\left\|\mathbf{s}-\left(\mathbf{s}^{T} \mathbf{w}\right) \mathbf{w}\right\|}=A_{2} \hat{\mathbf{n}}_{2} \\
\frac{\mathbf{w} \times \mathbf{s}}{\|\mathbf{w} \times \mathbf{s}\|}=A_{2} \hat{\mathbf{n}}_{3}
\end{gathered}
$$

The inverse of an attitude matrix is its transpose [74], that is $\left(A_{k}\right)^{-1}=\left(A_{k}\right)^{T}$. Given that $\mathbf{v}_{1}=A_{1} \hat{\mathbf{n}}_{1}$, we may also write:

$$
\hat{\mathbf{n}}_{1}=A_{1}^{T} \mathbf{v}_{1}
$$

Substituting equation (5.10) into equation (5.7), we obtain:

$$
\mathbf{w}=A_{2} A_{1}^{T} \mathbf{v}_{1} \equiv A_{1}^{2} \mathbf{v}_{1}
$$


where $A_{1}^{2}$ is the relative attitude of $D_{1}$ frame relative to $D_{2}$ frame:

$$
A_{1}^{2}=A_{2} A_{1}^{T}
$$

Also, it is noted here that, $A_{j}^{i}=\left(A_{i}^{j}\right)^{T}$, and so

$$
\mathbf{v}_{1}=\left(A_{1}^{2}\right)^{T} \mathbf{w} \equiv A_{2}^{1} \mathbf{w}
$$

The relative attitude matrix between $D_{1}$ and $D_{3}$ (or between $D_{2}$ and $D_{3}$ ) can be obtained in a similar fashion, if we have additional measurements between $D_{3}$ and $D_{1}$ (or between $D_{3}$ and $\left.D_{2}\right)$.

TRIAD method was originally developed to determine the absolute attitude of spacecraft. The TRIAD method can also be used for spacecraft formation relative attitude determination. If one of the relative attitudes between spacecraft is known, TRIAD can be used to determine the other two relative attitudes. Assuming that the relative attitude between $D_{1}$ and $D_{3}, A_{3}^{1}$, is known, then the TRIAD method may be applied using two "pairs" of LOS measurements, $\left(\mathbf{p}_{12}^{1}, \mathbf{p}_{21}^{2}\right)$, and $\left(\mathbf{p}_{23}^{2}, \mathbf{p}_{32}^{3}\right)$, to determine $A_{1}^{2}$ and $A_{2}^{3}$. In the next section, the covariance associated with this RAD method will be developed. The covariance analysis will also show that this RAD method does not have any singularity problem.

\subsubsection{Covariance Analysis}

Here, the covariance of relative attitude determination error between any two spacecraft in the formation is derived. The covariance, or often referred to as variance, is expressed in matrix format. Due to measurements' noises, the attitude determination process has a certain degree of error. This error falls within the three sigma covariance boundary [22]. In addition to estimating the error boundary in attitude determination, the covariance analysis in this section will address the singularity issue encountered when all measurements are in the same plane, as detailed in Ref. [12]. We will show, in this section, that the RAD method developed in this chapter avoids this singularity issue. Let the true state vector be $\mathbf{x}$, and the corresponding estimated state vector be $\hat{\mathbf{x}}$. The 
covariance of the estimated state vector is defined as [22]:

$$
P_{1}^{2}=E\left\{(\hat{\mathbf{x}}-\mathbf{x})(\hat{\mathbf{x}}-\mathbf{x})^{T}\right\}
$$

where $E\{$.$\} denotes the expectation.$

The true state is never known. To obtain a form for the covariance of the estimated state vector, we set the covariance equal to the lower bound of Cramer Rao inequality [12]:

$$
P_{1}^{2} \equiv E\left\{(\hat{\mathbf{x}}-\mathbf{x})(\hat{\mathbf{x}}-\mathbf{x})^{T}\right\}=F^{-1}
$$

where $F$ is the Fisher Information Matrix [22, 113], which is given by:

$$
F=-E\left\{\frac{\partial}{\partial \Delta \mathbf{x} \partial \Delta \mathbf{x}} J(\Delta \mathbf{x})\right\}
$$

where $J(\Delta \mathbf{x})$ is the loss function (or error function), and $\Delta \mathbf{x} \equiv \hat{\mathbf{x}}-\mathbf{x}$.

Equations (5.15) and (5.16) show that the lower bound of the covariance is equivalent to the inverse of the Fisher Information Matrix. The relative attitude matrix between any two spacecraft, derived in (5.5) to (5.12), will be used to evaluate the error function in (5.16). Combining both equations (5.5) and (5.6):

$$
\begin{aligned}
\mathbf{w}_{1} & =A_{1}^{2} \mathbf{v}_{1} \\
\frac{\mathbf{s}-\left(\mathbf{s}^{T} \mathbf{w}_{1}\right) \mathbf{w}_{1}}{\left\|\mathbf{s}-\left(\mathbf{s}^{T} \mathbf{w}_{1}\right) \mathbf{w}_{1}\right\|} & =A_{1}^{2} \frac{\mathbf{v}_{2}-\left(\mathbf{v}_{2}^{T} \mathbf{v}_{1}\right) \mathbf{v}_{1}}{\left\|\mathbf{v}_{2}-\left(\mathbf{v}_{2}^{T} \mathbf{v}_{1}\right) \mathbf{v}_{1}\right\|} \\
\frac{\mathbf{w}_{1} \times \mathbf{s}}{\left\|\mathbf{w}_{1} \times \mathbf{s}\right\|} & =A_{1}^{2} \frac{\mathbf{v}_{1} \times \mathbf{v}_{2}}{\left\|\mathbf{v}_{1} \times \mathbf{v}_{2}\right\|}
\end{aligned}
$$

where $A_{1}^{2}$ denotes the estimated relative attitude of spacecraft $D_{2}$ with respect to spacecraft $D_{1}$. 
Let:

$$
\begin{gathered}
\mathbf{w}_{3}=\frac{\mathbf{s}-\left(\mathbf{s}^{T} \mathbf{w}_{1}\right) \mathbf{w}_{1}}{\left\|\mathbf{s}-\left(\mathbf{s}^{T} \mathbf{w}_{1}\right) \mathbf{w}_{1}\right\|} \\
\mathbf{w}_{4}=\frac{\mathbf{w}_{1} \times \mathbf{s}}{\left\|\mathbf{w}_{1} \times \mathbf{s}\right\|} \\
\mathbf{v}_{3}=\frac{\mathbf{v}_{2}-\left(\mathbf{v}_{2}^{T} \mathbf{v}_{1}\right) \mathbf{v}_{1}}{\left\|\mathbf{v}_{2}-\left(\mathbf{v}_{2}^{T} \mathbf{v}_{1}\right) \mathbf{v}_{1}\right\|} \\
\mathbf{v}_{4}=\frac{\mathbf{v}_{1} \times \mathbf{v}_{2}}{\left\|\mathbf{v}_{1} \times \mathbf{v}_{2}\right\|}
\end{gathered}
$$

Then we can write equations (5.17) to (5.19) as:

$$
\begin{aligned}
& \mathbf{w}_{1}=A_{1}^{2} \mathbf{v}_{1} \\
& \mathbf{w}_{3}=A_{1}^{2} \mathbf{v}_{3} \\
& \mathbf{w}_{4}=A_{1}^{2} \mathbf{v}_{4}
\end{aligned}
$$

The vectors $\mathbf{w}_{1}, \mathbf{w}_{3}$, and $\mathbf{w}_{4}$ align with the coordinates of the body-fixed reference frame on $D_{2}$ with respect to the intermediate frame (N). Similarly, the vectors $\mathbf{v}_{1}, \mathbf{v}_{3}$, and $\mathbf{v}_{4}$ align with the coordinates of the body-fixed reference frame on $D_{1}$ with respect to the intermediate frame $(\mathrm{N})$. If the measurements have any error or noise (which is usually the case), equations (5.24) to (5.26) will not be satisfied. So, we construct the error function as follows:

$$
\begin{aligned}
J & =\frac{1}{2}\left(\mathbf{w}_{1}-A_{1}^{2} \mathbf{v}_{1}\right)^{T} R_{1}^{-1}\left(\mathbf{w}_{1}-A_{1}^{2} \mathbf{v}_{1}\right)+\frac{1}{2}\left(\mathbf{w}_{3}-A_{1}^{2} \mathbf{v}_{3}\right)^{T} R_{3}^{-1}\left(\mathbf{w}_{3}-A_{1}^{2} \mathbf{v}_{3}\right) \\
& +\frac{1}{2}\left(\mathbf{w}_{4}-A_{1}^{2} \mathbf{v}_{4}\right)^{T} R_{4}^{-1}\left(\mathbf{w}_{4}-A_{1}^{2} \mathbf{v}_{4}\right)
\end{aligned}
$$

where $R_{1}, R_{3}$ and $R_{4}$ are the measurements covariances, which will be computed in section 5.2.2.

Assume that the relative attitude errors are small error angles. Define $\delta \alpha_{i}^{j}$ to be the small error angle vector in the relative attitude of $D_{j}$ with respect to $D_{i}$. The estimated relative attitude is expressed in terms of the true relative attitude and $\delta \alpha_{i}^{j}$ as $[12,22,80]$ :

$$
A_{1}^{2}=\left(I_{3 \times 3}-\left[\delta \alpha_{1}^{2} \times\right]\right) A_{1_{t}}^{2}
$$


The operator, $[\mathbf{a} \times]$ denotes the cross product matrix $[22,74]$ of a vector $\mathbf{a}=\left[\begin{array}{lll}a_{1} & a_{2} & a_{3}\end{array}\right]^{T}:$

$$
[\mathbf{a} \times]=\left[\begin{array}{ccc}
0 & -a_{3} & a_{2} \\
a_{3} & 0 & -a_{1} \\
-a_{2} & a_{1} & 0
\end{array}\right]
$$

Substituting equation (5.28) into equation (5.27), with $\Delta \mathbf{x} \equiv \delta \alpha_{1}^{2}$, the loss function becomes:

$$
\begin{aligned}
J\left(\delta \alpha_{1}^{2}\right) & =\frac{1}{2} \delta \alpha_{1}^{2^{T}}\left[A_{1_{t}}^{2} \mathbf{v}_{1} \times\right]^{T} R_{1}^{-1}\left[A_{1_{t}}^{2} \mathbf{v}_{1} \times\right] \delta \alpha_{1}^{2}+\frac{1}{2} \delta \alpha_{1}^{2^{T}}\left[A_{1_{t}}^{2} \mathbf{v}_{3} \times\right]^{T} R_{2}^{-1}\left[A_{1_{t}}^{2} \mathbf{v}_{3} \times\right] \delta \alpha_{1}^{2} \\
& +\frac{1}{2} \delta \alpha_{1}^{2^{T}}\left[A_{1_{t}}^{2} \mathbf{v}_{4} \times\right]^{T} R_{3}^{-1}\left[A_{1_{t}}^{2} \mathbf{v}_{4} \times\right] \delta \alpha_{1}^{2}
\end{aligned}
$$

The error covariance matrix of equation (5.14) is determined by taking the second order partial derivative of $J$ with respect to $\delta \alpha_{1}^{2}$ :

$$
P_{1}^{2}=\left(\left[A_{1_{t}}^{2} \mathbf{v}_{1} \times\right] R_{1}^{-1}\left[A_{1_{t}}^{2} \mathbf{v}_{1} \times\right]^{T}+\left[A_{1_{t}}^{2} \mathbf{v}_{3} \times\right] R_{2}^{-1}\left[A_{1_{t}}^{2} \mathbf{v}_{3} \times\right]^{T}+\left[A_{1_{t}}^{2} \mathbf{v}_{4} \times\right] R_{3}^{-1}\left[A_{1_{t}}^{2} \mathbf{v}_{4} \times\right]^{T}\right)^{-1}
$$

The Fisher Information Matrix is:

$$
F=\left[A_{1_{t}}^{2} \mathbf{v}_{1} \times\right] R_{1}^{-1}\left[A_{1_{t}}^{2} \mathbf{v}_{1} \times\right]^{T}+\left[A_{1_{t}}^{2} \mathbf{v}_{3} \times\right] R_{2}^{-1}\left[A_{1_{t}}^{2} \mathbf{v}_{3} \times\right]^{T}+\left[A_{1_{t}}^{2} \mathbf{v}_{4} \times\right] R_{3}^{-1}\left[A_{1_{t}}^{2} \mathbf{v}_{4} \times\right]^{T}
$$

The cross product matrix in equation (5.29), for any vector, is always singular. Also $[\mathbf{a} \times][\mathbf{a} \times]^{T}$ is always singular. Thus, if all three observation vectors $\mathbf{p}_{31}^{2}, \mathbf{p}_{32}^{2}$, and $\mathbf{p}_{21}^{2}$ are in the same plane, the Fisher Information Matrix becomes singular.

The vector $\mathbf{v}_{4}$, though, is perpendicular to both vectors $\mathbf{v}_{1}$ and $\mathbf{v}_{3}$ in equation (5.31); so $\mathbf{v}_{1} \neq c_{1} \mathbf{v}_{3}+c_{2} \mathbf{v}_{4}$. The covariance matrix derived in equation (5.31) is always non-singular. The covariance analysis for relative attitude determination between $D_{1}$ and $D_{3}$, and between $D_{2}$ and $D_{3}$ may also be derived in a similar fashion as shown in equation (5.31). 


\subsubsection{Measurement Covariance - Parallel Case}

Here, the measurements covariances associated with equations (5.24) to (5.26) are derived. The derivation follows a similar approach to that shown in Ref. [12] and [80], but using the new RAD method presented in this chapter.

Let a measurement vector be expressed as:

$$
\tilde{\mathbf{p}}_{i}=\mathbf{p}_{i}+v_{i}
$$

where $\mathbf{p}_{i}$ is the true value for the measured quantity, $\tilde{\mathbf{p}}_{i}$ is the measured quantity, and $v_{i}$ denotes the measurement noises of $\mathbf{p}_{i}$.

We assume that there is no correlation between the measurement noises in equation (5.33). The measurement noise covariance, $\Omega\left(\tilde{\mathbf{p}}_{i}\right)$ is:

$$
\Omega\left(\tilde{v}_{i}\right)=E\left\{\tilde{v}_{i} \tilde{v}_{i}^{T}\right\}=\left[\begin{array}{ccc}
\sigma_{x}^{2} & 0 & 0 \\
0 & \sigma_{y}^{2} & 0 \\
0 & 0 & \sigma_{z}^{2}
\end{array}\right]
$$

where $\sigma_{x}, \sigma_{y}$ and $\sigma_{z}$ are the standard deviations of the three components of the measurement noise vector, $v_{i}$.

Equations (5.24) to (5.26) are in the form of $\tilde{\mathbf{p}}_{i}=A \tilde{\mathbf{p}}_{j}$. The measurement noise covariance for equations (5.24) to (5.26) can be written in matrix form as:

$$
R_{i}=E\left\{\left(\tilde{\mathbf{p}}_{i}-A \tilde{\mathbf{p}}_{j}\right)\left(\tilde{\mathbf{p}}_{i}-A \tilde{\mathbf{p}}_{j}\right)^{T}\right\}=\Omega_{i}+A \Omega_{j} A^{T}
$$

Equation (5.35) can be used to find $R_{1}$ directly because the $R_{1}$ is a linear function of the measurement noises. The covariances $R_{3}$ and $R_{4}$ associated with equations (5.18) and (5.19), on the other hand, are not linear functions in the measurement noises. The nonlinearity can be verified by comparing equations (5.25), (5.26), and (5.35). Andrle et al. $[12,80]$ showed how to compute the covariance of the unit vector, $\mathbf{b}$, of an arbitrary noisy measured vector, $\mathbf{r}$ :

$$
\Omega_{\mathbf{b}}=\left(\frac{\partial \mathbf{b}}{\partial \mathbf{r}}\right) \Omega_{\mathbf{r}}\left(\frac{\partial \mathbf{b}}{\partial \mathbf{r}}\right)^{T}
$$


where,

$$
\frac{\partial \mathbf{b}}{\partial \mathbf{r}}=-\|\mathbf{r}\|^{-3}[\mathbf{r} \times]^{2}
$$

In many estimation algorithms, the measurements noise covariance is assumed to be linear additive to simplify the estimation process. However, Ref. [82] shows that the nonlinear noise covariance could be derived without degrading the estimation quality. This method may be applied to the relative attitude determination method presented in this chapter. Assume that the variance of the noise is small, $\sigma^{2}<<1$, then we may conclude that $\sigma^{4} \approx 0$. Define the operator $A \odot B$ as the element-by-element multiplication of the matrices $\mathrm{A}$ and B. Define also $M(\mathbf{b})$ as:

$$
M(\mathbf{b})=\left[\begin{array}{lll}
\mathbf{b} & \mathbf{b} & \mathbf{b}
\end{array}\right]^{T}
$$

Then, the covariance associated with equation (5.18) is:

$$
\begin{aligned}
R_{3} & =E\left\{\left(\mathbf{w}_{3}-A_{1}^{2} \mathbf{v}_{3}\right)\left(\mathbf{w}_{3}-A_{1}^{2} \mathbf{v}_{3}\right)^{T}\right\} \\
& =\left(\frac{\partial \mathbf{w}_{3}}{\partial \mathbf{r}_{1}}\right) \Omega\left(\mathbf{s}-\left(\mathbf{s}^{T} \mathbf{w}_{1}\right) \mathbf{w}_{1}\right)\left(\frac{\partial \mathbf{w}_{3}}{\partial \mathbf{r}_{1}}\right)^{T}+A_{1}^{2}\left(\frac{\partial \mathbf{v}_{3}}{\partial \mathbf{r}_{2}}\right) \Omega\left(\mathbf{v}_{2}-\left(\mathbf{v}_{2}^{T} \mathbf{v}_{1}\right) \mathbf{v}_{1}\right)\left(\frac{\partial \mathbf{v}_{3}}{\partial \mathbf{r}_{2}}\right)^{T} A_{1}^{2^{T}}
\end{aligned}
$$

where the $\mathbf{r}_{1}$ represents $\mathbf{s}-\left(\mathbf{s}^{T} \mathbf{w}_{1}\right) \mathbf{w}_{1}$ and $\mathbf{r}_{2}$ represents $\left(\mathbf{v}_{2}-\left(\mathbf{v}_{2}^{T} \mathbf{v}_{1}\right) \mathbf{v}_{1}\right)$.

Both vectors, $\mathbf{s}-\left(\mathbf{s}^{T} \mathbf{w}_{1}\right) \mathbf{w}_{1}$ and $\left(\mathbf{v}_{2}-\left(\mathbf{v}_{2}^{T} \mathbf{v}_{1}\right) \mathbf{v}_{1}\right)$ in equation (5.39) have the general form of $\left(\mathbf{b}_{j}-\left(\mathbf{b}_{j}^{T} \mathbf{b}_{i}\right) \mathbf{b}_{i}\right)$. The covariance of this vector, $\Omega\left(\mathbf{b}_{j}-\left(\mathbf{b}_{j}^{T} \mathbf{b}_{i}\right) \mathbf{b}_{i}\right)$ is:

$$
\begin{aligned}
\Omega\left(\mathbf{b}_{j}-\left(\mathbf{b}_{j}^{T} \mathbf{b}_{i}\right) \mathbf{b}_{i}\right) & =\Omega\left(v_{j}\right)+\mathbf{b}_{j}^{T} \mathbf{b}_{i} \Omega\left(v_{i}\right)-\mathbf{b}_{j} \mathbf{b}_{j}^{T} \odot\left[C\left(\mathbf{b}_{i}\right)-\Omega\left(v_{i}\right)\right]-\mathbf{b}_{i} \mathbf{b}_{i}^{T} \odot\left[C\left(\mathbf{b}_{j}\right)-\Omega\left(v_{j}\right)\right] \\
& +\mathbf{b}_{i} \mathbf{b}_{i}^{T}\left[\left(\mathbf{b}_{j} \odot \mathbf{b}_{j}\right)^{T} \sigma_{\mathbf{b}_{i}}^{2}+\left(\mathbf{b}_{i} \odot \mathbf{b}_{i}\right)^{T} \sigma_{\mathbf{b}_{j}}^{2}\right]+2 \mathbf{b}_{j}^{T} \mathbf{b}_{i}\left[\left(\mathbf{b}_{i} \mathbf{b}_{j}^{T}+\mathbf{b}_{j} \mathbf{b}_{i}^{T}\right) \odot M\left(\sigma_{\mathbf{b}_{i}}^{2}\right)\right]
\end{aligned}
$$

where,

$$
C\left(\mathbf{b}_{i}\right)=M\left(\sigma_{\mathbf{b}_{i}}^{2}\right)+M^{T}\left(\sigma_{\mathbf{b}_{i}}^{2}\right)
$$

The elements of the vector, $\sigma_{\mathbf{b}_{i}}^{2}$ are the diagonal elements of the measurement covariance matrix $\Omega\left(v_{i}\right)$. 
In a similar manner, $R_{4}$ can be calculated as:

$$
\begin{aligned}
R_{4} & =E\left\{\left(\mathbf{w}_{4}-A_{1}^{2} \mathbf{v}_{4}\right)\left(\mathbf{w}_{4}-A_{1}^{2} \mathbf{v}_{4}\right)^{T}\right\} \\
& =\left(\frac{\partial \mathbf{w}_{4}}{\partial \mathbf{r}_{3}}\right) \Omega\left(\mathbf{w}_{1} \times \mathbf{s}\right)\left(\frac{\partial \mathbf{w}_{4}}{\partial \mathbf{r}_{3}}\right)^{T}+A_{1}^{2}\left(\frac{\partial \mathbf{v}_{4}}{\partial \mathbf{r}_{4}}\right) \Omega\left(\mathbf{v}_{1} \times \mathbf{v}_{2}\right)\left(\frac{\partial \mathbf{v}_{4}}{\partial \mathbf{r}_{4}}\right)^{T} A_{1}^{2^{T}}
\end{aligned}
$$

where the $\mathbf{r}_{3}$ represents $\left(\mathbf{w}_{1} \times \mathbf{s}\right), \mathbf{r}_{4}$ represents $\left(\mathbf{v}_{1} \times \mathbf{v}_{2}\right)$. The covariance between the two cross product vectors, $\mathbf{b}_{i} \times \mathbf{b}_{j}, \Omega\left(\mathbf{b}_{i} \times \mathbf{b}_{j}\right)$ is:

$$
\Omega\left(\mathbf{b}_{i} \times \mathbf{b}_{j}\right)=S\left(\mathbf{b}_{i}\right) S\left(\mathbf{b}_{i}\right)^{T}+S\left(\mathbf{b}_{j}\right) S\left(\mathbf{b}_{j}\right)^{T}
$$

with,

$$
\begin{gathered}
S\left(\mathbf{b}_{i}\right)=\left[\mathbf{b}_{i} \times\right] \odot M\left(\sigma_{\mathbf{b}_{i}}\right) \\
\sigma_{\mathbf{b}_{i}}=\left[\begin{array}{lll}
\left(\sigma_{x}\right)_{\mathbf{b}_{i}} & \left(\sigma_{y}\right)_{\mathbf{b}_{i}} & \left(\sigma_{z}\right)_{\mathbf{b}_{i}}
\end{array}\right]^{T}
\end{gathered}
$$

Both $R_{2}$ and $R_{3}$, derived in equations (5.39) and (5.42), are singular in this case. However, Shuster has shown that this type of matrices can be modified to become nonsingular matrices [113]. For example, the matrix $R_{2}$ may be replaced by $R_{2}^{\text {new }}=R_{2}+\frac{1}{2} \mathbf{b b}^{T} \operatorname{trace}\left(R_{2}\right)$, where $\mathbf{b}=\left(\mathbf{s}-\mathbf{s}^{T} \mathbf{w} \mathbf{w}\right) /\left(\left\|\mathbf{s}-\mathbf{s}^{T} \mathbf{w} \mathbf{w}\right\|\right)$. Then, both $R_{2}^{\text {new }}$ and $R_{3}^{\text {new }}$ are invertible.

\subsection{Relative Attitude Determination - Non-Parallel Case}

Figure 5.3 shows the four LOS measurements in three-spacecraft formation for the non-parallel case. The vectors, $\mathbf{v}_{1}, \mathbf{v}_{2}, \mathbf{w}$ and $\mathbf{s}$ have been defined in the Section 5.2. Here, figure 5.3 show that the vectors $\mathbf{v}_{1}$ and $\mathbf{v}_{2}$ become out of the plane formed by the vectors $\mathbf{w}$ and $\mathbf{s}$. Thus, this results in a non-parallel LOS measurement case.

Let $F_{1}$ be the frame formed by the vector $\mathbf{v}_{1}$ and $\mathbf{v}_{2}$, and $F_{2}$ be the plane formed by the vector $\mathbf{w}$ and $\mathbf{s}$, which can be defined as:

$$
\begin{gathered}
F_{1} \rightarrow \mathbf{v}_{2} \times \mathbf{v}_{1} \\
F_{2} \rightarrow \mathbf{w} \times \mathbf{s}
\end{gathered}
$$




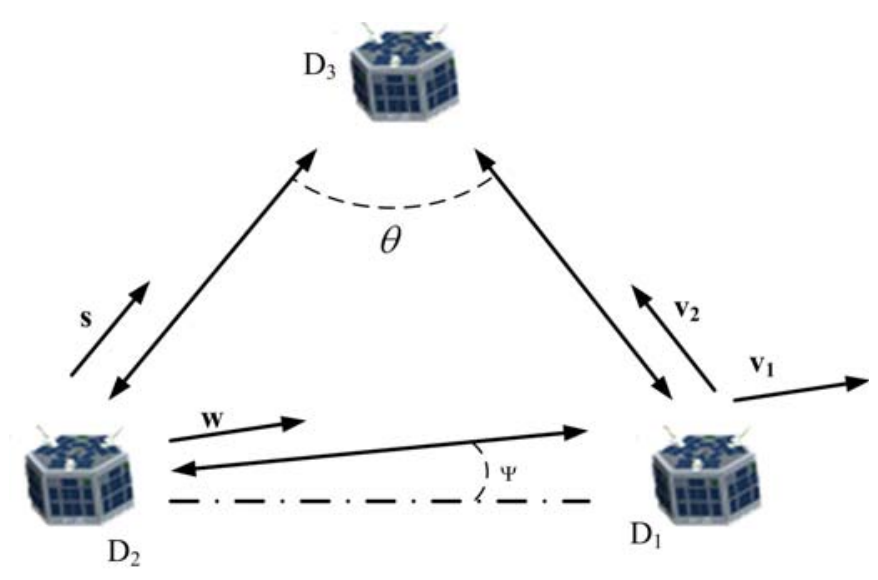

Figure 5.3: The line-of-sight measurement in three-spacecraft formation for non-parallel case.

Note that $\mathbf{v}_{1}$ and $\mathbf{v}_{2}$ are expressed in terms of $D_{1}$ spacecraft reference frame, and $\mathbf{s}$ and $\mathbf{w}$ are expressed in terms of $D_{2}$ spacecraft reference frame. Therefore, we can define two relative attitude matrices, $A_{1}^{F_{1}}$ and $A_{2}^{F_{2}}$, which are the relative attitude between $D_{1}$ spacecraft reference frame and $F_{1}$ frame, and the relative attitude between $D_{2}$ spacecraft reference frame and $F_{2}$ frame using the similar method developed in Section 5.2. $A_{1}^{F_{1}}$ and $A_{2}^{F_{2}}$ are given as:

$$
\begin{gathered}
A_{1}^{F_{1}}=\left[\begin{array}{lll}
\mathbf{v}_{1} & \frac{\mathbf{v}_{2} \times \mathbf{v}_{1}}{\left\|\mathbf{v}_{2} \times \mathbf{v}_{1}\right\|} & \frac{\mathbf{v}_{2}-\left(\mathbf{v}_{2}^{T} \mathbf{v}_{1}\right) \mathbf{v}_{1}}{\left\|\mathbf{v}_{2}-\left(\mathbf{v}_{2}^{T} \mathbf{v}_{1}\right) \mathbf{v}_{1}\right\|}
\end{array}\right] \\
A_{2}^{F_{2}}=\left[\begin{array}{lll}
\mathbf{w} & \frac{\mathbf{s} \times \mathbf{w}}{\|\mathbf{s} \times \mathbf{w}\|} & \frac{\mathbf{s}-\left(\mathbf{s}^{T} \mathbf{w}\right) \mathbf{w}}{\left\|\mathbf{s}-\left(\mathbf{s}^{T} \mathbf{w}\right) \mathbf{w}\right\|}
\end{array}\right]
\end{gathered}
$$

First, we consider the angle between vector $\mathbf{v}_{2}$ and $\mathbf{s}$ as one of the cost function in order to determine the relative attitude matrix between any two spacecraft in the formation:

$$
\mathbf{v}_{2}^{T} A_{2}^{1} \mathbf{s}=\cos \theta
$$

Equation (5.51) can be also expressed in terms of $A_{1}^{F_{1}}$ and $A_{2}^{F_{2}}$, which corresponds to:

$$
\begin{aligned}
\cos \theta & =\left(A_{1}^{F_{1}} \mathbf{v}_{2}\right)^{T} A_{F_{2}}^{F_{1}} A_{2}^{F_{2}} A_{2}^{1} \mathbf{s} \\
& =\mathbf{e}_{1}^{T} A_{F_{2}}^{F_{1}} \mathbf{e}_{2}
\end{aligned}
$$


where,

$$
\begin{gathered}
\mathbf{e}_{1}=A_{1}^{F_{1}} \mathbf{v}_{2} \\
\mathbf{e}_{2}=A_{2}^{F_{2}} A_{2}^{1} \mathbf{s}
\end{gathered}
$$

The attitude matrix, $A_{F_{2}}^{F_{1}}$, between $F_{1}$ and $F_{2}$ frame can be expressed in terms of principal angle, $\Psi$ [74], which is also the out-of-plane angle in Figure 5.3. The $A_{F_{2}}^{F_{1}}$ is given as:

$$
A_{F_{2}}^{F_{1}}=\cos \Psi I_{3 \times 3}+(1-\cos \Psi) \bar{n} \bar{n}^{T}-\sin \Psi[\bar{n} \times]
$$

where $I_{3 \times 3}$ is 3 by 3 identity matrix and $\bar{n}=A_{2}^{F_{2}} \mathbf{w}$.

Because $\mathbf{w}$ is the first column vector in $A_{2}^{F_{2}}$, thus, it can be shown that,

$$
\bar{n}=\left[\begin{array}{lll}
1 & 0 & 0
\end{array}\right]^{T}
$$

We define the elements of both vectors, $\mathbf{e}_{1}$ and $\mathbf{e}_{2}$, as $\mathbf{e}_{1}=\left[\begin{array}{lll}e_{11} & e_{12} & e_{13}\end{array}\right]^{T}$ and $\mathbf{e}_{2}=\left[\begin{array}{lll}e_{21} & e_{22} & e_{23}\end{array}\right]^{T}$ respectively. Then, by substituting equations (5.54) and (5.55) into equation (5.51), we obtain:

$$
\left(e_{12} e_{22}+e_{13} e_{23}\right) \cos \Psi+\left(e_{13} e_{22}+e_{12} e_{23}\right) \sin \Psi=\cos \theta-e_{11} e_{21}
$$

Note that both $\mathbf{v}$ and $\mathbf{s}$ are perpendicular to one of the column vectors in $\left(A_{1}^{F_{1}}\right.$ and $\left(A_{2}^{F_{2}}\right.$ respectively. Therefore, either the second or the third element in each $\mathbf{e}_{1}$ and $\mathbf{e}_{2}$ vector is always equal to zero, which implies that the term $e_{13} e_{22}+e_{12} e_{23}=0$. Then, equation is simplified to:

$$
\left(e_{12} e_{22}+e_{13} e_{23}\right) \cos \Psi=\cos \theta-e_{11} e_{21}
$$

Finally, a closed form solution for $\Psi$ can be obtained:

$$
\Psi=\cos ^{-1}\left(\frac{\cos \theta-e_{11} e_{21}}{e_{12} e_{22}+e_{13} e_{23}}\right)
$$

Equation (5.58) states that there are always two principal angles, $\Psi$, that can be obtained. Therefore, there are always two possible solution for the relative attitude between two 
spacecraft. Then, the relative attitude matrix between $D_{1}$ and $D_{2}$ can be calculated as:

$$
A_{1}^{2}=A_{1}^{F_{1}} A_{F_{1}}^{F_{2}}\left(A_{2}^{F_{2}}\right)^{T}
$$

\subsubsection{Covariance Analysis - Non-Parallel Case}

Here, the covariance of relative attitude determination error between any two spacecraft in the formation is derived. We consider equation (5.50) as one of the cost function and the relationship between $\mathbf{v}_{1}$ and $\mathbf{w}$ given in equation (5.17) as the other cost function. Then, we can construct the error function for the non-parallel case as follows:

$$
\begin{aligned}
J & =\frac{1}{2}\left\{\left(\mathbf{p}_{31}^{3}\right)^{T} \mathbf{p}_{32}^{3}-\left(\mathbf{p}_{23}^{2}\right)^{T} A_{1}^{2} \mathbf{p}_{13}^{1}\right\}^{2} R_{\theta}^{-1} \\
& +\frac{1}{2}\left\{\mathbf{p}_{21}^{2}-A_{1}^{2} \mathbf{p}_{21}^{1}\right\}^{T}\left(R_{21}^{2}\right)^{-1}\left\{\mathbf{p}_{21}^{2}-A_{1}^{2} \mathbf{p}_{21}^{1}\right\}
\end{aligned}
$$

A similar covariance analysis method, as shown in Section 5.2.1, is applied on equation (5.60). Then, the corresponding covariance is given as [12]:

$$
\begin{aligned}
P_{1}^{2} & =\left(\left[A_{1}^{2} \mathbf{p}_{31}^{1} \times\right] \mathbf{p}_{32}^{2} R_{\theta}^{-1}\left(\mathbf{p}_{32}^{2}\right)^{T}\left[A_{1}^{2} \mathbf{p}_{31}^{1} \times\right]^{T}+\left[A_{1}^{2} \mathbf{p}_{21}^{1} \times\right]\left(R_{21}^{2}\right)^{-1}\left[A_{1}^{2} \mathbf{p}_{21}^{1} \times\right]^{T}\right)^{-1} \\
& =\left(\left[\mathbf{p}_{31}^{2} \times\right] \mathbf{p}_{32}^{2} R_{\theta}^{-1}\left(\mathbf{p}_{32}^{2}\right)^{T}\left[\mathbf{p}_{31}^{2} \times\right]^{T}+\left[\mathbf{p}_{21}^{2} \times\right]\left(R_{21}^{2}\right)^{-1}\left[\mathbf{p}_{21}^{2} \times\right]^{T}\right)^{-1}
\end{aligned}
$$

As discussed in Section 5.2.1, the Fisher Information Matrix of equation (5.61) is required to be non-singular at anytime. Let the Fisher Information Matrix is:

$$
F=\left[\mathbf{p}_{31}^{2} \times\right] \mathbf{p}_{32}^{2} R_{\theta}^{-1}\left(\mathbf{p}_{32}^{2}\right)^{T}\left[\mathbf{p}_{31}^{2} \times\right]^{T}+\left[\mathbf{p}_{21}^{2} \times\right]\left(R_{21}^{2}\right)^{-1}\left[\mathbf{p}_{21}^{2} \times\right]^{T}
$$

The equation would only become singular if and only if $\mathbf{p}_{21}^{2}$ falls in the same plane as $\mathbf{p}_{31}^{2}$ and $\mathbf{p}_{32}^{2}$. Therefore, the Fisher Information Matrix in equation (5.62) has no singularity issue except the $\Psi$ angle (see Figure 5.3) is close to zero. One method that can be taken to avoid the singularity is to check the $\phi$ angle. If $\phi$ is small enough, e.g. $\phi$ less than 0.1 degrees, then the covariance in equation (5.31) for the parallel case is considered. 


\subsubsection{Measurement covariance - Non-Parallel Case}

Here, the measurement covariance which corresponds to the covariance matrix in (5.61) is presented. Both $R_{21}^{2}$ and $R_{\theta}$ are required to be calculated in order to determine the covariance in equation (5.61). The $R_{21}^{2}$ can be calculated in a straight forward manner as shown in equation (5.35), which is given as:

$$
R_{21}^{2}=\Omega_{21}^{2}+A_{1}^{2} \Omega_{21}^{1}\left(A_{1}^{2}\right)^{T}
$$

where $\Omega_{21}^{2}$ denotes the measurement noise covariance for the measurement $\mathbf{p}_{21}^{2}$, and $\Omega_{21}^{1}$ denotes the measurement noise covariance for the measurement $\mathbf{p}_{21}^{1}$. In addition, $\Omega$ is defined in equation (5.34).

The $R_{\theta}$ in equation (5.60) has been derived in Ref. [12], which is given as:

$$
R_{\theta}=2\left\{\left(\mathbf{p}_{32}^{3}\right)^{T} \Omega_{31}^{3} \mathbf{p}_{32}^{3}+\left(\mathbf{p}_{31}^{3}\right)^{T} \Omega_{32}^{3} \mathbf{p}_{31}^{3}+\operatorname{Tr}\left(\Omega_{31}^{3} \Omega_{32}^{3}\right)\right\}
$$

where $\operatorname{Tr}($.$) denotes the sum of the diagonal elements of the matrix, and both \Omega_{31}^{3}$ and $\Omega_{31}^{3}$ are calculated in the similar method as in equation (5.63).

In Section 5.2.1, the measurement covariance calculated experiences a singularity issue. However, the singularity issue can be avoided by calculating a new covariance, $R^{\text {new }}=R+\frac{1}{2} \mathbf{b b}^{T} \operatorname{Tr}(R)$. Here, $R_{21}^{2}$ is always non-singular. In addition, $R_{\theta}$ is a scalar function, which guarantees the non-singularity except $R_{\theta}=0$.

\subsection{Simulation}

We consider an isosceles triangle shape of three spacecraft formation, such that $\theta_{1}=\theta_{2}$, see Figure 5.4. Each spacecraft has its absolute attitude expressed in the Earth Center Inertial (ECI) frame. We describe the spacecraft attitude using the 3-1-3 rotation sequence $[22,74]$. The orientation of $D_{1}$ is $A_{1}\left(30^{\circ}, 15^{\circ},-20^{\circ}\right), D_{2}$ is $A_{2}\left(25^{\circ}, 15^{\circ}, 30^{\circ}\right)$, and $D_{3}$ is 


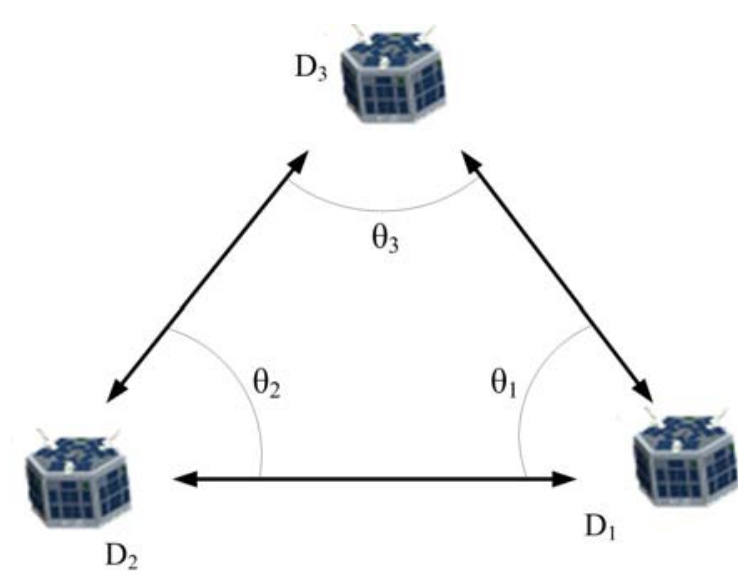

Figure 5.4: Three Spacecraft Formation Configuration.

$A_{3}\left(30^{\circ}, 0^{\circ}, 10^{\circ}\right)$. The attitude matrix is:

$$
A_{i}\left(\phi_{1}, \phi_{2}, \phi_{3}\right)=\left[\begin{array}{ccc}
c \phi_{3} c \phi_{1}-s \phi_{3} c \phi_{2} s \phi_{1} & c \phi_{3} s \phi_{1}+s \phi_{3} c \phi_{2} c \phi_{1} & s \phi_{3} s \phi_{2} \\
-s \phi_{3} c \phi_{1}-c \phi_{3} c \phi_{2} s \phi_{1} & -s \phi_{3} s \phi_{1}+c \phi_{3} c \phi_{2} c \phi_{1} & c \phi_{3} s \phi_{2} \\
s \phi_{2} s \phi_{1} & -s \phi_{2} c \phi_{1} & c \phi_{2}
\end{array}\right]
$$

where $c$ denotes cosine and $s$ denotes sine.

The orientation of all spacecraft in the formation are the same in both the parallel and non-parallel case. However, the line-of-sight measurement between each spacecraft is varied for both cases.

\subsubsection{Parallel Case}

For simplicity, we assume that the plane of the formation is parallel to the Equatorial plane, and the observation vector of $D_{1}$ spacecraft measured by $D_{2}$ spacecraft, expressed in ECI frame, is:

$$
\mathbf{p}_{21}^{I}=\left[\begin{array}{lll}
1 & 0 & 0
\end{array}\right]^{T}
$$

$I$ denotes the ECI frame of reference, and the observation vector of $D_{3}$ spacecraft measured by $D_{1}$ spacecraft, expressed in ECI frame, is:

$$
\mathbf{p}_{13}^{I}=\left[\begin{array}{lll}
-\cos \theta_{1} & \sin \theta_{1} & 0
\end{array}\right]^{T}
$$



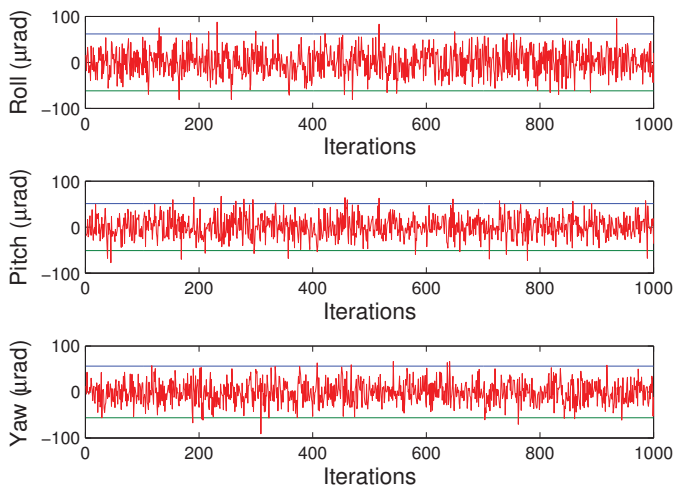

Figure 5.5: Errors in the relative attitude $A_{d_{1}}^{d_{2}}$.
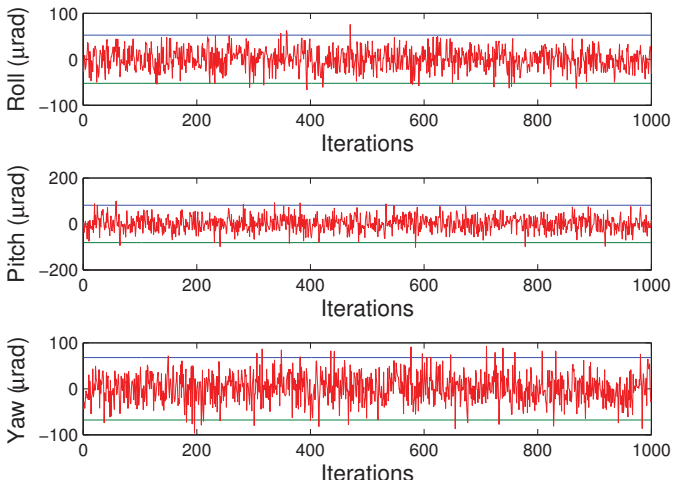

Figure 5.6: Errors in the relative attitude $A_{d_{3}}^{d_{1}}$.

and the observation vector of $D_{3}$ spacecraft measured by $D_{2}$ spacecraft, expressed in ECI frame, is:

$$
\mathbf{p}_{23}^{I}=\left[\begin{array}{lll}
\cos \theta_{2} & \sin \theta_{2} & 0
\end{array}\right]^{T}
$$

The measurement vector, $\mathbf{p}_{i j}$ expressed in $k^{t h}$ spacecraft reference frame is given by $\mathbf{p}_{i j}^{k}=A_{k} \mathbf{p}_{i j}^{I}$.

For the simulation, all relative position measurements are assumed to be unit vectors. The measurement noise is assumed to be a linear additive, with the standard deviation of $17 \times 10^{-6}$ radian on each axis. In order to study the effect of the shape of the spacecraft formation on the relative attitude determination error, we vary the value of $\theta_{1}$ from 1 to 89 degrees in increments of 1 degree. The angle $\theta_{3}$ can be obtained since $\theta_{2}=\theta_{1}$. For each increment of $\theta_{1}$, the simulation is run for 1000 iterations. The average of Root Mean Square Error (RMSE) over the 1000 iterations is plotted to study the performance in the simulation.

Next, the performance of the RAD method is studied for a range of levels of the measurement noise. The equilateral formation is considered. The measurement noise is varied from $1 \mu \mathrm{rad}$ to $100 \mu \mathrm{rad}$ in increments of $1 \mu \mathrm{rad}$. The simulation is run over 1000 iterations for each level of measurement noise. The mean of absolute error is compared with the analytical error, which is the three sigma boundary of the covariance.

Figures 5.5, 5.6, and 5.7 show the three sigma boundary of relative attitude error in roll, pitch, and yaw angles, respectively. The relative attitude error vector is computed using 

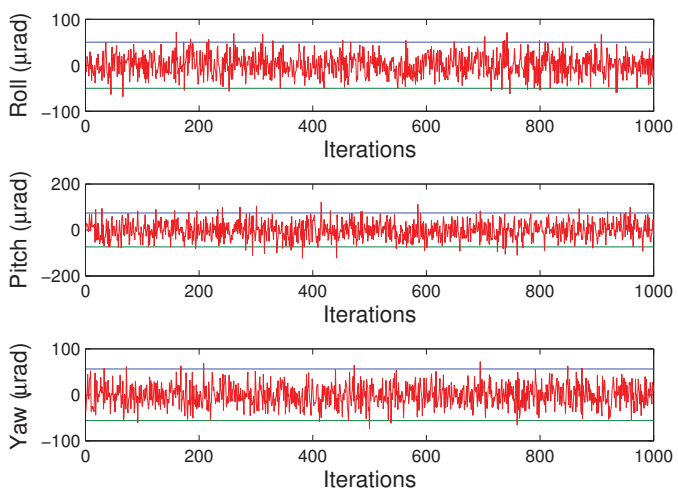

Figure 5.7: Errors in the relative attitude $A_{d_{3}}^{d_{2}}$.

equation (5.28). The figures show that for 1000 iterations, the relative attitude determination errors fall within the three sigma boundary, which means that the relative attitude between spacecraft can be determined, using equations (5.5) to (5.12), with a known expected accuracy level. The measurements covariances derived in equations (5.39) and (5.42) are singular in nature. However, these matrices were replaced by other nonsingular matrices as discussed in section 5.2.2.

Figure 5.8 shows the change of RMSE of relative attitude determination between the $D_{2}$ and $D_{3}$ spacecraft, as the shape of spacecraft formation changes. When $\theta_{1}$ is very small (that is the $D_{3}$ spacecraft is almost colinear with the $D_{1}$ and $D_{2}$ spacecraft), the RMSE is higher. The RMSE is also high when $\theta_{1}$ becomes very large $\left(D_{1}\right.$ and $D_{2}$ spacecraft

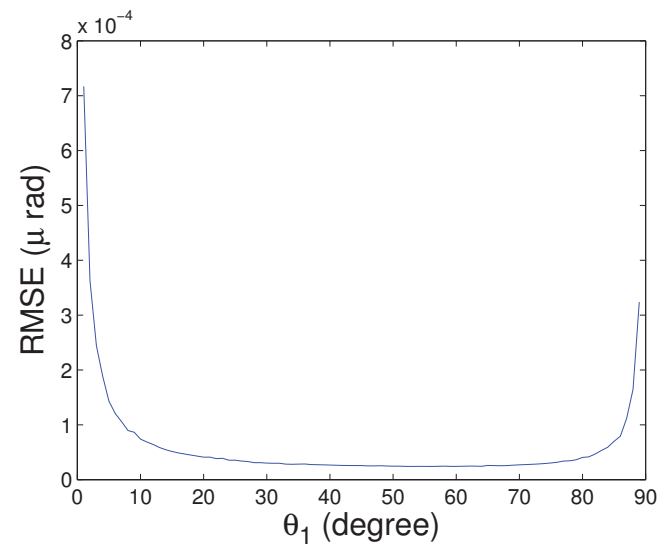

Figure 5.8: Th RMSE of $A_{d_{c}}^{d_{2}}$ with respect to change of $\theta_{1}$. 


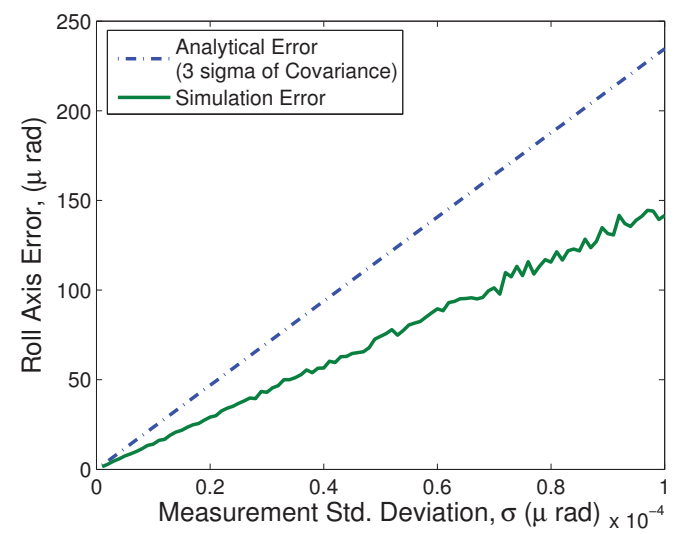

Figure 5.9: Comparison between Analytical and Simulation Error with respect to measurement noise standard deviation.

become very close to each other). In both cases, the shape of the formation becomes close to a straight line (colinear). Other than the previous two special cases, the proposed method is capable of providing accurate results with errors below $100 \mu \mathrm{rad}$.

Figure 5.9 compares the analytical and simulation error as the measurements noises vary. The measurements noises in the figure are expressed in terms of standard deviation. The analytical error is obtained through the determination of the three sigma boundary using the covariance of relative attitude between two spacecraft (derived in equation (5.31)). As expected, both analytical and simulation errors increase as the measurements noises increase. In Figure 5.9, only the error on the roll axis of relative attitude between spacecraft 2 and spacecraft 3 is considered. The errors on the pitch and yaw axes have the same characteristics as the error on the roll axis. The three sigma boundary represents the boundary that the RAD error would fall within. The average simulated error for each increment is lower than the analytical error.

In some formation scenarios, the accuracy of estimated absolute attitude is in the order of $1 \times 10^{-5}$ degrees of error on each axis $[114,115]$. The relative attitude errors, as estimated from absolute attitudes, are determined using the quaternion multiplication properties: $\hat{\mathbf{q}}=\mathbf{q} \otimes \delta \mathbf{q}$, and $\mathbf{q}_{12}=\mathbf{q} \otimes \mathbf{q}_{1}^{-1}$, where $\hat{\mathbf{q}}$ is the estimated quaternion, $\mathbf{q}$ is the true quaternion, $\mathbf{q}_{12}$ is the relative quaternion between spacecraft $D_{1}$ and $D_{2}$, and $\delta \mathbf{q}$ is the attitude error quaternion $[22,114]$.

The accuracy of the proposed RAD method is compared to the accuracy of the RAD presented in Ref. [12], and the relative attitude determined using the absolute attitude. 
Figures 5.5 to 5.7 show that the accuracy of the proposed RAD method falls within $100 \mu \mathrm{rad}$ (or about 0.005 degrees). The relative attitude errors presented in Ref. [12] fall within $100 \mu \mathrm{rad}$. Both RAD methods process equivalent accuracy. But the RAD method presented in this chapter is not limited by any constraint as in Ref. [12]. If the accuracy of absolute attitude is $1 \times 10^{-5}$ degrees of error, the relative attitude errors will be in the order of $1 \times 10^{-4}$ degrees, on each axis. However, each spacecraft is required to be equipped with a full set of star trackers to achieve the particular accuracy [116]. In terms of algorithm complexity, the proposed RAD method requires 15 multiplication steps to calculate the relative attitude matrix. In most cases, the absolute attitude is expressed in terms of the quaternion vectors. This requires 12 multiplication steps to calculate the relative quaternion vectors, and another 12 multiplication steps to calculate the relative attitude using the relative quaternion vector.

\subsubsection{Non-Parallel Case}

Next, the relative attitude method developed for the non-parallel case is studied. Three LOS measurements, $\mathbf{p}_{31}^{3}, \mathbf{p}_{32}^{3}$, and $\mathbf{p}_{12}^{1}$ are given as:

$$
\begin{gathered}
\mathbf{p}_{31}^{3}=\left[\begin{array}{lll}
\sin \left(-30^{\circ}\right) \cos \left(35^{\circ}\right) & \sin \left(35^{\circ}\right) & \cos \left(30^{\circ}\right) \cos \left(35^{\circ}\right)
\end{array}\right]^{T} \\
\mathbf{p}_{32}^{3}=\left[\begin{array}{lll}
\sin \left(30^{\circ}\right) \cos \left(25^{\circ}\right) & \sin \left(25^{\circ}\right) & \cos \left(30^{\circ}\right) \cos \left(25^{\circ}\right)
\end{array}\right]^{T} \\
\mathbf{p}_{12}^{1}=\left[\begin{array}{lll}
-\cos \left(45^{\circ}\right) \cos \left(10^{\circ}\right) & \sin \left(10^{\circ}\right) & \sin \left(-45^{\circ}\right) \cos \left(10^{\circ}\right)
\end{array}\right]^{T}
\end{gathered}
$$

If the true relative attitude is known, all six LOS measurements can be obtained from equations (5.69) to (5.71). Similar to the previous case study, a simulation is set up for 1000 iterations run. In addition, the measurement noise standard deviation for each axis of the LOS measurement is $\sigma=17 \times 10^{-6}$ radian.

Figure 5.10 presents the two relative attitude error in three sigma boundary. Recall from equation (5.58) that there are two possible $\Psi$ solutions. Here, the corresponding principal angles, $\Psi$, calculated are 12.54 degrees and 347.46 degrees respectively. Althought both solutions are able to show that $\mathbf{p}_{21}^{2}=A_{1}^{2} \mathbf{p}_{21}^{1}$, only one of the $\Psi$ solutions presents the actual relative attitude between the two spacecraft ( $D_{1}$ and $D_{2}$ spacecraft). Figure 5.10(a) shows that the relative attitude obtained using $\Psi=12.54$ degrees does not match the true 

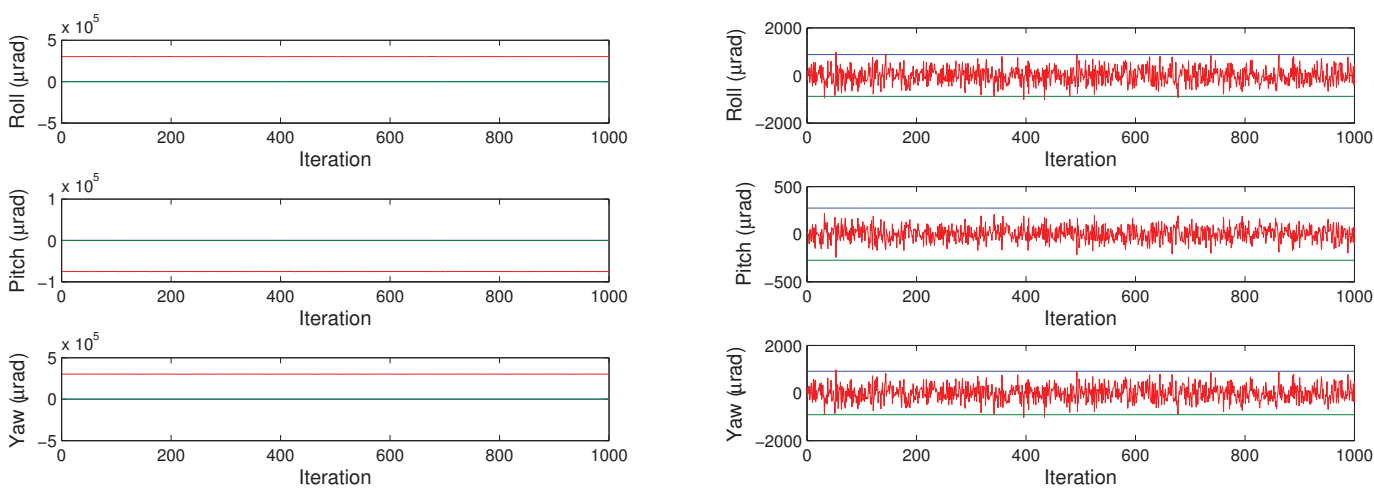

(a) First Psi solution.

(b) Second Psi solution.

Figure 5.10: Relative attitude error of $A_{1}^{2}$ in three sigma boundary for two Psi solution.

relative attitude. This results in relative attitude errors always out of the three sigma bound. However, the other relative attitude obtained using $\Psi=347.46$ does match the true relative attitude, which is shown in Figure 5.10(b). Furthermore, the result in Figure 5.10(b) also shows that the error always falls within the three sigma boundary. 


\section{Chapter 6}

\section{A Weighted Measurement Fusion Kalman Filter Implementation for UAV Navigation}




\subsection{Introduction}

Vehicular navigation has a variety of civilian and military applications and has been widely studied since the last century. The radar sensor has been widely used in vehicle tracking applications [75]. Recent technology advancement enabled the development of small-size antenna arrays, such as the Wireless Local Positioning System (WLPS) for measuring position[58]. Navigation systems based on the Global Positioning System (GPS) can precisely estimate position; yet the GPS signal can be jammed. Several methods are proposed in the literature to aid vehicle navigation in GPS-denied environments [117]. Examples of these methods include the Inertial Navigation System (INS) and the Terrain Aided Navigation System (TANS). The INS uses motion sensors to calculate the position of a vehicle with respect to a bearing point, and the TANS uses the terrain's image stored in the database to estimate the vehicle position. The INS navigation errors, however, may become high if the propagation errors are high [118]. Also, the TANS may not perform well in unknown terrain environments [119].

The Kalman Filter (KF) has been widely used in many navigation applications. Ref. [119] implements the KF in estimating moving a vehicle's position and orientation, using GPS, INS, and TANS. One way to process measurements in a multisensor system is to combine all the measurements into a single observation vector in the KF. The computational load of this implementation, however, increases as the number of measurements increases [35]. Ref. [36] presents a State-Vector Fusion (SVF) method in which each measurement is processed by its own local filter simultaneously. Then, the updated estimated states and the predicted covariances are fused together [37]. The Measurement Fusion (MF) method, introduced in Ref. [38], fuses multiple measurements and then the KF is applied to the result. Ref. [39] has analytically shown that, under certain conditions, the MF method is similar to the standard KF. Both SVF and MF methods require less computational load compared to the standard KF [38]. However, the derivation of these fusion methods assumes uncorrelated measurement noise for multisensor systems [37, 40]. In most of the multisensor systems, the sensors' noises are correlated due to the interference signal between sensors [40]. Ref. [41] has presented a weighted MF method in which the input and measurement noises could be correlated. In addition, the computational load of the weighted MF method is significantly lower than the KF [41].

Time measurements errors due to signal travel time and processing time (also known as 
out-of-sequence measurement (OOSM) problem) have been studied in the literature $[69,120]$. Several estimation algorithms that account for these errors have been proposed [64, 120]. Ref. [84] implements the Unscented Kalman Filter (UKF) to estimate these errors, and demonstrates a relatively high estimation accuracy. However, it has a higher computational cost [84]. Ref. [121] implements a Particle Filter to solve the same problem. Particle Filter implementation provides a suboptimal estimation and its computational cost is high [122].

In this paper, it is assumed that UAVs are equipped with a Wireless Local Positioning System (WLPS) at all times [58]. The WLPS enables UAVs to find their own position with respect to some bearing points with known positions, via Time-of-Arrival (TOA) and Direction-of-Arrival (DOA) measurements. The WLPS consists of two components, a Dynamic Base Station (DBS) and a transponder (TRX). The DBS installed on the UAV communicates with all the TRXs installed at known positions (e.g. on the ground or on another vehicle such as the Airborne Warning and Control System (AWACS) aircrafts) and localizes them via DOA and TOA measurements. Identification codes are allocated to each TRX such that DBSs can identify different TRXs [58].

Given that the position of a TRX is known at all times, with a given accuracy, the position and velocity of the UAV will be estimated. A Weighted Measurement Fusion Kalman Filter (WMFKF), based on the MF method [39], will be implemented for estimation. In the proposed WMFKF, all measurements from all TRXs are fused into one. Because the measurements are received at different times at the DBS, a weighting factor is computed for each measurement; this weighting factor is proportional to the time difference between the measurement time and the time of fusing all the measurements. The resulting fused measurement and its corresponding noise covariance are then processed in a way similar to a KF. This weighting factor reduces the convergence rate of the predicted covariance to ensure that estimation errors are within the three sigma boundary. The accuracy performance of the WMFKF is compared to the standard KF. The error distribution probability, also known as the Ellipsoid Error Probable (EEP), for the fused measurements in the WMFKF method is evaluated and compared to the fused measurement in the MF Kalman Filter (MFKF) presented in Ref. [38]. Also, the computational complexity of the WMFKF is analyzed and compared to that of the standard KF. Two scenarios are considered in this paper: (1) GPS-denied environment, and (2) available GPS measurements. 
The purpose of this chapter is to explore use of TOA as a weighting factor in the fusion process to improve the estimation stability. This implementation results in maintaining the estimated state error within the estimated covariance boundaries, as shown in the results presented. This implementation also avoids the complexity of estimating the measurements' time delays. In this chapter, the number of available TRXs varies over time due to limited wireless transmission range. Also, the DBS transmits a signal after all response signals from all TRXs are received (they arrive at different times due to time delay errors.) The position of the TRXs are not perfectly known. This paper is organized as follows: Section 6.2 presents the formulation of the navigation problem. Section 6.3 presents the WMFKF method. Section 6.4 presents how the EEP is computed. Sections 6.5 presents the simulation results.

\subsection{Problem Formulation}

A moving vehicle is assumed to travel from an origin to a destination and one or more TRX(s) are placed along the vehicle's travel path to assist the navigation system. The vehicle is assumed to be equipped with only the DBS. It should be noted that the main complexity of the WLPS is in the DBS, as it needs to be equipped with antenna arrays to allow complex signal processing schemes for DOA-TOA estimation, beamforming, localization, and tracking. However, the complexity of the TRX is very low when it is compared to the DBS. The TRX is composed of a single antenna transmitter and receiver. The role of the TRX is to detect the DBS's signal and send a signal back to the DBS. Therefore, multiple TRXs can be easily distributed in or around the path from origin to destination. In Fig. 6.1, the DBS transmits periodic signals called ID request (IDR) to the TRXs that fall within its coverage area. TRX responds back to the DBS as soon as it receives the IDR signal. The DBS measures the TOA by calculating the total time required by the signal to be transmitted from the DBS to the TRX, and back. This two-way TOA measurement method avoids the need for clock synchronization. A two-way TOA measurement, however, experiences signal transmission delay, multipath effects, and higher atmospheric delay, which might reduce the accuracy of the measurement.

The DBS receives the response signal from each TRX at a different time; this is because the distances between the TRXs and the DBS are different. In Fig. 6.1, TRX 1 is closer to the DBS compared to TRX 2. Thus, $t_{1}$, the TRX 1 signal arrival time is less than $t_{2}$, the 


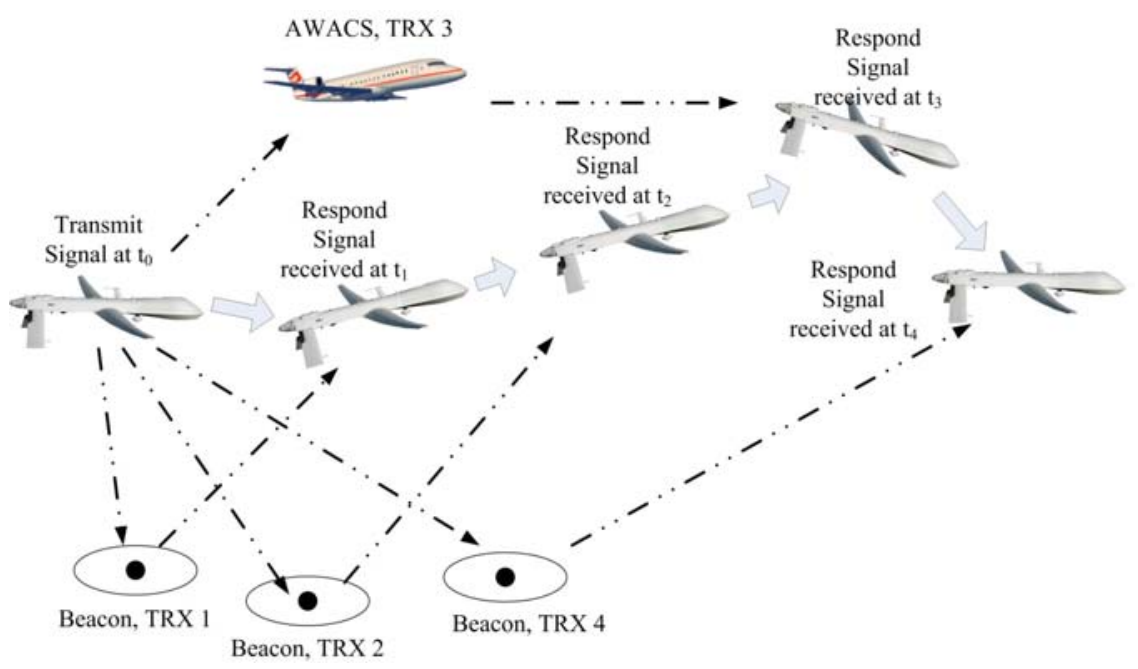

Figure 6.1: Illustration of transmission between DBS and TRX.

TRX 2 signal arrival time. Considering $N$ number of TRXs, the DBS receives the response signals from the TRXs at the times $t_{1}, t_{2} \ldots, t_{N}$. The updating step is applied after all TRXs' measurements are received at the DBS, i.e., at $t=t_{N}$ (see Fig. 6.2). The position errors due to these time delays in the measurements processing are not computed, but rather these delays are accounted for through the weighting factor, as detailed in Section 6.3

Let the position of the DBS at time $t_{i}$ be $\mathbf{r}_{t_{i}}$, and the position for the $i^{t h}$ TRX be $\mathbf{r}_{B, i}$, respectively. The TOA measurement between the DBS and the $i^{\text {th }}$ TRX is:

$$
\tilde{y}_{i, T}=\left\|\mathbf{r}_{t_{0}}-\mathbf{r}_{B, i}\right\| / c+t_{p}+\left\|\mathbf{r}_{t_{i}}-\mathbf{r}_{B, i}\right\| / c+v_{i, T}
$$

where $v_{i, T}$ represents the measurement noise, $c$ is the speed of light, subscript $t_{i}$ is the time at which the measurement from the TRX $i$ is received, and $t_{p}$ is the signal processing time delay. The DOA measurement between the DBS and the $i^{t h}$ TRX is a function of $\mathbf{r}_{i}, \mathbf{r}_{B, i}$, and corresponds to:

$$
\begin{gathered}
\tilde{y}_{i, \theta}=\tan ^{-1} \frac{r_{B, i, y}-r_{t_{i}, y}}{r_{B, i, x}-r_{t_{i}, x}}+v_{i, \theta} \\
\tilde{y}_{i, \phi}=\tan ^{-1} \frac{r_{B, i, z}-r_{t_{i}, z}}{\sqrt{\left(r_{B, i, x}-r_{t_{i}, x}\right)^{2}+\left(r_{B, i, y}-r_{t_{i}, y}\right)^{2}}}+v_{i, \phi}
\end{gathered}
$$

where $\mathbf{r}_{B, i}=\left[\begin{array}{lll}r_{B, i, x} & r_{B, i, y} & r_{B, i, z}\end{array}\right]^{T}, \mathbf{r}_{t_{i}}=\left[\begin{array}{lll}r_{t_{i}, x} & r_{t_{i}, y} & r_{t_{i}, z}\end{array}\right]^{T}, i=1,2, \ldots, N, v_{i, \theta}$ is the 


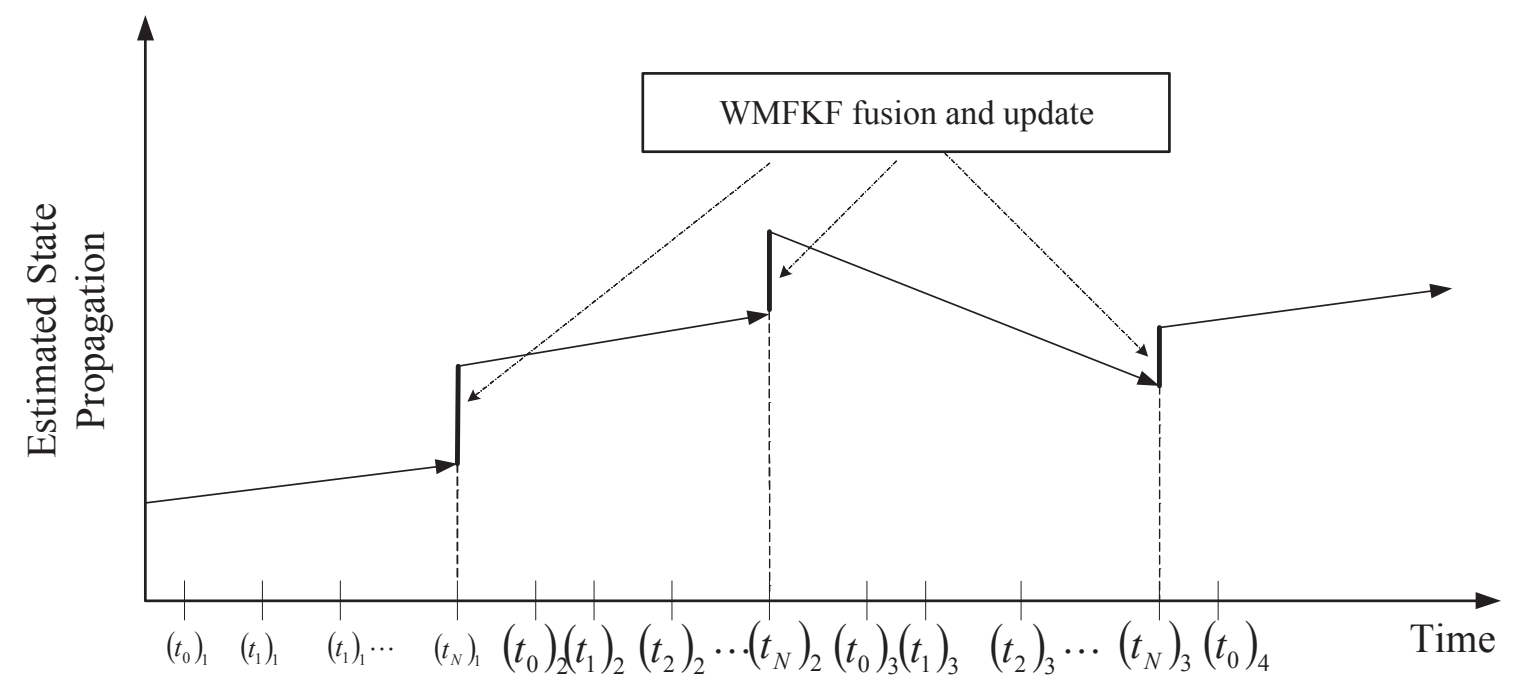

Figure 6.2: WMFKF fuses the measurements and updates the states only at times $\left(t_{N}\right)_{i}$.

relative azimuth angle measurement noise and $v_{i, \phi}$ is the relative elevation angle measurement noise.

The WLPS measurement between the DBS and the $i^{\text {th }}$ TRX in Eqs. (6.1) to (6.3) is represented as $\tilde{\mathbf{y}}_{i}^{p}=\left[\begin{array}{lll}\tilde{y}_{i, T} & \tilde{y}_{i, \theta} & \tilde{y}_{i, \phi}\end{array}\right]^{T}$, with the measurement noise covariance, $R_{i}^{p}=\operatorname{diag}\left\{\left[\begin{array}{lll}v_{i, T}^{2} & v_{i, \theta}^{2} & v_{i, \phi}^{2}\end{array}\right]\right\}$. The term diag represents a diagonal matrix, with its diagonal elements as $v_{i, T}^{2}, v_{i, \theta}^{2}$, and $v_{i, \phi}^{2}$.

For the sake of WMFKF derivation, both the measurement vector, $\tilde{\mathbf{y}}_{i}^{c}$, and the measurement noise covariance, $R_{i}^{c}$, are expressed in Cartesian coordinates: $\tilde{\mathbf{y}}_{i}^{c}=\left[\begin{array}{lll}\tilde{y}_{i, x} & \tilde{y}_{i, y} & \tilde{y}_{i, z}\end{array}\right]^{T}$. It is noted that the measured TOA and DOA in Eqs. (6.1) to (6.3) are functions of the signal transmission delay and the signal processing time delay, as detailed in Ref. [69] and [59]. However, the signal processing time delay is assumed known and the signal transmission time delay is ignored in this WMFKF derivation. Then, the $\tilde{y}_{i, x}, \tilde{y}_{i, y}$ and $\tilde{y}_{i, z}$ are given as:

$$
\begin{gathered}
\tilde{y}_{i, x}=\tilde{y}_{i, D} \cos \left(\tilde{y}_{i, \theta}\right) \cos \left(\tilde{y}_{i, \phi}\right) \\
\tilde{y}_{i, y}=\tilde{y}_{i, D} \sin \left(\tilde{y}_{i, \theta}\right) \cos \left(\tilde{y}_{i, \phi}\right) \\
\tilde{y}_{i, z}=\tilde{y}_{i, D} \sin \left(\tilde{y}_{i, \phi}\right)
\end{gathered}
$$

where $\tilde{y}_{i, D}$ is the distance between $i^{\text {th }}$ TRX and DBS with the assumption of no signal 
transmission time delay, $\tilde{y}_{i, D}=\frac{1}{2}\left(\tilde{y}_{i, T}-t_{p}\right) c$. The transformation from the polar to Cartesian coordinates for the measurement noise covariance is detailed in the Ref. [82], and it is here presented in this functional form:

$$
R_{i}^{c}=g\left(\tilde{y}_{i, D}, \tilde{y}_{i, \theta}, \tilde{y}_{i, \phi}, v_{i, T}, v_{i, \theta}, v_{i, \phi}\right)
$$

\subsection{Weighted Measurement Fusion}

In this section, the WMFKF method is presented. All the WLPS measurements are fused. GPS measurement is not fused. Fig. 6.3 presents a block diagram of the WMFKF algorithm. The WMFKF has a similar propagation and update model as the standard KF. However, all the measurements from all TRXs are fused together into one, before it is applied into the Kalman update process. Considering a linear system, the state models for the state vector, $\mathbf{x}$, and measurement vector, $\tilde{\mathbf{y}}$, are [14]:

$$
\begin{gathered}
\mathbf{x}_{k+1}=\Phi \mathbf{x}_{k}+\mathbf{w} \\
\tilde{\mathbf{y}}=H \mathbf{x}_{k}+v
\end{gathered}
$$

where $\mathbf{w}$ is the process noise vector with a variance $Q=E\left\{\mathbf{w} \mathbf{w}^{T}\right\}$, and $v$ is the measurement noise vector with a variance of $R=E\left\{v v^{T}\right\}$.

For $N$ set of measurements, both $\tilde{\mathbf{y}}$ and $v$ in Eq. (6.9) can be written as $\tilde{\mathbf{y}}=\left[\begin{array}{llll}\left(\tilde{\mathbf{y}}_{1}^{c}\right)^{T} & \left(\tilde{\mathbf{y}}_{2}^{c}\right)^{T} & \ldots & \left(\tilde{\mathbf{y}}_{N}^{c}\right)^{T}\end{array}\right]^{T}$ and $\boldsymbol{v}=\left[\begin{array}{llll}v_{1}^{T} & v_{2}^{T} & \ldots & v_{N}^{T}\end{array}\right]^{T}$ where

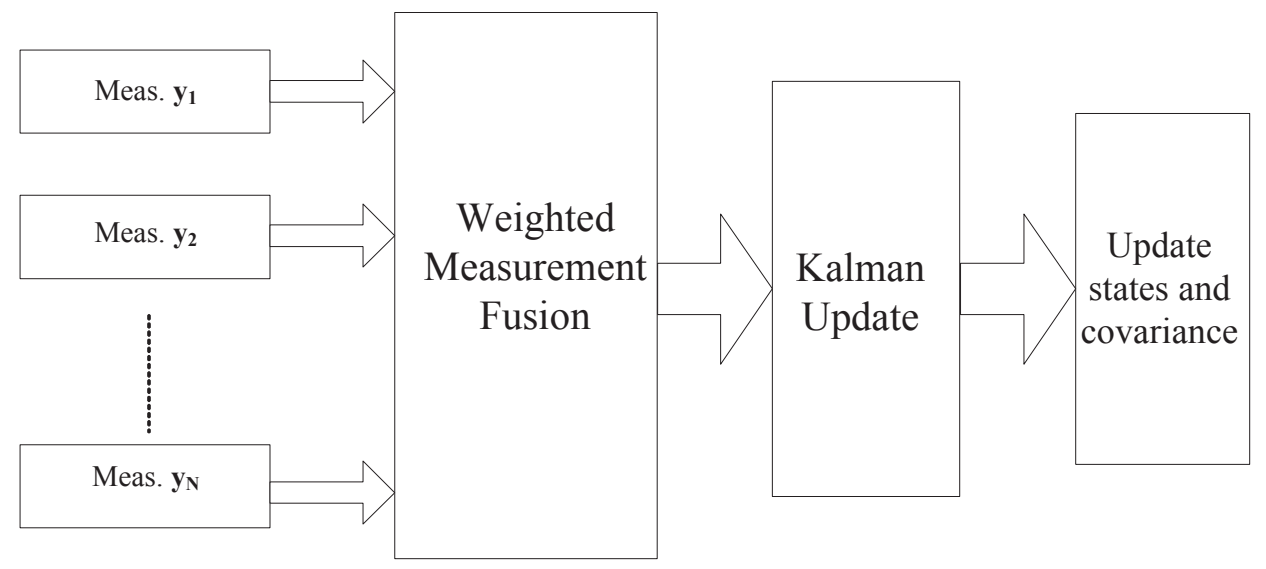

Figure 6.3: Block diagram of the WMFKF algorithm. 
$v_{i}=\left[\begin{array}{lll}v_{i, x} & v_{i, y} & v_{i, z}\end{array}\right]^{T}$, and $v_{i, x}, v_{i, y}$, and $v_{i, z}$ are the measurement noise in the Cartesian coordinates. The fused measurement covariance, $\bar{R}$, and fused measurement vector, $\overline{\mathbf{y}}$ are given as [39]:

$$
\begin{gathered}
\bar{R}=\left(\sum_{i=1}^{N}\left(R_{i}^{c}\right)^{-1}\right)^{-1} \\
\overline{\mathbf{y}}=\bar{R} \sum_{i=1}^{N}\left[\left(R_{i}^{c}\right)^{-1}\left(\mathbf{r}_{B, N / B, i}+\tilde{\mathbf{y}}_{i}^{c}\right)\right]
\end{gathered}
$$

where $\mathbf{r}_{B, N / B, i}=\mathbf{r}_{B, N}-\mathbf{r}_{B, i}, \mathbf{r}_{B, N}$ is the position of the $N^{t h} \mathrm{TRX}$, and $\mathbf{r}_{B, i}$ is the position of $i^{\text {th }}$ TRX. The sensitivity matrix of the fused measurement vector is given as [39]:

$$
\bar{H}=\bar{R} \sum_{i=1}^{N}\left[\left(R_{i}^{c}\right)^{-1} H_{i}\right]
$$

where $H_{i}$ is the corresponding sensitivity matrix of $\tilde{\mathbf{y}}_{i}^{c}$, which is given as:

$$
H_{i}=\left[\begin{array}{ll}
-I_{3 \times 3} & \mathbf{0}_{3 \times 3}
\end{array}\right]
$$

Ref. [39] has analytically shown that the estimation error of the MF method in Eqs. (6.10) and (6.11) is similar to that of the standard KF, in some cases. It has been observed, however, in this study that the estimation error does not always fall within the predicted covariance boundary (or the confidence level of the estimated state error). This situation specifically occurs when any of the TRXs is positioned close to the DBS (e.g. $<1 \mathrm{~km})$. To keep the estimated error within the covariance boundaries, a weighting factor is added during the fusion process.

A weighting factor, $c_{i, y}$, is implemented into the MF method to reduce the convergence rate of the predicted covariance. The $c_{i, y}$ is applied for each measurement vector, $\tilde{\mathbf{y}}_{i}^{c}$, sensitivity matrix, $H_{i}$, and the inverse measurement noise variance, $\left(R_{i}^{c}\right)^{-1}$, in Eqs. (6.10) to (6.12), before they are fused. Therefore, the new fused measurement noise covariance in Eq. (6.10) becomes:

$$
\bar{R}=N\left(\sum_{i=1}^{N} c_{i, y}\left(R_{i}^{c}\right)^{-1}\right)^{-1}
$$

And the new fused measurement vector and sensitivity matrix in Eqs. (6.11) and (6.12) 
become:

$$
\begin{gathered}
\overline{\mathbf{y}}=\left(\sum_{i=1}^{N} c_{i, y}\left(R_{i}^{c}\right)^{-1}\right)^{-1} \sum_{i=1}^{N}\left[c_{i, y}\left(R_{i}^{c}\right)^{-1}\left(\mathbf{r}_{B, N / B, i}+\tilde{\mathbf{y}}_{i}^{c}\right)\right] \\
\bar{H}=\left(\sum_{i=1}^{N} c_{i, y}\left(R_{i}^{c}\right)^{-1}\right)^{-1} \sum_{i=1}^{N}\left[c_{i, y}\left(R_{i}^{c}\right)^{-1} H_{i}\right]
\end{gathered}
$$

The weighting factor, $c_{i, y}$, is a function of the TOAs. The TOA vector, $\mathbf{T}$ is defined as $\mathbf{T}=\left[\begin{array}{llll}\mathbf{y}_{1, T} & \mathbf{y}_{2, T} & \ldots & \mathbf{y}_{N, T}\end{array}\right]^{T}$. The estimated error of standard MF method goes outside the covariance boundaries when a TRX is close to the DBS. In the proposed WMFKF method, the weighting factor for each TRX measurement is assigned based on the distance (or TOA) between TRX and DBS. A higher weight will be assigned to the TRX that is closer the DBS. In other words, the weighting factor, for the $i^{t h} \mathrm{TRX}$, is selected to be inversely proportional to the time difference between the TOA of the $i^{\text {th }}$ TRX. The last measurement, $\mathbf{y}_{N, T}$, which is equivalent to $\max (\mathbf{T})$, has a higher weighting factor than the other measurements. Define the $\Delta T$ and $\tau$ vectors as:

$$
\begin{aligned}
\Delta T_{i} & =\mathbf{y}_{N, T}-\mathbf{y}_{i, T} \\
\tau_{i} & =1-\frac{\Delta \mathbf{T}}{\|\Delta \mathbf{T}\|}
\end{aligned}
$$

where $\Delta \mathbf{T}=\left[\begin{array}{llll}\Delta T_{1} & \Delta T_{2} & \ldots & \Delta T_{N}\end{array}\right]^{T}$, and $\tau=\left[\begin{array}{llll}\tau_{1} & \tau_{2} & \ldots & \tau_{N}\end{array}\right]^{T}$.

Finally, $c_{i, y}$ is defined as:

$$
c_{i, y}=\frac{\tau_{i}^{2}}{\tau^{T} \tau}
$$

Note that $\sum_{i=1}^{N} c_{i, y}=1$. The WMFKF algorithm is similar to that of the standard KF. First, the measurement vectors and measurement noise covariances are fused using the Eqs. (6.14) to (6.16). Then, the estimated state vector and the predicted covariance are updated using the standard KF updates equations developed in Ref. [14]. Then, both the estimated state vector and predicted covariance are propagated to the next time step. 


\subsection{Ellipsoid Error Probable}

The Circular Error Probable (CEP) and the Spherical Error Probable (SEP) [123] have been used extensively in the literature to describe measurement accuracy. Ref. [124] and [125] have presented a two dimensional and three dimensional EEP (E2EP, E3EP) for radionavigation and surveillance systems. The E3EP is used to evaluate the accuracy of the fused measurements, for both the MFKF and the WMFKF.

Given a random vector, $\chi$, where $\chi=(\Delta \mathrm{x}, \Delta \mathrm{y}, \Delta \mathrm{z}), N(0, \sigma)$, and $\Delta \mathrm{x}, \Delta \mathrm{y}$, and $\Delta \mathrm{z}$ are the $\mathrm{x}$, $\mathrm{y}, \mathrm{z}$ components of the position measurement error between DBS and TRX; each $\Delta \mathrm{x}, \Delta \mathrm{y}$, and $\Delta \mathrm{z}$ is assumed to have zero mean errors. The E3EP or the probability that the magnitude of $\chi$ to fall within a magnitude, $\rho$, corresponds to [126]:

$$
P_{\rho}=P\left(\chi^{T} \chi<\rho^{2}\right)=\iiint f(\chi) d \Delta \mathrm{x} d \Delta \mathrm{y} d \Delta \mathrm{z}
$$

where $f(\chi)$ represents the probability density function of $\chi$.

In this chapter, $f(\chi)$ is a function of $\Delta r, \Delta \theta$, and $\Delta \phi$ which are the relative distance error, relative azimuth, and elevation angles error between DBS and TRX respectively. Therefore, Eq. (6.20) can be expressed in polar coordinates as:

$$
P_{\rho}=a_{x} a_{y} a_{z} \int_{0}^{2} \pi \int_{0}^{\phi} \int_{0}^{1} f(\chi) \Delta r^{2} \sin \Delta \theta d \Delta r d \Delta \theta d \Delta \phi
$$

where the $a_{x}, a_{y}$ and $a_{z}$ are the axes of the error ellipsoid.

Eq. (6.21) is computed numerically to determine the EEP. Let $\beta$ be defined as the size increment of the EEP. Then, the $a_{x}, a_{y}$, and $a_{z}$ in Eq. (6.21) can be written as $a_{x}=\beta \sigma_{x}$, $a_{y}=\beta \sigma_{y}$, and $a_{z}=\beta \sigma_{z}$, where $\sigma_{x}, \sigma_{y}$, and $\sigma_{z}$ are the measurement noise standard deviation in Cartesian coordinates presented in Ref. [82]. The EEP is studied with respect to an arbitrary multiplier, $\beta$.

\subsection{Simulation}

Simulations are conducted to compare the performance of the proposed WMFKF and the standard KF for two scenarios: (1) only WLPS measurements are available; and (2) both 
Table 6.1

Position of TRXs.

\begin{tabular}{|c|c|c|c|c|c|}
\hline TRX & Pos. $(\mathrm{X}, \mathrm{Y}, \mathrm{Z}), \mathrm{km}$ & TRX & Pos. $(\mathrm{X}, \mathrm{Y}, \mathrm{Z}), \mathrm{km}$ & TRX & Pos. $(\mathrm{X}, \mathrm{Y}, \mathrm{Z}), \mathrm{km}$ \\
\hline 1 & $1,-1,0.01$ & 9 & $33,30,-0.001$ & 17 & $77,70,-0.001$ \\
2 & $4,5,0.007$ & 10 & $38,31,0.001$ & 18 & $81,73,0.003$ \\
3 & $8,11,0.006$ & 11 & $45,40,0.005$ & 19 & $85,78,0.005$ \\
4 & $13,13,-0.001$ & 12 & $55,50,0.01$ & 20 & $88,77,-0.001$ \\
5 & $17,15,-0.001$ & 13 & $60,55,0.004$ & 21 & $91,80,0.003$ \\
6 & $21,19,0.003$ & 14 & $63,57,0.002$ & 22 & $96,85,0.008$ \\
7 & $24,20,0.01$ & 15 & $68,61,0.001$ & 23 & $101,92,-0.001$ \\
8 & $29,30,0.009$ & 16 & $73,68,0.01$ & 24 & $106,94,0.01$ \\
& & & & 25 & $109,98,0.009$ \\
\hline
\end{tabular}

GPS and WLPS measurements are available. In this simulation, the KF update process only occurs at the time $\left(t_{N}\right)_{i}$ (see Figure 6.2). Also, the KF assumes all signal arrives at time $\left(t_{N}\right)_{i}$. The implementation of KF algorithm can be found in Ref. [22]. The proposed WMFKF algorithm has been presented in Section 6.3.

In the first scenario, the sensitivity matrix, $H$, for the standard KF is $H=\left[\begin{array}{llll}H_{1}^{T} & H_{2}^{T} & \ldots & H_{N}^{T}\end{array}\right]^{T}$, where $H_{i}$ is given in (6.13).

Two detection range limits of WLPS transmission are considered. In the first case, under the Federal Communications Commission (FCC) regulation [127], the detection range of a WLPS is assumed to be $10 \mathrm{~km}$. In the second case, the detection range limit of the WLPS is increased to $30 \mathrm{~km}$. In this simulation, the position of the UAV and all TRXs are expressed with respect to a bearing point, which is located at latitude 45 degrees and longitude 60 degrees, at sea level. The transformation of geodetic coordinate to the Earth Centered Earth Fixed (ECEF) position is presented in Ref. [22]. There are 25 TRXs available along the flight path of the UAV. The position of each TRX is shown in Table 6.1. In addition, we assume that TRX no. 1 is the command center with well-known position, and the other TRXs have a random position error with standard deviation of 1 meter.

In the second scenario, it is assumed that the UAV can detect a maximum 4 GPS signals at the same time. Here, only the $30 \mathrm{~km}$ detection range (second case) is considered. In addition, the WMFKF only fuses the WLPS measurements. The GPS measurement is then processed together with the fused WLPS measurement using a standard KF procedure. Then the KF measurements vector is given as $\tilde{\mathbf{y}}_{W M F K F}=\left[\begin{array}{ll}\tilde{\mathbf{y}}_{G P S}^{T} & \mathbf{y}^{T}\end{array}\right]^{T}$ and 
$\tilde{\mathbf{y}}_{K F}=\left[\begin{array}{ll}\tilde{\mathbf{y}}_{G P S}^{T} & \tilde{\mathbf{y}}^{T}\end{array}\right]^{T}$ respectively. Thus, the sensitivity matrix for the WMFKF and the KF are given as:

$$
\begin{gathered}
H_{W M F K F}=\left[\begin{array}{ll}
H_{G P S}^{T} & \bar{H}^{T}
\end{array}\right]^{T} \\
H_{K F}=\left[\begin{array}{ll}
H_{G P S}^{T} & H^{T}
\end{array}\right]^{T}
\end{gathered}
$$

where the derivation of $\tilde{\mathbf{y}}_{G P S}$ and $H_{G P S}$ can be found in Ref. [22], and the measurement noise covariances are given as:

$$
\begin{gathered}
R_{W M F K F}=\left[\begin{array}{cc}
R_{G P S} & \mathbf{0}_{4 \times 3} \\
\mathbf{0}_{3 \times 4} & \bar{R}
\end{array}\right] \\
R_{K F}=\left[\begin{array}{cc}
R_{G P S} & \mathbf{0}_{4 \times 3 N} \\
\mathbf{0}_{3 N \times 4} & R_{W L P S}
\end{array}\right]
\end{gathered}
$$

where $N$ denotes the number of WLPS within the detection limit range.

The initial position and velocity of the UAV are given as $\mathbf{r}=\left[\begin{array}{lll}0.1 & -0.1 & 9.2\end{array}\right]^{T} \mathrm{~km}$ and $\mathbf{v}=\left[\begin{array}{lll}0.06 & 0.05 & -0.0048\end{array}\right]^{T} k m s^{-1}$, respectively. The estimated state vector is $\hat{\mathbf{x}}=\left[\begin{array}{ll}\mathbf{r}^{T} & \mathbf{v}^{T}\end{array}\right]^{T}$ and the UAV's dynamics is described by the matrix:

$$
\Phi=\left[\begin{array}{cc}
I & \Delta t \times I_{3 \times 3} \\
\mathbf{0}_{3 \times 3} & I_{3 \times 3}
\end{array}\right]
$$

The simulation is run for 30 minutes of flight time. The simulation assumptions for both WMFKF and KF are the same as follow: (1) No signal processing time delay; (2) The standard deviation of measurement noise for TOA is $1 / 3 \times 10^{-6} s$ and for DOA is 0.1 degree; (3) The initial estimated error is a random error of $0.1 \mathrm{~km}$ in position and $1 \mathrm{~ms}^{-1}$ in velocity; (4) The initial state covariance is $1 \mathrm{~km}^{2}$ in position and $0.01 \mathrm{~km}^{2} \mathrm{~s}^{-2}$ in velocity; (5) The WLPS operates at $10 \mathrm{~Hz}$ frequency; (6) There are no multipath effects nor atmospheric delay; (7) The GPS measurement noise standard deviation is $10 \mathrm{~m}$; and (8) There are no clock error and atmosphere delay for the GPS signal. The process noise 

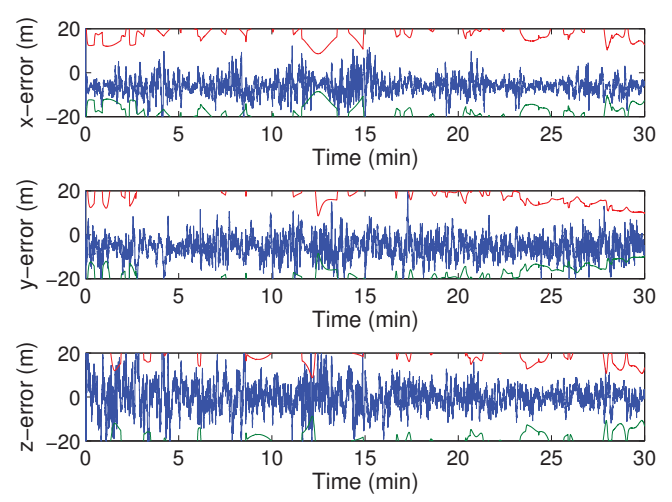

(a) Fusion Kalman Filter.
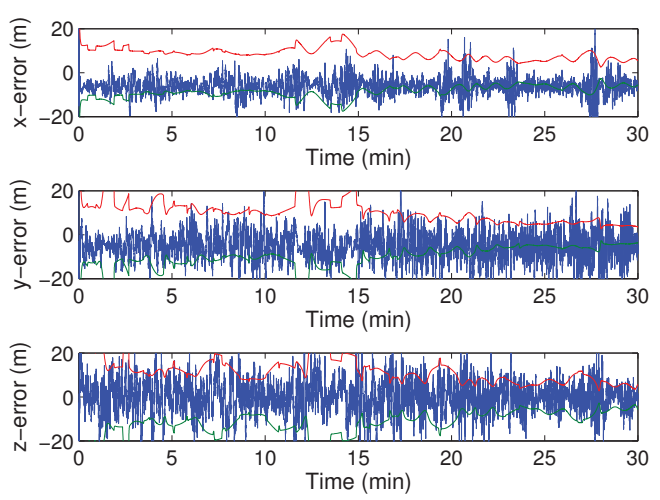

(b) Linear Kalman Filter.

Figure 6.4: Estimated position error (bold solid line) with three sigma boundary (dash-dotted line) plot for $10 \mathrm{~km}$ range limits.

covariance, $Q$, in Eq. (6.8), is given as follow:

$$
Q=\sigma_{v}^{2}\left[\begin{array}{cc}
\frac{1}{3} \Delta t^{3} I_{3 \times 3} & 0.5 \Delta t^{2} I_{3 \times 3} \\
0.5 \Delta t^{2} I_{3 \times 3} & \Delta t I_{3 \times 3}
\end{array}\right]
$$

where $\sigma_{v}=10^{-3} \mathrm{~km} / \mathrm{s}^{3 / 2}$ [128] and $\Delta t$ is the sampling time, which is $0.1 \mathrm{sec}$;

\subsubsection{Scenario One: Accuracy Performance without GPS}

Fig. 6.4(a) and 6.4(b) compare the estimated position error with three sigma boundary for both WMFKF and standard KF. For 10km detection range limit, one to five TRXs always fall within the DBS's coverage area. Fig. 6.4(a) shows that the WMFKF's estimated position error falls within the three sigma boundaries for most of the time; Fig. 6.4(b), however, shows that the KF's estimated position error does not fall within the three sigma boundaries. Similar to the MF method, Fig. 6.4(a) and 6.4(b) show that the WMFKF and the KF have a similar estimation accuracy.

Fig. 6.5(a) and 6.5(b) compare the estimated position error with three sigma boundary for both WMFKF and standard KF for the 30km detection range limit. Fig. 6.5(a) shows that the WMFKF's estimation error always falls within the three sigma boundary. However, Fig. 6.5(b) shows that the KF's estimation error does not always fall within the three 

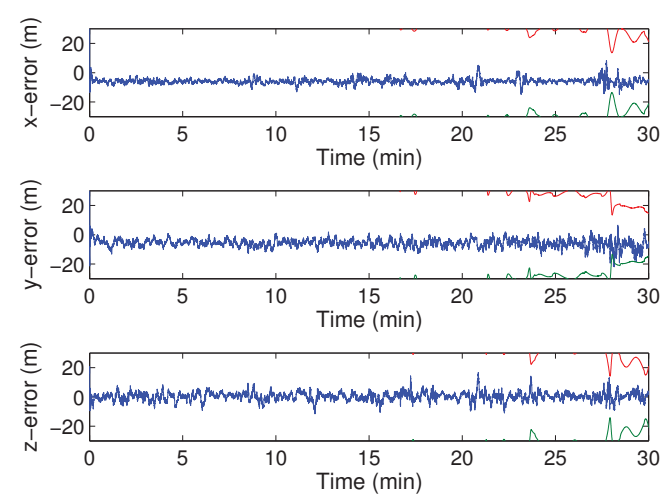

(a) Fusion Kalman Filter.
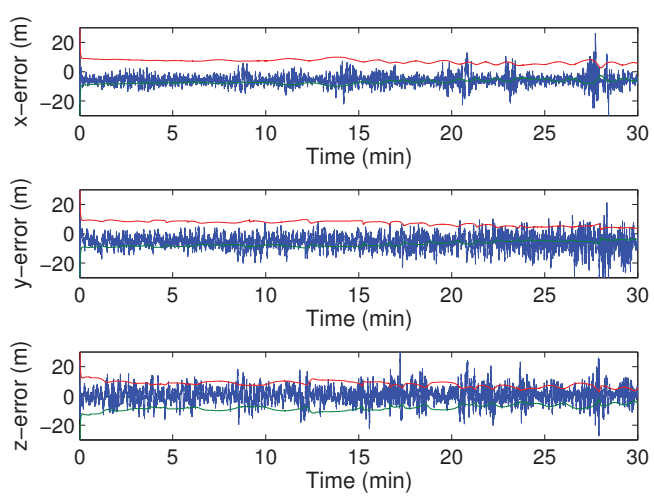

(b) Linear Kalman Filter.

Figure 6.5: Estimated position error (bold solid line) with three sigma boundary (dash-dotted line) plot for $30 \mathrm{~km}$ range limits.

sigma boundary. The results in Fig. 6.4 and 6.5 show that for the linear measurements case, the reduction rate of the fused noise covariance in Eq. (6.10) is proportional to the number of available measurements. The reduction rate of the fused noise covariance has a direct impact on the reduction rate of the predicted covariance. The proposed WMFKF algorithm assigns a higher weight to the measurement (and its covariance) that has lower signal traveling time delay error. Hence, the weight factor in WMFKF can reduce the convergence rate on the predicted covariance. Ref. [40] shows that the fusion algorithm in Eqs. (6.10) to (6.12) is same as the KF in the linear estimation case. This also shows that the KF has a faster convergence rate than the WMFKF in predicted covariance. Also, it should be noted that both WMFKF and KF do not consider the error due to the signal traveling time delay in their estimated measurement and observation matrix models, which results the predicted measurement variance of the KF becomes lower than the actual measurement error variance. Besides that, the results in both Fig. 6.4(a) and 6.5(a) also show that maximum detection range limit affects the effectiveness of the WMFKF method in reducing the convergence rate of the covariance.

\subsubsection{Scenario Two: Accuracy Performance with GPS available}

Fig. 6.6(a) and 6.6(b) compare the estimated position error for both WMFKF and standard $\mathrm{KF}$ for the $30 \mathrm{~km}$ detection range limit with GPS available. Fig. 6.6(a) shows that the 

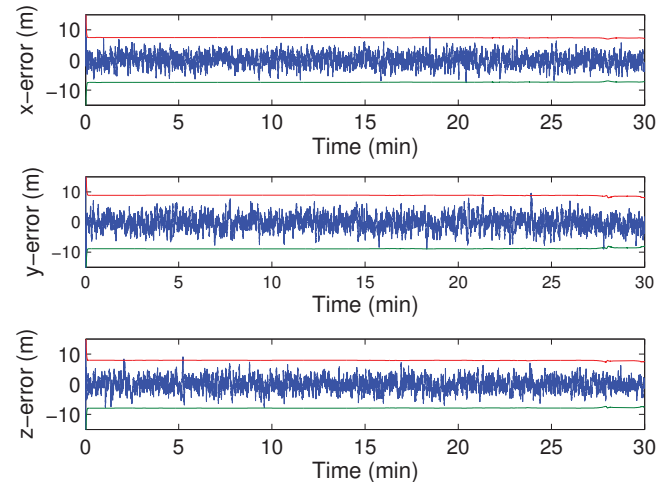

(a) Fusion Kalman Filter.
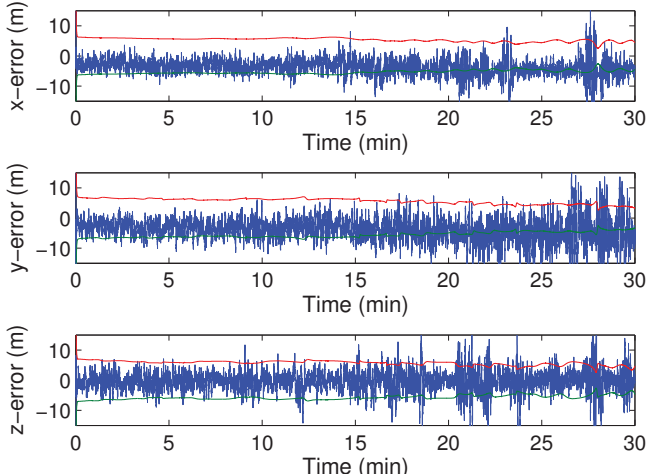

(b) Linear Kalman Filter.

Figure 6.6: Estimated position error (bold solid line) and three sigma boundary (dash-dotted line) plot for $30 \mathrm{~km}$ range limits with GPS available.

WMFKF's estimation error always falls within the three sigma boundary. Although additional GPS measurements are included into the estimation algorithm, Fig. 6.6(b) shows that the KF's estimation error does not fall within the three sigma boundary. Therefore, it can be concluded that additional GPS measurements do not improve the KF's estimation stability. On the other hand, both Fig. 6.6(a) and 6.6(b) show that the WMFKF has a better estimation accuracy than the KF. The WMFKF maintains its estimation error well within the three sigma boundary.

\subsubsection{Ellipsoid Error Probable}

We consider a specific position of the UAV, at $\mathbf{r}=\left[\begin{array}{lll}22 & 19.975 & 7.448\end{array}\right]^{T}$ in scenario one. In addition, we consider that only TRXs no. 2, 3, 5, 6, 7 and 10 in Table 6.1 are available. Three different WLPS detection range limits are considered; these are $10 \mathrm{~km}, 20 \mathrm{~km}$ and $30 \mathrm{~km}$. The given detection range limits also correspond to two, four, and six measurement vectors fusion respectively.

Fig. 6.7 compares the error probability percentage for both the WMFKF method and the MFKF for different numbers of available TRXs. Here, the $\mathrm{x}$-axis represents the size of the error ellipsoid with respect to the fused measurement noise covariance. Fig. 6.7 shows that the WMFKF method has higher EEP compared to the MFKF method, for all cases. In addition, the EEP significantly improves when more TRXs are available for the WMFKF 


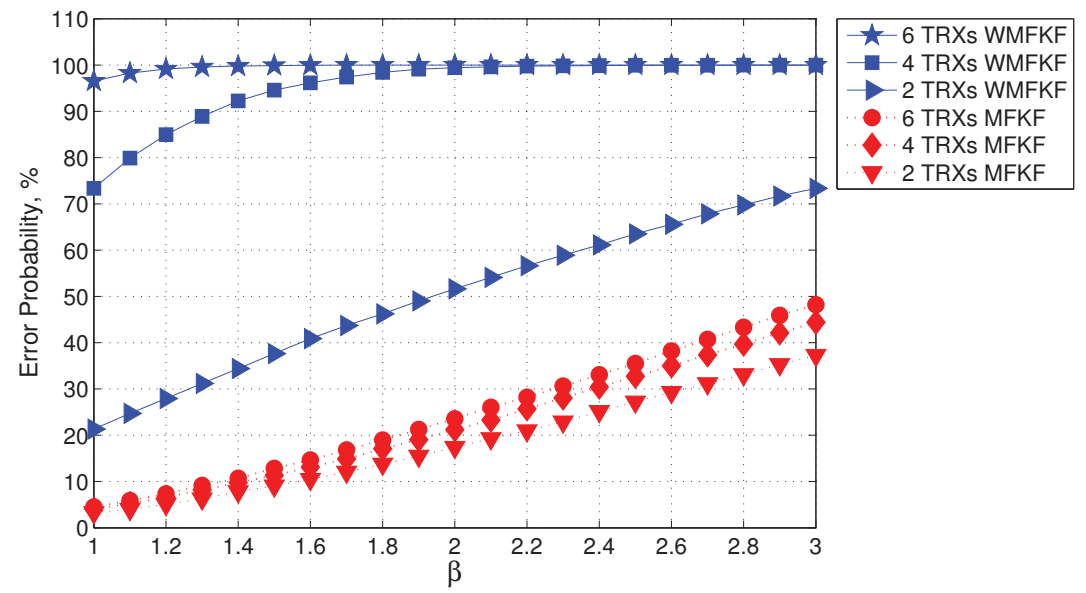

Figure 6.7: Error Probability Percentage for bot the MFKF and the WMFKF.

method. Thus, the WMFKF adjusts the size of the error ellipsoid and increases the confidence level in the measurements error boundaries.

\subsubsection{Comparisons}

There are several modified KF methods for fusing measurements. The following modified KF methods are tested on the above case studies: the Unscented KF (UKF), the state fusion KF method, and algorithm B in reference [120]. In the test cases in this chapter, the system dynamics are linear and the results obtained by the UKF are similar to those of the standard KF. The state fusion KF method and the algorithm B of reference [120] suffer instability due to the high convergence rate of the error covariance.

\subsubsection{Computational Loads}

Table 6.2 compares the number of multiplications required by the WMFKF and the standard KF. Only the GPS denied case study is considered in this computational cost analysis. The $m$ denotes the number of measurements, $N$ denotes the number of TRXs available, where the total number of coordinates in a single measurement is equal to $\mathrm{Nm}$, and $n$ is the total number of estimated states.

For the simulation case presented in this chapter, we have $m=3$, and $n=6$. In the first 
Table 6.2

Comparison of number of multiplication required.

\begin{tabular}{|c|c|c|}
\hline \multirow{2}{*}{ Algorithm } & Number of Multiplication Required \\
\cline { 2 - 3 } & WMFKF & Standard KF \\
\hline$\hat{\mathbf{x}}^{+}=\hat{\mathbf{x}}^{-}+K\left(\tilde{\mathbf{y}}-H \hat{\mathbf{x}}^{-}\right)$ & $n m+m n^{2}$ & $N\left(n m+m n^{2}\right)$ \\
\hline$P^{+}=(I+K H) P$ & $n^{2} m+n^{3}$ & $n^{2} N m+n^{3}$ \\
\hline$K=P^{-} H^{T}\left(H P^{-} H^{T}+R\right)^{-1}$ & $2\left(n^{2} m+n m^{2}\right)+m^{3}+m^{2}$ & $\begin{array}{c}2\left(n^{2} N m+n N m^{2}\right) \\
+N^{3} m^{3}+N^{2} m^{2}\end{array}$ \\
\hline$c_{y}=\tau^{2} /\left(\tau^{T} \tau\right)$ & $5 \mathrm{~N}$ & 0 \\
\hline $\bar{R}=\left(\sum_{i=1}^{N} c_{R} R_{i}^{-1}\right)^{-1}$ & $(N+2) m^{3}+m^{3}$ & 0 \\
\hline$\overline{\mathbf{y}}=\left(\sum_{i=1}^{N} c_{y} R_{i}^{-1}\right)^{-1} \bar{R} \sum_{i=1}^{N} c_{y} R_{i}^{-1} \tilde{\mathbf{y}}_{i}$ & $(N+2) m^{2}+3 * m^{3}$ & 0 \\
\hline
\end{tabular}

case, we assume an average of three TRXs $(N=3)$ are available during the entire simulation. The results show that the standard KF requires 2,700 multiplications for the Kalman update process. However, the WMFKF only requires 1,050 multiplications. For the second case, we assume an average of eight TRXs $(N=8)$ are available during the entire simulation. The results show that the standard KF requires 19,080 multiplications for the Kalman update process, and the WMFKF only requires 1,165 multiplications. The results show that the multiplication required by the standard KF significantly increases as the number of TRXs available increases. However, the WMFKF method avoids this issue. 


\section{Chapter 7}

\section{Conclusion}

The WLPS implementation as a relative position sensor for absolute position estimation in SFF is introduced. It is depicted that 2D WLPS improves positioning accuracy when both WLPS and GPS measurements are used. The results are compared to a GPS standalone system. Moreover, the implementation of 1D WLPS is investigated and simulations are conducted to show the feasibility of obtaining a converging estimation for the absolute positions. The results confirm that the accuracy of 2D WLPS is higher than 1D WLPS. In addition, increasing the number of spacecraft in the formation improves the estimation's convergence time when only relative position measurements are available. Simulation results show, in general, that as the formation size decreases, the WLPS estimation accuracy improves. The impact of formation size becomes significant when either GPS noise is high or GPS measurements are not available. Examples of these situations are deep space missions such as Mars exploration and the Lunar GRAIL mission, and in the low altitude aircraft applications when ground reflection effects are significant. The results also confirm that as the number of spacecraft in the formation increases, a better absolute position estimation performance is attainable.

Chapter three presented an implementation of the Differential Geometric Estimation in relative and absolute positions estimation for spacecraft formations. In the transformed linear domain, two approaches have been implemented in this chapter: the pole placement and the linear filtering approaches. Simulations are conducted to compare the estimation performance of the differential geometric estimation and the extended Kalman filter. The results show that the differential geometric estimation has a faster convergence rate, and 
has better stability, compared to the extended Kalman filter. The complexity analysis shows that the differential geometric estimation has lower complexity when the system is fully observable.

Chapter four presented an implementation of the Constraint Kalman Filter in orbits estimation. Two parameters, $\alpha$ and $\beta$, were introduced in the Constraint Kalman Filter equations to mitigate the impact of the single-point update on the Constrained Kalman Filter process. Two problems were simulated in this chapter. The first problem is the estimation of the absolute spacecraft position from radar measurements. The results show that an improvement of about $25 \%$ (or 5 meters) in the steady state root mean square error can be obtained when implementing the constrained estimation concept presented in this chapter. The second problem is the estimation of formation orbits from measurements of relative positions only. In this problem, the Constraint Kalman Filter performed better than the extended Kalman filter for most of the ranges of $\alpha$ and $\beta$. In the circular orbit case, the Constrained Kalman Filter improves the estimation accuracy up to one fifth of the average root mean square error, as compared to the Extended Kalman Filter. For an elliptic orbit, the Constraint Kalman Filter improves the estimation accuracy by reducing the average root mean square error to one tenth of the Extended Kalman Filter's average root mean square error.

Several parametric studies were conducted to determine best values for the parameters $\alpha$ and $\beta$. The $\beta$ parameter controls how fast the covariance converges due to the application of the constraint while the parameter $\alpha$ controls the amplitude of state update due to the constraint application. From the simulations conducted, it can be concluded that, in general, the $\beta$ value is best if below 0.8 and above the $\alpha$ value. For the best values of $\alpha$ and $\beta$ for a specific problem, a simulation needs to be carried out.

A two-body dynamic model was assumed in all of the cases studied in chapter four. Adding perturbations to the dynamic model is not expected to change the superiority of the constraint estimation to the Extended Kalman Filter. This is due to the fact that, unlike the Extended Kalman Filter, additional information are incorporated in the estimation process in the constraint estimation. The tuning of the $\alpha$ and $\beta$ parameters may become different, though. Additional investigation is needed to study the impact of using perturbed dynamic models on the selection of the parameters $\alpha$ and $\beta$.

The methods to determine the relative attitude between spacecraft in a three spacecraft formation for both parallel and non-parallel cases are presented in chapter five. The 
covariance analysis is carried out to evaluate the level of confidence in the proposed method. The covariance analysis shows that the relative attitude determination errors fall within a three sigma error boundary. The proposed method is capable of providing an accurate and consistent result if the three spacecraft are not close to being colinear.

A Weighted Measurement Fusion Kalman Filter approach is proposed for UAV navigation in chapter six. We assume a UAV is equipped with a remote positioning system called WLPS. The WLPS enables relative localization of the UAV with respect to some bearing points with known positions. The WMFKF and the KF are compared in two cases; the first assumes available GPS measurement and the second assumes a GPS-denied environment. A weighting factor is introduced to reduce the convergence rate of the predicted covariance. Simulation results confirm that the WMFKF has similar accuracy performance as the standard KF when the GPS is not available. However, the weighting method reduces the convergence rate of predicted covariance and ensures that the estimation errors fall within the three sigma boundary. On the other hand, the WMFKF has a better accuracy and stability performance when the GPS measurement is available. In addition, the computational cost of the WMFKF is less than the standard KF. 


\section{Bibliography}

[1] Bernhardt, P. A., Siefring, C., Huba, J., and Selcher, C., "CITRIS: The COSMIC Companion for LEO Radio Occultation," COSMIC Radio Occultation Workshop, Aug. 21, 2002.

[2] Escoubet, C. P., "Cluster-II: Scientific Objectives and Data Dissemination," European Space Agency Bulletin 102, May 2000, pp. 54-60.

[3] Purcell, G., Kuang, D., Lichten, S., Wu, S. C., , and Young, L., "Autonomous Formation Flyer (AFF) Sensor Technology Development,” Feb. 1998, AAS 98-062.

[4] Corazzini, T., Robertson, A., Adams, J. C., Hassibi, A., and How, J. P., "GPS Sensing for Spacecraft Formation Flying," ION-GPS-97 Conference, Sept. 1997.

[5] Psiaki, M. L., "Autonomous Orbit Determination for Two Spacecraft from Relative position Measurements," Journal of Guidance, Control and Dynamics, Vol. 22, No. 2, Mar-Apr 2003.

[6] Yim, J., Crassidis, J., and Junkins, J., "Autonomous Orbit Navigation of Two Spacecraft System Using Relative Line of Sight Vector Measurements," AAS Space Flight Mechanics Meeting, Maui, HI, Feb. 2004.

[7] Kim, S.-G., Crassidis, J. L., Cheng, Y., Fosbury, A. M., and Junkins, J. L., "Kalman Filtering for Relative Spacecraft Attitude and Position Estimation," Aug. 2005, AIAA Paper \#2005-6087.

[8] Folta, D. and Quinn, D., "Enhanced Formation Flying For the Earth Observing-I (EO-I) New Millennium Mission,” Flight Mechanics Symposium 1997, Goddard Space Flight Center, Greenbelt, Maryland, May 19-21, 1997. 
[9] Thales Alenia Space, PROBA 3 ESA Contract 20015/06/NL/JA Formation Flying Demonstration Mission Phase A. Summary Report, 2007.

[10] Vitale, S., LISA Technology Package Architect: The LISA Technology Package on board SMART-2, Oct 2002, ESTEC Contract 15580/01/NL/HB.

[11] McNamara, P., Overview of LISA Pathfinder., 29 April 2009, LISA-LPF-RP-0001.

[12] Andrle, M., Crassidis, J., Linares, R., Cheng, Y., and Hyun, B., "Deterministic Relative Attitude Determination of Three-Vehicle Formations," Journal of Guidance, Control, and Dynamics, Vol. 32, No. 4, 2009, pp. 1077-1088.

[13] Linares, R., Crassidis, J., and Cheng, Y., "Constrained Relative Attitude Determination for Two Vehicle Formations," AIAA Guidance, Navigation, and Control Conference, Chicago, IL, Aug. 2009.

[14] Kalman, R. E., "A New Approach to Linear Filtering and Prediction Problems," Transactions of the ASME-Journal of Basic Engineering, Vol. 82, No. Series D, 1960, pp. 35-45.

[15] Houtekamer, P. and Mitchell, H. L., "A Sequential Ensemble Kalman Filter for Atmospheric Data Assimilation," Monthly Weather Review, Vol. 129, 2001, pp. 123-137.

[16] Goh, S. T., Abdelkhalik, O., and Zekavat, S. A., "Spacecraft Constellation Orbit Estimation via a Novel Wireless Positioning System," 19th AAS/AIAA Space Flight Mechanics Meeting, Savannah, GA, Feb. 2009.

[17] Lee, D. and Pernicka, H., "Vision-Based Relative State Estimation Using the Unscented Kalman Filter," 19th AAS/AIAA Spaceflight Mechanics Meeting, Savannah, Georgia, Feb 9-12, 2009.

[18] Goh, S. T., Unscented Kalman Filtering for Relative Attitude and Position Estimation, Master's thesis, University at Buffalo, Sept. 2007.

[19] Pardal, P., Kuga, H., and de Moraes, R. V., "Robustness Assessment Between Sigma Point and Extended Kalman Filter for Orbit Determination," International Symposium on Space Flight Dynamics, 2011. 
[20] LaViola, J., "A Comparison of Unscented and Extended Kalman filtering for Estimating Quaternion Motion," American Control Conference 2003, June 2003, pp. 2345-2440.

[21] Crassidis, J. and Markley, F., "Unscented Filtering for Spacecraft Attitude Estimation," AIAA Journal of Guidance, Control, and Dynamics, Vol. 26, No. 4, July-Aug. 2003, pp. 536-542.

[22] Crassidis, J. L. and Junkins, J. L., Optimal Estimation of Dynamic Systems, Chapman \& Hall/CRC, Boca Raton, FL, 2004, pp. 243-329.

[23] Simon, D., Optimal State Estimation, John Wiley \& Sons, New York, 2006, pp. 212-223.

[24] Simon, D. and Simon, D., "Constrained Kalman filtering via density function truncation for turbofan engine health estimation," International Journal of Systems Science, Vol. 41, No. 2, Feb. 2010, pp. 159-171.

[25] Porrill, J., "Optimal combination and constraints for geometrical sensor data," International Journal of Robotics Research, Vol. 7(6), Dec 1988, pp. 6677.

[26] Hayward, S., McWhirter, J., and Proudler, I., "Constrained Kalman filter for least-squares estimation of time-varying beamforming weights," Mathematics in Signal Processing IV, Oxford University Press.

[27] Simon, D. and Chia, T., "Kalman filtering with state equality constraints," IEEE Transactions on Aerospace and Electronic Systems, Vol. 38(1), Jan. 2002, pp. $128-136$.

[28] Wen, W. and Durrant-Whyte, H., "Model-based multi-sensor data fusion," IEEE International Conference on Robotics and Automation, May 1992, pp. 1720-1726.

[29] Zanetti, R., Majji, M., Bishop, R. H., and Mortari, D., "Norm-Constrained Kalman Filter," Journal of Guidance, Control and Dynamics, Vol. 32, No. 5, Sept-Oct 2009.

[30] Maybeck, P., Stochastic Models, Estimation, and Control, Vol. 1.

[31] Stengel, R., Optimal Control and Estimation, Dover, New York, 1994. 
[32] Chiang, Y., Wang, L., Chang, F., and Peng, H., "Constrained filtering method for attitude determination using GPS and gyro," IEE Proceedings in Radar, Sonar and Navigation, Oct 2002, pp. 258-264.

[33] Simon, D. and Simon, D., "Kalman filtering with inequality constraints for turbofan engine health estimation," IEE Proceeding in Control Theory and Applications, Vol. 153, May 2006, pp. $371-378$.

[34] Geeter, J. D., Brussel, H. V., Schutter, J. D., and Decreton, M., "A smoothly constrained Kalman filter," IEEE Transactions on Pattern Analysis and Machine Intelligence, Vol. 19, Oct 1997, pp. 1171.

[35] Ran, C. and Deng, Z., "Two correlated measurement fusion Kalman filtering algorithms based on orthogonal transformation and their functional equivalence," Proceedings of the 48th IEEE Conference on Decision and Control, Shanghai, China, Dec. 2009, pp. 2351-2356.

[36] Bar-Shalom, Y. and Campo, L., "The Effect of the Common Process Noise on the Two-Sensor Fused-Track Covariance," IEEE Transactions on Aerospace and Electronic Systems, Vol. 22, No. 6, Nov. 1986, pp. 803-805.

[37] Mosallaei, M. and Salahshoor, R., "Comparison of Centralized Multi-Sensor Measurement and State Fusion Methods with an Adaptive Unscented Kalman Filter for Process Fault diagnosis," 4th International Conference on Information and Automation for Sustainability, 2008., Colombo, Dec. 2008, pp. 519-524.

[38] Roecker, J. and McGillem, C., "Comparison of two-sensor tracking methods based on state vector fusion and measurement fusion," IEEE Transactions on Aerospace and Electronic Systems, Vol. 24, No. 4, Jul 1988, pp. 447-449.

[39] Gan, Q. and Harris, C., "Comparison of Two Measurement Fusion Methods for Kalman-Filter-Based Multisensor Data Fusion," IEEE Transactions on Aerospace and Electronic Systems, Vol. 37, No. 1, Jan 2001, pp. 273-279.

[40] Gao, Y., Ran, C., and Deng, Z., "Weighted measurement fusion Kalman filter based on linear unbiased minimum variance criterion," Proceedings of the 48th IEEE Conference on Decision and Control, Shanghai, China, Dec. 2009, pp. 7593-7598. 
[41] Gao, Y. and Deng, Z., "Reduced dimension measurement fusion Kalman filtering algorithm," 2010 Chinese Control and Decision Conference (CCDC), Xuzhou, China, May 2010, pp. 2184-2188.

[42] Agrachev, A. A. and Gamkrelidze, R. V., "Feedback-invariant optimal control theory and differential geometry.I. Regular extremals," ASME Journal of Dynamical and Control Systems, Vol. 3, 1997, pp. 349-389.

[43] Krishchenko, A. P., Kushnarev, V. I., Nazarenko, A. N., and Tkachev, S. B., "Numerical methods in a differential geometry approach to problems in nonlinear control theory," Journal of Control Theory and Applications, Vol. 29, 1991, pp. 104-114.

[44] Ariff, O., Zbikowski, R., Tsourdos, A., and White, B., "Differential geometric guidance based on the involute of the target's trajectory: 2-D aspect," American Control Conference, 2004, Vol. 4, Boston, MA, 2004, pp. 3640-3645.

[45] Qi;, C. L. W. J. H. W. Z., “Analytical Solution to 3D Differential Geometric Guidance Problem," 1st International Symposium on Systems and Control in Aerospace and Astronautics, Harbin, pp. 652-658.

[46] Kuo, C.-Y., Soetanto, D., and Chiou, Y.-C., "Geometric analysis of flight control command for tactical missile guidance," IEEE Transactions on ontrol Systems Technology, Vol. 9, 2001, pp. 104-114.

[47] Menon, P. K., "Differential Geometric Estimators for Nonlinear Dynamic Systems," AIAA Guidance, Navigation and Control Conference, Honolulu, Hawaii, Aug. 2008.

[48] Lanyi, G., Bagri, D., and Border, J., “Angular Position Determination of Spacecraft by Radio Interferometry," Proceedings of the IEEE, Vol. 95, No. 11, 2007, pp. 2193-2201.

[49] Gunnam, K., Hughes, D., Junkins, J., and Kehtarnavaz, N., "A Vision-Based DSP Embedded Navigation Sensor," IEEE SENSORS JOURNAL, , No. 5, 2002.

[50] Valasek, J., Gunnam, K., Kimmett, J., Tandale, M., and Junkins, J. L., "Vision-Based Sensor and Navigation System for Autonomous Air Refueling," The Journal of Guidance Control and Dynamics, April 2005, pp. 979-989. 
[51] Junkins, J., Hughes, D., Wazni, K., and Pariyapong, V., "Vision-Based Navigation for Rendezvous, Docking and Proximity Operations," 22nd Annual AAS Guidance and Control Conference, Breckenridge, CO, Feb 1999.

[52] Adams, J., Gregorwich, W., Capots, L., and Liccardo, D., "Ultra-wideband for navigation and communications," IEEE Aerospace Conference 2001, Big Sky, MT, March 2001.

[53] Jekeli, C., Inertial Navigation Systems with Geodetic Applications, Walter de Gruyter, New York, 2001.

[54] Hanson, D., "Fundamentals of two-way time transfers by satellite," Proceedings of the 43rd Annual Symposium on Frequency Control, Denver, CO, 1989, pp. 174 178.

[55] Howe, D., Hanson, D., Jespersen, J., Lombardi, M., Klepczynski, W., Wheeler, P., Miranian, M., Powell, W., J, J., and Myers, A., "NIST-USNO time comparisons using two-way satellite time transfers," Proceedings of the 43rd Annual Symposium on Frequency Control, Denver, CO, 1989, pp. 193 - 198.

[56] Kirchner, D., "Two-way time transfer via communication satellites," Proceedings of the IEEE, Jul 1991, pp. $983-990$.

[57] Sun, H. and Li, Y., "Analysis of two way satellite time and frequency transfer system," 9th International Conference on Electronic Measurement and Instruments, Beijing, Aug. 2009, pp. 991-994.

[58] Tong, H. and Zekavat, S. A., "A Novel Wirelss Local Positioning System via a Merger of DS-CDMA and Beamforming: Probability-of-Detection Performance Analysis Under Array Perturbations," IEEE Transactions on Vehicular Technology, Vol. 56, No. 3, May 2007, pp. 1307-1320.

[59] Goh, S. T., Abdelkhalik, O., and Zekavat, S. A., "Spacecraft Formation Orbit Estimation Using WLPS-Based Localization," International Journal of Navigation and Observation, Vol. 2011, 2011.

[60] Goh, S. T., Abdelkhalik, O., and Zekavat, S. A., "Implementation of a Differential Geometric Filter For Spacecraft Formation Orbit Estimation," International Journal of Aerospace Engineering, Accepted. 
[61] Goh, S. T., Abdelkhalik, O., and Zekavat, S. A., "Constraint Estimation of Spacecraft Positions," Journal of Guidance, Control and Dynamics, Vol. 35, No. 2, 2012.

[62] Goh, S., Passerello, C., and Abdelkhalik, O., "Spacecraft Relative Attitude Determination,” 2010 IEEE Aerospace Conference, Big Sky, MT, Mar. 2010.

[63] Goh, S. T., Abdelkhalik, O., and Zekavat, S. A., "A Weighted Measurement Fusion Kalman Filter Implementation for UAV Navigation," Aerospace Science and Technology, Under Review.

[64] Bar-Shalom, Y., "Update with Out of Sequence Measurements in Tracking Exact Solution," IEEE Transactions on Aerospace and Electronic Systems, Vol. 38, No. 3, 2002, pp. 769-777.

[65] Fosbury, A. M., "Satellite Position and Attitude Estimation Using an Extended Kalman Filter," AIAA Guidance, Navigation and Control Conference, Hilton Head, SC, Aug. 2007.

[66] Mason, J., "Algebraic two-satellite TOA/FOA position solution on an ellipsoidal Earth," IEEE Transactions on Aerospace and Electronic Systems, Vol. 40, No. 3, 2004, pp. 1087.

[67] Bayard, D. S., "Reduced-Order Kalman Filtering with Relative Measurements," Journal of Guidance, Control, and Dynamics, Vol. 32, No. 2, 2009, pp. 679-685.

[68] Bar-Shalom, Y., Chen, H., and Mallick, M., "Multistep Out-of-SequenceMeasurement Problem in Tracking," IEEE Transactions on Aerospace and Electronic Systems, Vol. 40, No. 1, 2004, pp. 27-37.

[69] Fosbury, A. M., "Estimation with Multitemporal Measurements," Journal of Guidance, Control, and Dynamics, Vol. 33, No. 5, 2010, pp. 1518-1526.

[70] Zekavat, S. A., Abdelkhalik, O., Ting, S. G., and Fuhrmann, D., “A Novel Space-Based Solar Power Collection via LEO Satellite Networks: Orbital via a Novel Wireless Local Positioning System,” IEEE Aerospace Conference, Big Sky, MT, March 2010.

[71] Zekavat, S. A. and Abdelkhalik, O., "An Introduction to Space-Based Power Grids: Feasibility Study," IEEE Aerospace Conference, Big Sky, MT, March 2011. 
[72] Godara, L., "Application of antenna arrays to mobile communications, part II. Beam-forming and direction-of arrival considerations," Proceedings of the IEEE, Vol. 85, No. 8, Aug 1997, pp. 1195-1245.

[73] Wang, Z. and Zekavat, S. A., "Comparison of Semidistributed Multinode TOA-DOA Fusion Localization and GPS-Aided TOA (DOA) Fusion Localization for MANETs," EURASIP Journal on Advances in Signal Processing, Oct. 2008, pp. 12.

[74] Schaub, H. and Junkins, J. L., Analytical Mechanics of Aerospace Systems, American Institute of Aeronautics and Astronautics, Inc., New York, NY, 2003, p. 680.

[75] Bate, R. R., Mueller, D. D., and White, J. E., Fundamentals of Astroydnamics, Dover, New York, NY, 1971, pp. 20-24, 212-216.

[76] McClain, W. D. and Vallado, D. A., Fundamentals of Astrodynamics and Applications, Springer, 2nd ed., 2001, pp. 26, 52-58, 90-105, 491-674.

[77] Lange, O. and Yang, B., "Optimization of array geometry for direction-of-arrival estimation using a priori information," Advances in Radio Science, 2010, pp. 87-94.

[78] Philip, N. K. and Ananthasayanam, M. R., "Relative position and attitude estimation and control schemes for the final phase of an autonomous docking mission of spacecraft," Acta Astronautica, Vol. 52, No. 7, 2003, pp. 511 - 522.

[79] McTavish, D., Schumacher, R., and Okouneva, G., "Kalman Filtering for Dynamic Pose and Relative Motion Estimation in Orbit," Canadian Aeronautics and Space Journal, , No. 3/4, 2007, pp. 95-105.

[80] Crassidis, J. and Markley, F., "Unscented Filtering for Spacecraft Attitude Estimation," AIAA Journal of Guidance, Control, and Dynamics, Vol. 26, No. 4, July-Aug. 2003, pp. 536-542.

[81] Tong, H., Pourrostam, J., and Zekavat, S. A., "Optimum Beam-forming for a Noise Wireless Local Positioning System: A Stationary Analysis and Solution," EURASIP Journal on Advances in Signal Processing, Vol. 2007, 2007, pp. 12. 
[82] Park, S.-T. and Lee, J. G., "Improved Kalman Filter Design for Three-Dimensional Radar Tracking," IEEE Transactions on Aerospace and Electronic Systems, Vol. 37, No. 2, Apr 2001.

[83] Loan, C. F. V., “Computing Integrals Involving the Matrix Exponential,” IEEE Transactions on Automatic Control, Vol. 23, June 1978, pp. 395-404.

[84] Chen, C.-T., Linear System Theory and Design, Oxford, New York, 1999, pp. 153-158.

[85] Gasparri, A., "Sensor Networks Localization: A computational complexity analysis of an Extended Kalman Filter vs. an Extended Information Filter," Tech. rep., Dipartimento di Informatica ed Automazione (DIA), Universitá degli Studi "Roma Tre”, Via della Vasca Navale, 79 - 00146 Roma, Italy, September 2007.

[86] Edwan, E., Knedlik, S., and O. Loffeld, O., "Constrained Angular Motion Estimation in a Gyro-Free IMU," IEEE Transactions on Aerospace and Electronic Systems, Vol. 47, 2011, pp. 596-610.

[87] Psiaki, M. L., "Attitude-determination filtering via extended quaternion estimation," Journal of Guidance, Control and Dynamics, Vol. 23, No. 2, 2000, pp. 206-214.

[88] Zanetti, R. and Bishop, R. H., "Quaternion Estimation and Norm Constrained Kalman Filtering," AIAA/AAS Astrodynamics Specialist Conference and Exhibit, Keystone, Colorado, Aug. 21-24, 2006.

[89] Li, W. and Leung, H., "Constrained unscented Kalman filter based fusion of GPS/INS/digital map for vehicle localization," IEE Proceedings in Intelligent Transportation Systems, 2003, pp. 1362-1367.

[90] Wang, J.-H. and Y. Gao, Y., "Land Vehicle Dynamics-Aided Inertial Navigation," IEEE Transactions on Aerospace and Electronic Systems, Vol. 46, No. 4, 2010.

[91] Hill, K., Born, G., and M.W.Lo, "Linked, Autonomous, Interplanetary Satellite Orbit Navigation (LiAISON) in Lunar Halo Orbits," AAS/AIAA Astrodynamics Specialist Conference.

[92] Yim, J., Crassidis, J., and Junkins, J., “Autonomous Orbit Navigation of Two Spacecraft System Using Relative Line of Sight Vector Measurements," AAS Space Flight Mechanics Meeting, Maui, HI, Feb 2004. 
[93] Wang, Z. and Zekavat, S. A., "MANET Localization via Multi-node TOA-DOA Optimal Fusion," proceedings IEEE Milcom 2006, Washington DC, Oct. 2006.

[94] Walker, D. M., "Parameter estimation using Kalman filters with constraints," International Journal of Bifurcation and Chaos, Vol. 16, No. 4, 2006, pp. 1067-1078.

[95] Kriegsman, B. and Tao, Y., "Shuttle Navigation System for Entry and Landing Mission Phases," Journal of Spacecraft and Rockets, Vol. 12, No. 4, 1975, pp. 213-219.

[96] Anderson, E., Bai, Z., Bischof, C., Blackford, S., Demmel, J., Dongarra, J., Croz, J. D., Greenbaum, A., Hammarling, S., McKenney, A., and Sorensen, D., LAPACK Users' Guide, Society for Industrial and Applied Mathematics, Philadelphia, PA, 3rd ed., 1999.

[97] Skolnik, M. I., “Tracking Radar,” Radar Handbook, chap. 9, McGraw-Hill, 2008.

[98] Wertz, J. and Larson, W., Space Mission Analysis and Design, 1999.

[99] Cohen, C., Global Positioning System: Theory and Applications, Vol. II, AIAA, New York, 1996.

[100] Kim, S.-G., "Kalman Filtering for Relative Spacecraft Attitude and Position Estimation," May 1 2005, master thesis.

[101] Geller, D., "Analysis of the Relative Attitude Estimation and Control Problem for Satellite Inspection and Orbital Rendezvous," The Journal of the Astronautical Sciences, Vol. 55, No. 2, Apr-June 2007.

[102] Bortoluzzi, D., Hoyle, C., Hueller, M., Vitale, S., Heinzel, G., Danzmann, K., Barcelona, A. L. I., Anza, S., Navau, C., Chen, D.-X., Sanchez, A., Araujo, H., Wass, P., and Grimani, C., LISA Technology Package Architect: Science Requirements and Top-level Architecture Definition for the Lisa Technology Package (LTP) on Board LISA Pathfinder (SMART-2), June 2005, LTPA-UTN-ScRD-Iss003-Rev1.

[103] Shuster, M. and OH, S., "Three-Axis Attitude Determination from Vector Observations," Journal of Guidance, Control, and Dynamics, Vol. 4, No. 1, 1981, pp. 70-77. 
[104] Shuster, M., "A Broad Look at Deterministic Three-Axis Attitude Determination," AIAA/AAS Astrodynamics Specialists Conference, Denver, CO, Aug. 2000.

[105] Wahba, G., "A Least Squares Estimate of Spacecraft Attitude," SIAM Review, Vol. 7, No. 3, July 1965 , pp. 409.

[106] Farrell, J. and Stuelpnagel, J., "Solution to Problem 65-1," SIAM Review, Vol. 8, July 1966, pp. 384-386.

[107] Pisacane, V., Fundamentals of Space Systems, Oxford University Press, New York, 2nd ed., 2005.

[108] Mortari, D., "Optimal Cones Intersection Technique for Attitude Pointing Error Evaluation," Advances in the Astronautical Sciences, Vol. 97, 1997, pp. 949-962.

[109] Mortari, D., "EULER-2 and EULER-n Algorithm for Attitude Determination from Vector Observations," Space Technology, Vol. 16, No. 5/6, 1996, pp. 317-321.

[110] Shuster, M. and Oh, S., "Attitude Determination from Vector Observations," The Journal of Guidance and Control, Vol. 4, No. 1, Jan - Feb 1981, pp. 70-77.

[111] Markley, F., "Attitude Determination from Vector Observations: A Fast Optimal Matrix Algorithm," The Journal of the Astronautical Sciences, Vol. 41, No. 2, April 1993, pp. 261-280.

[112] Mortari, D., "ESOQ: A Closed-Form Solution to the Wahba Problem," Journal of the Astronautical Sciences, Vol. 45, April-June 1997, pp. 195-204.

[113] Shuster, M., "Maximum Likelihood Estimation of Spacecraft Attitude," The Journal of the Astronautical Sciences, Vol. 37, No. 1, Jan-March 1989, pp. 79-88.

[114] Lefferts, E., Markley, F., and Shuster, M., "Kalman Filtering for Spacecraft Attitude Estimation," Journal of Guidance, Control, and Dynamics, Vol. 5, No. 5, Sept-Oct. 1982, pp. 417-429.

[115] Singla, P., Griffith, T. D., Crassidis, J. L., and Junkins, J. L., “Attitude Determination and Autonomous On-orbit Calibration of Star Tracker for GIFTS Mission," AAS/AIAA Spaceflight Mechanics Meeting, Vol. 112, 27-30 January, 2002. 
[116] Fallon, L., chap. Star Sensors, Kluwer Academic Publishers, The Netherlands, 1978, pp. 184-195.

[117] Kim, J. and Sukkarieh, S., "Autonomous airborne navigation in unknown terrain environments," IEEE Transactions on Aerospace and Electronic Systems, Vol. 40, No. 3, 2004, pp. 1031-1045.

[118] Farrell, J. and Barth, M., The Global Positioning System and Inertial Navigation, chap. 1, McGraw-Hill Professional, 1989, pp. 1-20.

[119] Kim, J. and Sukkarieh, S., "SLAM aided GPS/INS Navigation in GPS Denied and Unknown Environments," The 2004 International Symposium on GNSS/GPS, Sydney, Australia, December 2004.

[120] Hilton, R., Martin, D., and Blair, W., “Tracking with time-delayed data in multisensor systems," Tech. rep., NAVAL SURFACE WARFARE CENTER DAHLGREN DIV VA, Dahlgren, VA, Aug 1993, NSWCDD/TR-93/351.

[121] Mallick, M. and Marrs, A., "Comparison on of the KF and Particle Filter Based Out-of-Sequence Measurement Filtering Algorithms," Proc. Sixth International Conference Information Fusion, Vol. 1, pp. 422-429.

[122] Kwok, C., Fox, D., and Meila, M., "Real-time particle filters," Proceedings of the IEEE, Vol. 92, 2004, pp. 469-484.

[123] Marco, S. D. and Raytheon, T., "A series representation of the spherical error probability integral," IEEE Transactions on Aerospace and Electronic Systems, Vol. 29, 1993, pp. 1349 - 1356.

[124] Luzica, S., P.Svoboda, and Grecman, P., "Mode-S positional data error analysis from the point of view of air traffic control," No. 30, 2006.

[125] Luzica, S. and Grecman, P., "Radionavigation and surveillance systems: Positioning error value evaluation of in Matlab Programming enviroment," Proceedings of the International Conference on Military Technologies 2009, University of Defense, Czech, May 2009, pp. 430-437.

[126] Gillis, J., "Computation of the circular error probability integral," IEEE Transactions on Aerospace and Electronic Systems, Vol. 27, 1991, pp. 906-910. 
[127] Commission, F. C., A Local Government Official's Guide toTransmitting Antenna RF Emission Safety:Rules, Procedures, and Practical Guidance, June 2000.

[128] Jwo, D.-J. and Wang, S.-H., "Adaptive Fuzzy Strong Tracking Extended Kalman Filtering for GPS Navigation," IEEE SENSORS JOURNAL, Vol. 7, No. 5, May 2007, pp. 778-789. 


\section{Chapter 8}

\section{Appendix}

\subsection{Linearization and covariance analysis in polar and cartesian coordinate}

\subsubsection{Time derivative of state vectors in polar and cartersian coordinate}

For simplicity, in this section, we assume $r \equiv r_{i}, \lambda \equiv \lambda_{i}$ and $\xi \equiv \xi_{i}$.

1. First order time derivatives of polar coordinates is:

$$
\begin{gathered}
\dot{r}=\frac{x \dot{x}+y \dot{y}+z \dot{z}}{r} \\
\dot{\lambda}=\frac{x \dot{y}-y \dot{x}}{x^{2}+y^{2}} \\
\dot{\xi}=\frac{\dot{z}\left(x^{2}+y^{2}\right)^{1 / 2}}{r^{2}}-\frac{z(x \dot{x}+y \dot{y})}{r^{2}\left(x^{2}+y^{2}\right)^{1 / 2}}
\end{gathered}
$$

2. The Cartesian representation in polar coordinates is:

$$
\begin{gathered}
x=r \cos \lambda \cos \xi \\
y=r \sin \lambda \cos \xi \\
z=r \sin \xi
\end{gathered}
$$


3. First order time derivatives of cartesian coordinates is:

$$
\begin{gathered}
\dot{x}=\dot{r} \cos \lambda \cos \xi-r \dot{\lambda} \sin \lambda \cos \xi-r \dot{\xi} \cos \lambda \sin \xi \\
\dot{y}=\dot{r} \sin \lambda \cos \xi+r \dot{\lambda} \cos \lambda \sin \xi-r \dot{\xi} \sin \lambda \sin \xi \\
\dot{z}=\dot{r} \sin \xi+r \dot{\xi} \cos \xi
\end{gathered}
$$

\subsubsection{Conversion of measurement noise covariance from polar to cartesian coordinate}

Ref. [82] shows the expression cartesian coordinate measurement noise in terms of polar coordinate vector. Given that $r$ is the relative range, $\psi$ and $\phi$ are the relative elevation and azimuth angles at the current time step respectively. The corresponding measurement noise in standard deviation are $\sigma_{r}, \sigma_{\psi}$ and $\sigma_{\phi}$ respectively. Then, the measurement noise covariance, $\mathfrak{R}_{i j}$, in cartesian coordinate is given as:

$$
\begin{aligned}
\mathfrak{R}_{i j}=T_{k} \operatorname{diag} & \left\{\left(r^{2}+\sigma_{r}^{2}\right)\left(1+e^{-2 \sigma_{\psi}^{2}}\right)\left(1+e^{-2 \sigma_{\phi}^{2}}\right) / 4-r^{2} e^{-} \sigma_{\psi}^{2}+\sigma_{\phi}^{2},\right. \\
& \left(r^{2}+\sigma_{r}^{2}\right)\left(1-e^{-2 \sigma_{\psi}^{2}}\right)\left(1+e^{-2 \sigma_{\phi}^{2}}\right) / 4 \\
& \left(r^{2}+\sigma_{r}^{2}\right)\left(1+e^{-2 \sigma_{\psi}^{2}}\right)\left(1-e^{-2 \sigma_{\phi}^{2}}\right) / 4 \\
& \left.\left(r^{2}+\sigma_{r}^{2}\right)\left(1-e^{-2 \sigma_{\psi}^{2}}\right)\left(1-e^{-2 \sigma_{\phi}^{2}}\right) / 4\right\} T_{k}^{T}
\end{aligned}
$$

where the "diag" represents the diagonal matrix, and $T_{k}$ is given as:

$$
T_{k}=\left[\begin{array}{cccc}
\cos \psi \cos \phi & -\sin \psi \cos \phi & -\cos \psi \sin \phi & \sin \psi \sin \phi \\
\sin \psi \cos \phi & \cos \psi \cos \phi & -\sin \psi \sin \phi & \cos \psi \sin \phi \\
\sin \phi & 0 & -\cos \phi & 0
\end{array}\right]
$$




\section{Chapter 9}

\section{Appendix}

\subsection{Linearization of orbital elements, and $f$ and $g$ function}

\subsubsection{Partial Derivatives for semimajor axis, eccentricity, and eccentric anomaly}

The semimajor axis, eccentricity, and eccentric anomaly are nonlinear functions of the spacecraft's absolute position vector, $\mathbf{r}$, and the velocity vector $\mathbf{v}[75,76]$. For simplicity, we assume $\mathbf{r} \equiv \mathbf{r}_{i, k}$ and $\mathbf{v} \equiv \mathbf{v}_{i, k}$ in Appendix section. According to Eqs. (4.19) and (4.20), the eccentric anomaly and eccentricity are required to determined both $\Delta t_{a}$ and $\Delta t_{p}$, to construct both $f$ and $g$ functions. The partial derivative (or linearization) of eccentric anomaly and eccentricity with respect to spacecraft absolute position and velocity vectors are presented below.

The partial derivatives of eccentricity with respect to position and velocity vectors are:

$$
\begin{aligned}
\frac{\partial e}{\partial \mathbf{r}} & =\frac{\mathbf{e}^{T}}{\sqrt{\mathbf{e}^{T} \mathbf{e}}} \frac{\partial \mathbf{e}}{\partial \mathbf{r}} \\
\frac{\partial e}{\partial \mathbf{v}} & =\frac{\mathbf{e}^{T}}{\sqrt{\mathbf{e}^{T} \mathbf{e}}} \frac{\partial \mathbf{e}}{\partial \mathbf{v}}
\end{aligned}
$$


where,

$$
\begin{gathered}
\frac{\partial \mathbf{e}}{\partial \mathbf{r}}=\frac{\|\mathbf{v}\|^{2} \times I_{3 \times 3}}{\mu}-\frac{\mathbf{v v}^{T}}{\mu}-\frac{I_{3 \times 3}}{\|\mathbf{r}\|}+\frac{\mathbf{r r}^{T}}{\|\mathbf{r}\|^{3}} \\
\frac{\partial \mathbf{e}}{\partial \mathbf{v}}=\frac{2\|\mathbf{v}\| \mathbf{r} \mathbf{v}^{T}}{\mu}-\frac{\mathbf{r}^{T} \mathbf{v} \times I_{3 \times 3}}{\mu}-\frac{\mathbf{v r}^{T}}{\mu}
\end{gathered}
$$

and $I_{3 \times 3}$ is a 3 by 3 identity matrix.

The partial derivative of eccentric anomaly with respect to the position and velocity vectors are:

$$
\begin{aligned}
\frac{\partial E}{\partial \mathbf{r}}= & \frac{1}{1+\left(\frac{\sqrt{1-e^{2}} \sin \theta}{e+\cos \theta}\right)^{2}}\left\{\frac{\sqrt{1-e^{2}} \cos \theta}{e+\cos \theta} \frac{\partial \theta}{\partial \mathbf{r}}-\frac{\sin \theta}{\sqrt{1-e^{2}} \cos \theta} \frac{\partial \mathbf{e}}{\partial \mathbf{r}}\right. \\
& \left.-\frac{\sqrt{1-e^{2}} \sin \theta}{(e+\cos \theta)^{2}}\left(\frac{\partial \mathbf{e}}{\partial \mathbf{r}}-\sin \theta \frac{\partial \theta}{\partial \mathbf{r}}\right)\right\} \\
\frac{\partial E}{\partial \mathbf{v}}= & \frac{1}{1+\left(\frac{\sqrt{1-e^{2}} \sin \theta}{e+\cos \theta}\right)^{2}}\left\{\frac{\sqrt{1-e^{2}} \cos \theta}{e+\cos \theta} \frac{\partial \theta}{\partial \mathbf{v}}\right. \\
& \left.-\frac{\sin \theta}{\sqrt{1-e^{2}} \cos \theta} \frac{\partial \mathbf{e}}{\partial \mathbf{v}}-\frac{\sqrt{1-e^{2}} \sin \theta}{(e+\cos \theta)^{2}}\left(\frac{\partial \mathbf{e}}{\partial \mathbf{v}}-\sin \theta \frac{\partial \theta}{\partial \mathbf{v}}\right)\right\}
\end{aligned}
$$

where the partial derivatives of the true anomaly are:

$$
\begin{gathered}
\frac{\partial \theta}{\partial \mathbf{r}}=\frac{-1}{\sqrt{1-\left(\frac{\mathbf{e}^{T} \mathbf{r}}{\|\mathbf{e}\|\|\mathbf{r}\|}\right)^{2}}}\left\{\frac{\mathbf{e}^{T}}{\|\mathbf{e}\|\|\mathbf{r}\|}-\frac{\mathbf{e}^{T} \mathbf{r r}^{T}}{\|\mathbf{e}\|\|\mathbf{r}\|^{3}}+\left(\frac{\mathbf{r}^{T}}{\|\mathbf{e}\|\|\mathbf{r}\|}-\frac{\mathbf{r}^{T} \mathbf{e e}^{T}}{\|\mathbf{e}\|^{3}\|\mathbf{r}\|}\right) \frac{\partial \mathbf{e}}{\partial \mathbf{r}}\right\} \\
\frac{\partial \theta}{\partial \mathbf{v}}=\frac{-1}{\sqrt{1-\left(\frac{\mathbf{e}^{T} \mathbf{r}}{\|\mathbf{e}\|\|\mathbf{r}\|}\right)^{2}}}\left\{\left(\frac{\mathbf{r}^{T}}{\|\mathbf{e}\|\|\mathbf{r}\|}-\frac{\mathbf{r}^{T} \mathbf{e e}^{T}}{\|\mathbf{e}\|^{3}\|\mathbf{r}\|}\right) \frac{\partial \mathbf{e}}{\partial \mathbf{v}}\right\}
\end{gathered}
$$

The partial derivatives of semimajor axis with respect to position and velocity vectors are:

$$
\begin{gathered}
\frac{\partial a}{\partial \mathbf{r}}=\frac{2 \mu^{2} \mathbf{r}^{T}}{\|\mathbf{r}\|\left(2 \mu-\|\mathbf{r}\|\|\mathbf{v}\|^{2}\right)^{2}} \\
\frac{\partial a}{\partial \mathbf{v}}=\frac{2 \mu\|\mathbf{r}\| \mathbf{v}^{T}}{\left(2 \mu-\|\mathbf{r}\|\|\mathbf{v}\|^{2}\right)^{2}}
\end{gathered}
$$




\subsubsection{Linearization of $\Delta t_{p}$ and $\Delta t_{a}$.}

The $\Delta t_{a}$ and $\Delta t_{p}$ in Eqs. (4.19) and (4.20) are expressed in terms of $a, e$ and $E$. Let $\Delta t$ represent both $\Delta t_{a}$ and $\Delta t_{p}$, the partial derivatives of $\Delta t$ with respect to a spacecraft's absolute position and velocity vectors are:

$$
\begin{aligned}
& \frac{\partial \Delta t}{\partial \mathbf{r}}=\sqrt{\frac{a^{3}}{\mu}}\left(-\frac{\partial E}{\partial \mathbf{r}}+\sin E \frac{\partial e}{\partial \mathbf{r}}+e \cos E \frac{\partial E}{\partial \mathbf{r}}\right)+\frac{3}{2} \sqrt{\frac{a}{\mu}}\left[E_{a p}-(E-e \sin E)\right] \frac{\partial a}{\partial \mathbf{r}} \\
& \frac{\partial \Delta t}{\partial \mathbf{v}}=\sqrt{\frac{a^{3}}{\mu}}\left(-\frac{\partial E}{\partial \mathbf{v}}+\sin E \frac{\partial e}{\partial \mathbf{v}}+e \cos E \frac{\partial E}{\partial \mathbf{v}}\right)+\frac{3}{2} \sqrt{\frac{a}{\mu}}\left[E_{a p}-(E-e \sin E)\right] \frac{\partial a}{\partial \mathbf{v}}
\end{aligned}
$$

where the partial derivatives for $a, e$ and $E$ are shown in Eqs. (9.1) to (9.10), $E_{a p}=0$ for $\Delta t \equiv \Delta t_{p}$, and $E_{a p}=\pi$ for $\Delta t \equiv \Delta t_{a}$.

\subsubsection{Linearization of $f$ and $g$ functions.}

The derivation of $\frac{d g(\Delta t, \mathbf{r}, \mathbf{v})}{d \mathbf{r}}$ and $\frac{d g(\Delta t, \mathbf{r}, \mathbf{v})}{d \mathbf{v}}$ in Eqs. (4.23) and (4.24) are here presented. The $f, g, \dot{f}$ and $\dot{g}$, in Universal Variables [76], are:

$$
\begin{gathered}
f=1-\frac{\chi^{2}}{\|\mathbf{r}\|} c_{2} \\
g=\Delta t-\frac{\chi^{3}}{\sqrt{\mu}} c_{3} \\
\dot{f}=\frac{\sqrt{\mu}}{\|\mathbf{r}\| R} \chi\left(\psi c_{3}-1\right) \\
\dot{g}=1-\frac{\chi^{2}}{R} c_{2}
\end{gathered}
$$

where $R, c_{2}, c_{3}$ and $\psi$ are constant variables (detailed in Ref. [76]), and $\chi$ is:

$$
\chi=\sqrt{\mu} \gamma \Delta t
$$

and,

$$
\gamma=\frac{-\|\mathbf{v}\|^{2}}{\mu}+\frac{2}{\|\mathbf{r}\|}
$$


Both $\frac{d g(\Delta t, \mathbf{r}, \mathbf{v})}{d \mathbf{r}}$ and $\frac{d g(\Delta t, \mathbf{r}, \mathbf{v})}{d \mathbf{v}}$ are given as:

$$
\begin{aligned}
\frac{d g(\Delta t, \mathbf{r}, \mathbf{v})}{d \mathbf{r}} & =\left(\frac{\dot{f}}{D U^{2}} \mathbf{r}^{T} \mathbf{r}+\dot{g} \frac{T U}{D U^{2}} \mathbf{r}^{T} \mathbf{v}\right) \frac{\partial f}{\partial \mathbf{r}}+\left(\frac{f}{D U^{2}} \mathbf{r}^{T} \mathbf{r}+g \frac{T U}{D U^{2}} \mathbf{r}^{T} \mathbf{v}\right) \frac{\partial \dot{f}}{\partial \mathbf{r}} \\
& +\left(\dot{g} \frac{T U^{2}}{D U^{2}} \mathbf{v}^{T} \mathbf{v}+\dot{f} \frac{T U}{D U^{2}} \mathbf{r}^{T} \mathbf{v}\right) \frac{\partial g}{\partial \mathbf{r}}+\left(g \frac{T U^{2}}{D U^{2}} \mathbf{v}^{T} \mathbf{v}+f \frac{T U}{D U^{2}} \mathbf{r}^{T} \mathbf{v}\right) \frac{\partial \dot{g}}{\partial \mathbf{r}} \\
& -\frac{\mathbf{r}_{i, a / p}^{T} \mathbf{v}_{i, a / p}}{\left\|\mathbf{r}_{i, a / p}\right\|^{3}}\left[\left(f \mathbf{r}^{T} \mathbf{r}+g \mathbf{r}^{T} \mathbf{v}\right) \frac{\partial f}{\partial \mathbf{r}}+\left(f \mathbf{r}^{T} \mathbf{v}+g \mathbf{v}^{T} \mathbf{v}\right) \frac{\partial g}{\partial \mathbf{r}}\right] \\
\frac{d g(\Delta t, \mathbf{r}, \mathbf{v})}{d \mathbf{v}}= & \left(\frac{\dot{f}}{D U^{2}} \mathbf{r}+\dot{g} \frac{T U}{D U^{2}} \mathbf{r}^{T} \mathbf{v}\right) \frac{\partial f}{\partial \mathbf{v}}+\left(\frac{f}{D U^{2}} \mathbf{r}^{T} \mathbf{r}+g \frac{T U}{D U^{2}} \mathbf{r}^{T} \mathbf{v}\right) \frac{\partial \dot{f}}{\partial \mathbf{v}} \\
+ & \left(\frac{T U^{2}}{D U^{2}} \mathbf{v}^{T} \mathbf{v}+\dot{f} \frac{T U}{D U^{2}} \mathbf{r}^{T} \mathbf{v}\right) \frac{\partial g}{\partial \mathbf{v}}+\left(g \frac{T U^{2}}{D U^{2}} \mathbf{v}^{T} \mathbf{v}+f \frac{T U}{D U^{2}} \mathbf{r}^{T} \mathbf{v}\right) \frac{\partial \dot{g}}{\partial \mathbf{v}} \\
& -\frac{\mathbf{r}_{i, a / p}^{T} \mathbf{v}_{i, a / p}\left[\left(f \mathbf{r}^{T} \mathbf{r}+g \mathbf{r}^{T} \mathbf{v}\right) \frac{\partial f}{\partial \mathbf{v}}+\left(f \mathbf{r}^{T} \mathbf{v}+g \mathbf{v}^{T} \mathbf{v}\right) \frac{\partial g}{\partial \mathbf{v}}\right]}{\left\|\mathbf{r}_{i, a / p}\right\|^{3}}[
\end{aligned}
$$

where $\mathbf{r}_{i, a / p}$ and $\mathbf{v}_{i, a / p}$ are the apogee (or perigee) position and velocity vectors obtained using Eqs. (4.21) and (4.22).

The partial derivatives of $f, g, \dot{f}$ and $\dot{g}$ in Eqs. (9.19) and (9.20) are:

$$
\begin{gathered}
\frac{\partial f}{\partial \mathbf{x}}=\frac{-2 \chi}{\|\mathbf{r}\|} c_{2} \frac{\partial \chi}{\partial \mathbf{x}} \\
\frac{\partial g}{\partial \mathbf{x}}=1-\frac{3 \chi^{2}}{\sqrt{\mu}} c_{3} \frac{\partial \chi}{\partial \mathbf{x}} \\
\frac{\partial \dot{f}}{\partial \mathbf{x}}=\frac{\sqrt{\mu}}{\|\mathbf{r}\| R}\left(\psi c_{3}-1\right) \frac{\partial \chi}{\partial \mathbf{x}} \\
\frac{\partial \dot{g}}{\partial \mathbf{x}}=\frac{-2 \chi}{R} c_{2} \frac{\partial \chi}{\partial \mathbf{x}}
\end{gathered}
$$

where,

$$
\frac{\partial \chi}{\partial \mathbf{x}}=\sqrt{\mu} \gamma \frac{\partial \Delta t}{\partial \mathbf{x}}
$$

where the vector $\mathbf{x}$ represents:

$$
\mathbf{x}=\left[\begin{array}{l}
\mathbf{r} \\
\mathbf{v}
\end{array}\right]
$$

